Max-Planck-Institut für demografische Forschung

Max Planck Institute for Demographic Research

Doberaner Strasse 114 - D-18057 Rostock · GERMANY

Tel +49 (0) 3812081 - 0; Fax +49 (0) 3812081 - 202;

http://www.demogr.mpg.de

MPIDR WORKING PAPER WP 2001-024

AUGUST 2001

\title{
Life-table representations
}

of family dynamics

in 16 FFS countries

Gunnar Andersson (andersson@ demogr.mpg.de)

Dimiter Philipov (philipov@demogr.mpg.de)

This working paper has been approved for release by: Jan M. Hoem (hoem@ demogr.mpg.de)

Head of the Laboratory of Contemporary European Fertility and Family Dynamics.

(C) Copyright is held by the authors.

Working papers of the Max Planck Institute for Demographic Research receive only limited review. Views or opinions expressed in working papers are attributable to the authors and do not necessarily reflect those of the Institute. 


\title{
Max Planck Institute for Demographic Research, Doberaner Straße 114, D-180 57 Rostock, Germany. \\ E-mail: andersson@demogr.mpg.de, philipov@demogr.mpg.de.
}

GA/DP, 2 August 2001

\section{Life-table representations of family dynamics in 16 FFS countries}

\author{
by \\ Gunnar Andersson \\ and \\ Dimiter Philipov
}

\begin{abstract}
In this paper, we apply a system of description of family-demographic behavior to data derived from a number of Fertility and Family Surveys (FFS) conducted in Europe - and to corresponding survey data from the USA. We use lifetable techniques in order to describe the experiences of men, women, and children in processes related to family formation and family dissolution during the late 1980s early 1990s. Our presentation amounts to a large number of cross-country comparisons of the demographic behavior in Eastern and Western Europe with the US used as a contrast. Our system is described in more detail in Andersson and Philipov (2001) and here we apply it to data from Sweden, Norway, Finland, France, USA, Austria, Germany (East and West Germany separately), Flanders, Italy, Spain, Czech Republic, Hungary, Slovenia, Latvia, Lithuania, and Poland.
\end{abstract}




\section{Introduction}

The purpose of this paper is to provide an extensive number of life tables that give information on various aspects of family demographic behavior in a range of developed countries by describing various aspects of life trajectories of people in these countries. Our tabulations describe different types of family experience from the point of view of men, of women, and of children.

The present study essentially amounts to the application of an existing system of description of demographic behavior, presented in Andersson and Philipov (2001), to data from 16 different countries. In Andersson and Philipov (2001), we initiated the present project by providing detailed information on how our system of life tables is constructed and what our tables describe. We then used data from Hungary and Sweden in order to give an example of what kind of cross-country comparison our system can provide. In the present paper, we give tabulations of the same kind but now apply our system to data from a larger number of countries which participated in the round of Fertility and Family Surveys (FFS) that was conducted in Europe between 1989 and 1997. We use corresponding data from the US National Survey of Family Growth of 1995 in order to contrast patterns in Europe to those prevailing on the other side of the Atlantic.

In the present manuscript, we do not even try to make any comments or reflections on the abundance of information that arises from the tabulations we give. We merely present our results to a broader audience in order to open up for further contemplation. It is our hope that the tabulations we produce will provide a better picture of various aspects of family dynamics in the countries we study and that they can serve as a basis for a number of more specific cross-country comparisons. Evidently, if one choose to focus on just a subset of our tabulations, one can derive detailed information on a certain topic in family demography - as seen from the point of view of men, of women, or of children. In the present manuscript, we provide our tabulations in an Addendum to the paper, which otherwise just gives a short description of the data at hand and a brief overview of the tables we present.

\section{Life-table descriptions}

Our descriptive measures are based on well-known life-table techniques and we have constructed our life tables from the notion of a synthetic cohort, based on the information pertaining to a period immediately before each FFS survey of interest. While a calculation of life tables for a real birth cohort of men, women, or children or a real marriage cohort - is a straightforward matter, it requires that one can follow the cohort of interest up to the highest age limit of the tabulation, for example up to age 40 . We prefer to be able to say something about the demographic behavior during the most recent calendar period. For that purpose, we construct a life table from reported events of a specific kind and exposures to that event during our period of interest, i.e., during the latest period for which we have data, so that we can describe the most recent patterns of family-demographic affairs.

In principle, we present three broad groups of tabulations, all described in more detail in Andersson and Philipov (2001). First, we give a number of measures of various aspects of family formation of men and women in the different countries. These show the cumulative percent of men and women who have experienced a specific demographic event by single-year ages from their $15^{\text {th }}$ to their $40^{\text {th }}$ birthday. Secondly, we present a number of measures of various experiences of men and women in unions, by duration of union. Here we use the union as our unit of 
observation and we pool the information on unions as reported by women and men. We report various types of experiences in different types of unions. For example, we report the cumulative percent of consensual unions who are being dissolved or are being transformed into a marriage by single-year durations up to the $15^{\text {th }}$ or $20^{\text {th }}$ birthday of the union. Thirdly, we present various life-table measures of children's experience of family formation and family dissolution. These measures are calculated from the information given by their mothers' reports of union events and they are presented by single-year ages for the child, from birth to the $15^{\text {th }}$ birthday. We present separate calculations for children born to a lone mother, children born to a cohabiting mother, and children born in marriage so that we can depict the different familytransformation events that can occur in each type of family. Finally, our various lifetable measures are summarized in a few tables and figures that report the fractions of total time that men, women, and children typically spend in various family types during their reproductive ages or during childhood.

The following list of contents gives an overview of the tabulations we present. We provide 45 different sets of life tables. Each set of tables is presented with one sub-set for the various Western European countries of our study, including the US, and a second sub-set for the Eastern European countries that participated in the FFS. Our Tables 1-16 are calculated for men and for women, separately, so in these cases, we also provide separate sub-sets of tabulations for the two sexes.

\section{LIST OF CONTENTS}

Tables 1-16: Single-sex tables of family formation of men and women

\section{Experience of nest-leaving}

1- leaving the parental home

\section{Experience of union formation}

2- first union, as a cohabitation (censoring at marriage)

3- first union, as a marriage (censoring at cohabitation)

4- first union, as a cohabitation (competing-risks model)

5- first union, as a marriage (competing-risks model)

6- first union, as a marriage or a cohabitation (4+5)

7- first marriage

\section{Experience of becoming a parent}

8- first child

Experience of the combination of being a parent and being in different union statuses

9- parent and in a union

10- parent and married

11- parent and not in a union

Experience of different contexts of family-formation/childbearing events

12- marriage during a first union

13- first child during a first union

14- first child during any union

15- first child during any marriage

16- first child born out of a union 
Childless couples: experience of childbearing or separation

17- first child of a childless couple

18- separated before a birth, childless couple

Consensual unions: experience of marriage or separation

19- married before dissolution

20- separated before marriage

21- married (competing-risks model)

22- separated (competing-risks model)

23- no longer in a consensual union $(21+22)$

Couples experience of union disruption

24- separation for unions begun as a cohabitation

25- separation for unions begun as a marriage

26- separation for all unions

27- separation for all marriages

28- separation of parents in union (duration since union/parenthood)

\section{Summary measure}

29- percent of time spent in different family types (men, women)

\section{Tables 30-45: Children's experience of family dynamics}

\section{Family type at birth}

30- percent of births by family type

Experience of family disruption

31- ever out of union (all children)

32- ever out of marriage (all children)

33- out of union (children born in union)

34- out of union (children born in consensual union)

35- out of union (children born in marriage)

Experience of family formation

36- in union (children born to lone mother)

37- in marriage (children born to lone mother)

38- in marriage (children born to cohabiting mother)

39- in marriage (children born to non-married mother)

Competing-risks model for children born in consensual union

40- in marriage

41- out of union

42- no longer in consensual-union family $(40+41)$

Experience of family re-formation

43- again in union (after parental separation)

44- in marriage (after parental separation)

Summary measure

45- percent of time spent in different family types

In our tabulations, we also present a number of mean and percentile values in order to derive some summary information from each life table. We present mean ages of transition for those individuals who experience an event of interest before the upper age limit of the table. We also present the first exact ages where at least 10, 25, 
50, and 75 percent of the study population first experience the event under study, i.e., the ages of the first decile, the first quartile, the median, and the third quartile. We sometimes also use a truncated mean value that gives information on the average amount of time that is spent in a certain family status during the interval we study. Such a mean value also include the experience of individuals who stay in the risk population until the end of the interval.

\section{Data}

Our tabulations are based on raw data gathered at the Fertility and Family Surveys that were conducted in a large number of European countries in 1989-1997. We have used comparable data from the US National Survey of Family Growth in order to derive corresponding tabulations for the USA. The data have been provided to us by the Population Activities Unit in Geneva but for Sweden we have instead used a cleaned national version of the data.

We have calculated life tables for the following countries: Sweden, Norway, Finland, France, USA, Austria, Germany, Flanders ${ }^{1}$, Italy, Spain, Czech Republic, Hungary, Slovenia, Latvia, Lithuania, and Poland. In our calculations of life tables for Germany, we treated the Eastern and Western parts of the country as two separate entities. We have not used existing data from Bulgaria and Portugal since they do not contain union histories of respondents and thus are not suitable for calculations like ours. Existing data from Netherlands are not available to researchers outside that country but in the future we hope instead to be able to add a few other countries to our descriptive analysis ${ }^{2}$.

\section{3a. Cleaning procedures}

Before calculating the various life-table measures we present, we had to perform a considerable amount of data cleaning. As a result, we have removed a number of respondents from the initial study population with incomplete information or illogical sequences of events. The raw data for Austria and Germany, just to mention one example, contained a large number of observations with no dates of union formation and union dissolution for respondents who reported that they had experienced at least one union, so we had to exclude them from our study population. Table 1 contains the total number of male and female respondents, mothers, and children of female respondents that, for each country, remain in our data set after our cleaning. We do not include any children as reported by fathers since children more often not co-reside with a father than with a mother and larger segments of children's lives thus are missing in their reports.

When we found a case with missing information, so that we could not use it in our computations of life tables, we first tried to impute the piece of information that was missing, or we had to exclude the individual from our study population. Most of our cleaning procedures refer to cases with lacking dates (year, month, or year and month) of reported events. In cases where we know the year of a reported event but not its month, we can often impute the value of the latter. This is possible when there

\footnotetext{
${ }^{1}$ The Belgian FFS only covers the Flemish-speaking parts of the country.

${ }^{2}$ We have not (yet) made calculations for Switzerland, Estonia, or overseas New Zealand and Canada. In the future, we hope to be able to apply our system of description also to data from these countries and to similar survey data from Great Britain.
} 
is no other family-demographic event reported for the same year or when we know if any other event has occurred before or after the incompletely reported event (for example, if we know a date that refers to a preceding or a subsequent union). In the case of remaining inconsistency in reports of childbearing, union formation, or union dissolution, we exclude the erroneous case from all our life-table calculations even if it could perhaps be used for the estimation of some sub-set of life tables. However, in the case of missing information on just the date of leaving the parental home, we drop only the information that pertains to the nest-leaving so that we can use the individual for the calculation of all remaining life tables.

Table 1: Size of study population, by country

\begin{tabular}{lrrrrr} 
& men & women & mothers & children \\
\cline { 5 - 5 } Austria & 1428 & 4260 & 3217 & 6485 \\
Flanders & 2104 & 3143 & 1911 & 3602 \\
Czech Rep. & -- & 1719 & 1222 & 2331 \\
Finland & 1592 & 4040 & 2895 & 6043 \\
France & 1915 & 2930 & 2194 & 4527 \\
E Germany & 1875 & 2810 & 2025 & 3437 \\
W Germany & 1863 & 2743 & 1223 & 2113 \\
Hungary & 1899 & 3498 & 2622 & 4908 \\
Italy & 1175 & 4745 & 2858 & 5410 \\
Latvia & 1338 & 2622 & 2080 & 3787 \\
Lithuania & 1948 & 2924 & 2113 & 3742 \\
Norway & 1515 & 3969 & 2367 & 4523 \\
Poland & -- & 4165 & 3184 & 6752 \\
Slovenia & 1716 & 2761 & 2116 & 3953 \\
Spain & 1951 & 3981 & 2450 & 4991 \\
Sweden & 1495 & 2986 & 2247 & 4638 \\
USA & -- & 10510 & 6609 & 14357
\end{tabular}

In Table 2, we report the number of excluded individuals, by type of error that we first found for a respondent, by country. In the section below, we also report the steps of the cleaning procedure that produced these exclusions.

1. If the month of birth of a respondent is missing, we impute the value of that month randomly. A missing month of interview is imputed to a late month of the period when the other interviews were performed.

2. Erroneous information on union formation: excluded cases are reported in Table 2 under the heading form. (for formation). We drop an individual if the number of unions is unknown or the year of a union formation is unknown. Examples of this kind occurred, for example, when we found individuals with no date of union formation but information that elsewhere said that the respondent indeed had entered a union (mainly in the data for Germany and Austria). In some countries, the number of reported unions for an individual sometimes seemed to be too high because of an incorrect summation of cohabitation and marriage pertaining to the same union (common in the data for Lithuania and Germany). In this situation, we keep the individual and use the number of unions as appearing in the sequence of reported events of union formation and union dissolution. We drop an individual if the month of a union formation is unknown and a birth is reported in the same year, since we need to know in what order such events have occurred. However, if there is no other event reported in the same year, we impute the month to a 
position in the middle of the year (common in the data for Austria, for dates of first cohabitation). If the union is dissolved in the same year as it is being formed, we impute a missing month of union formation to a lower value than that of the dissolution. If a previous union is dissolved in the same year, we impute the month to a higher value than that of the dissolution. If a marriage occurs in the same year and we have no month of union formation, we assume that it begins as a direct marriage, i.e., at marriage formation.

3. Erroneous information on union dissolution: excluded cases are reported in Table 2 under the heading disr. (for disruption). We drop an individual if the year of a reported dissolution is unknown or if the date of dissolution is lower than the date of the formation of the same union. If a date of union dissolution is higher than that of the formation of a subsequent union, we instead change the date to be equal to the latter one. Missing months of union dissolution are handled in the same way as we handle missing months of union formation.

4. Erroneous information on childbearing: excluded cases are reported in Table 2 under the heading childb. We drop an individual if the year and/or the month of the birth of a child (or the adoption of an adopted child) is unknown.

In addition, we exclude all male respondents from the surveys of Poland and the Czech Republic since they are all partners to the female respondents there. It makes no sense to study family-formation events of a population where we have no information on individuals who have not experienced the events of interest. (There are no men in the study population for the US either but this is just because no men were interviewed in the survey that was launched in the USA.)

Table 2 first gives the initial number of respondents from each country (for Poland and the Czech Republic, these numbers only cover female respondents). The following three columns give the number of exclusions from errors of the types we reported above. A column marked $\underline{\text { misc }}$. (for miscellaneous) reports additional omissions of individuals due to various other types of errors. We proceed to report the total number of deleted cases by country, and the percent of deleted individuals of the initial number of respondents. The last columns give the final number of respondents in our analysis, also given for $\underline{\text { men }}$ and women separately.

Table 2: Number of respondents and exclusions of respondents from our sample

\begin{tabular}{lrrrrrrrrrrrrr} 
Country & initial & & form. & \multicolumn{1}{c}{ disr. } & childb. & & misc. & deleted & percent & & final & & men women \\
Austria & 6120 & 243 & 32 & 155 & 2 & 432 & 7.1 & 5688 & 1428 & 4260 \\
Flanders & 5433 & 11 & 17 & 153 & 5 & 186 & 3.4 & 5247 & 2104 & 3143 \\
Czech Rep. & 1735 & 3 & 8 & 0 & 5 & 16 & 0.9 & 1719 & -- & 1719 \\
Finland & 5825 & 12 & 25 & 139 & 17 & 193 & 3.3 & 5632 & 1592 & 4040 \\
France & 4885 & 0 & 1 & 37 & 2 & 40 & 0.8 & 4845 & 1915 & 2930 \\
E Germany & 4976 & 129 & 62 & 94 & 6 & 291 & 5.8 & 4685 & 1875 & 2810 \\
W Germany & 5036 & 292 & 56 & 79 & 3 & 430 & 8.5 & 4606 & 1863 & 2743 \\
Hungary & 5487 & 20 & 35 & 34 & 1 & 90 & 1.6 & 5397 & 1899 & 3498 \\
Italy & 6030 & 44 & 24 & 23 & 19 & 110 & 1.8 & 5920 & 1175 & 4745 \\
Latvia & 4200 & 5 & 8 & 218 & 9 & 240 & 5.7 & 3960 & 1338 & 2622 \\
Lithuania & 5000 & 70 & 57 & 0 & 1 & 128 & 2.6 & 4872 & 1948 & 2924 \\
Norway & 5562 & 57 & 19 & 1 & 1 & 78 & 1.4 & 5484 & 1515 & 3969 \\
Poland & 4211 & 2 & 0 & 44 & 0 & 46 & 1.1 & 4165 & -- & 4165 \\
Slovenia & 4559 & 23 & 16 & 38 & 5 & 82 & 1.8 & 4477 & 1716 & 2761 \\
Spain & 6013 & 15 & 11 & 41 & 14 & 81 & 1.3 & 5932 & 1951 & 3981 \\
Sweden & 4984 & -- & -- & -- & 503 & 503 & 10.1 & 4481 & 1495 & 2986 \\
USA & 10847 & 62 & 234 & 40 & 1 & 337 & 3.1 & 10510 & -- & 10510
\end{tabular}


For our calculations of life tables of the process of leaving the parental home, we had to make some additional exclusions. These were made if we had no information on the year of leaving the parental home but knew that a respondent had left home. As usual, a missing month was imputed to a value at the middle of the year.

In our analyses of children's family dynamics, we also had to make some additional exclusions that are not reported in Table 2. These refer to children who no longer lived with their mother but had no date given for their departure from the mother. The situation mainly appears in the data for Norway where dates of children's departures are not given. For Lithuania and Poland, we have no information at all on the residential situation of children so we do not know if a child still lives with the mother at the time of interview. For these two countries, we simply assume that each reported child continue to live with its mother until the $15^{\text {th }}$ birthday (which is the upper age limit of our life tables on the demographic experience of children). This is not a problematic assumption since the data we have for the other countries reveal that practically all children actually live with their mother until that age. Finally, in the data set for France, we cannot distinguish between the situation when a child dies from that when it stops living with its mother. We keep these children in our analysis but censor our observation at any event of that kind.

For Sweden, we have used a data set that has already been cleaned in more detail at Statistics Sweden and at the Stockholm University Demography Unit. In this case, we have also excluded all respondents who immigrated to Sweden when being older than 15 years. In Table 2, these and other omissions show up under the heading

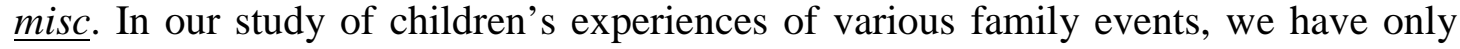
included children who were born in Sweden. In the case of migration during the age segments that we want to study, we expect to find some interdependence between migration and family formation and we do not want to deal with such issues here. For other countries of the FFS "Standard Recode Files", we have no information on country of birth of survey respondents. This means that possible immigrants are included in our study and that we cannot distinguish between demographic events that occurred in the country of origin and in the country of destination, since we have no information about actual dates of immigration. This is a less desirable feature of our data, which we unfortunately cannot deal with further.

\section{3b. Synthetic cohorts}

In our calculations, we have subsequently used the parts of the data that refer to the six-year period immediately before each interview. Initially we aimed at estimating measures for the five-year period before each survey but in order to cover the demographic histories of men and women from ages as low as 15 years, we needed to go six years back in time. Many countries do not include enough young respondents in their surveys. For Finland we had to use an even longer period for the construction of our synthetic cohort since the male respondents of that country all were 25 years or older. The same holds for Norway and Sweden where the youngest male respondents were 28 and 29 years old, respectively. The samples from Norway and Sweden differ from those of other countries also in the respect that they cover respondents from just a selected number of birth cohorts of men and women. In principle, this makes these data sets more suitable for an analysis of the behavior of real birth cohorts of men and women but we apply our idea of a synthetic cohort also to these countries data since we aim at comparability over our whole range of countries. However, in any comparison between countries, we need to be aware that our life tables for Sweden, Norway, and Finland are based on longer calendar periods than those of the other 
countries and that important changes in behavior may have occurred during such extended study periods. (When we study unions and children of respondents from these countries, we can use a shorter period for our synthetic-cohort measures, better corresponding to the six-year period of the other countries.) Finally, for East Germany, we use the six-year period that refers to the time immediately before the political turnaround in November 1989 as our study period, which thus describes the situation in the last years of the former $\mathrm{GDR}^{3}$. In Table 3 , we report the calendar periods that we use in order to construct our synthetic cohorts for each country in our study.

Table 3: Study period of life tables of men, of women, of unions, and of children

\begin{tabular}{lrrr} 
Country & women/men & $\underline{\text { unions }}$ & children \\
\cline { 2 - 4 } Austria & $1990-1996$ & $1990-1996$ & $1990-1996$ \\
Flanders & $1985-1992$ & $1985-1992$ & $1985-1992$ \\
Czech Rep. & $1992-1997$ & $1992-1997$ & $1992-1997$ \\
Finland & $1979 / 83-1989 / 92$ & $1983-1992$ & $1983-1989$ \\
France & $1988-1994$ & $1988-1994$ & $1988-1994$ \\
E Germany & $1984-1989$ & $1984-1989$ & $1984-1989$ \\
W Germany & $1986-1992$ & $1986-1992$ & $1986-1992$ \\
Hungary & $1988-1993$ & $1988-1993$ & $1988-1993$ \\
Italy & $1990-1995$ & $1990-1995$ & $1990-1995$ \\
Latvia & $1989-1995$ & $1989-1995$ & $1989-1995$ \\
Lithuania & $1989-1995$ & $1989-1995$ & $1989-1995$ \\
Norway & $1974-1989$ & $1983-1989$ & $1983-1989$ \\
Poland & $1986-1991$ & $1986-1991$ & $1986-1991$ \\
Slovenia & $1989-1995$ & $1989-1995$ & $1989-1995$ \\
Spain & $1989-1995$ & $1989-1995$ & $1989-1995$ \\
Sweden & $1978-1993$ & $1985-1993$ & $1987-1993$ \\
USA & $1989-1995$ & $1989-1995$ & $1989-1995$
\end{tabular}

We calculate our life tables from estimated probabilities of exit from each study population separately, caused by a specific event under study, and probabilities of surviving in the original state. If the number of persons under risk of experiencing the specific event decreases to less than 15 individuals (at some higher duration or age segment), we freeze our life-table estimates at that stage. We are unable to present any further figures for durations above that time horizon. This can occur, for example, when the number of unions of a specific type, like consensual unions in a Southern European country, is too small for an extended investigation of their destinies. It can also occur when practically the whole study population experience a particular event already at a relatively early stage, like the process of leaving the parental home in the Nordic countries.

We have applied weights in our calculations of life-table estimates for France and the US since the sampling procedures in these countries were performed in a way that were dependent on the outcomes we want to study. As a final remark, we just want to point to the need for further data validation in this project - as in most other projects that use standard files of FFS data. Despite our cleaning efforts, we have not

\footnotetext{
${ }^{3}$ The oldest respondents of the East German sample were 36 years old in 1989. This has consequences for the calculation of several of our life tables since we have no observations at the highest age interval of our Tables 1-16. In Table 29, we cannot calculate a summary measure of the time that men and women in East Germany typically spent in different family types at ages 15-39.
} 
been able to go through all details at all corners of our data sets. We have also not been able to make any deeper investigation of, for example, differences in sampling procedures between countries. One important issue is to what degree immigrants tend to show up in the various data sets we have used. In our case, we have just used the data provided to us but have at least removed a number of evident cases of inconsistency in it.

\section{Acknowledgments}

We are grateful to Jan M. Hoem for valuable advice during the work with this project and to discussions with him, Larry Bumpass, Ron Lesthaeghe, and Kathleen Kiernan during a working meeting on this project in Brussels on 27 May 2000. Many of the life-table measures we have used in our presentation were first suggested to us by Larry Bumpass. We thank the Advisory Group of the European Fertility and Family Surveys (FFS) program of comparative research for its permission, granted under identification number 75, to use the original FFS data for this study. In addition, we are grateful to the Stockholm University Demography Unit for its permission to use their cleaned version of the Swedish FFS. Finally, we thank Friederike Seichter for practical assistance in putting all our tables together.

\section{Reference}

Andersson, G., and Philipov, D., 2001. Life-table representations of family dynamics in Sweden and Hungary: initiation of a project of descriptions of demographic behavior. MPIDR Working Paper, WP 2001-010. Max Planck Institute for Demographic Research, Rostock.

\section{Addendum: Tables 1-45.}

We derive the following number of life tables in our presentation: Tables 1-16 and 29, for men and women in $17^{4}$ countries: 578 tables, Tables 17-28, for unions in 17 countries: 204 tables,

Tables 30-45, for children in 17 countries:

Total number of life tables: 272 tables, 1054.

\footnotetext{
${ }^{4}$ We have 17 countries when East and West Germany are counted separately.
} 
Table 1: Cumulative percent ever leaving the parental home / Men

\begin{tabular}{|c|c|c|c|c|c|}
\hline Age & $\begin{array}{l}\text { Sweden } \\
(1978-93)\end{array}$ & $\begin{array}{l}\text { Norway } \\
(1974-89)\end{array}$ & $\begin{array}{l}\text { Finland } \\
(1983-92)\end{array}$ & $\begin{array}{l}\text { France } \\
(1988-94)\end{array}$ & $\begin{array}{l}\text { USA } \\
\text { (NA) }\end{array}$ \\
\hline 16 & 4 & 3 & 2 & 3 & \\
\hline 18 & 19 & 11 & 9 & 8 & \\
\hline 20 & 46 & 32 & 26 & 22 & \\
\hline 22 & 78 & 56 & 51 & 37 & \\
\hline 24 & 91 & 76 & 69 & 61 & \\
\hline 25 & 94 & 80 & 76 & 68 & \\
\hline 26 & 96 & 85 & 80 & 76 & \\
\hline 28 & 98 & 90 & 85 & 83 & \\
\hline 30 & 98 & 93 & 87 & 86 & \\
\hline 35 & -- & 96 & 88 & 91 & \\
\hline 40 & -- & 98 & 89 & -- & \\
\hline $\begin{array}{l}\text { mean age: } \\
\text { (at transition) }\end{array}$ & 20 & 22 & 22 & 23 & \\
\hline 1st decile at age: & 17 & 18 & 19 & 19 & \\
\hline 1st quartile at: & 19 & 20 & 20 & 21 & \\
\hline median at age: & 21 & 22 & 22 & 24 & \\
\hline 3rd quartile at: & 22 & 24 & 25 & 26 & \\
\hline age & $\begin{array}{c}\text { Austria } \\
(1990-96)\end{array}$ & $\begin{array}{c}\text { West } \\
\text { Germany } \\
(1986-92)\end{array}$ & $\begin{array}{l}\text { Flanders } \\
(1985-92)\end{array}$ & $\begin{array}{c}\text { Italy } \\
(1990-95)\end{array}$ & $\begin{array}{c}\text { Spain } \\
(1989-95)\end{array}$ \\
\hline 16 & 3 & 2 & 1 & 1 & 1 \\
\hline 18 & 7 & 4 & 2 & 3 & 2 \\
\hline 20 & 24 & 19 & 8 & 7 & 6 \\
\hline 22 & 40 & 46 & 21 & 10 & 12 \\
\hline 24 & 58 & 66 & 46 & 16 & 23 \\
\hline 25 & 63 & 75 & 59 & 20 & 31 \\
\hline 26 & 67 & 79 & 68 & 24 & 39 \\
\hline 28 & 76 & 86 & 79 & 38 & 55 \\
\hline 30 & 82 & 90 & 87 & 49 & 64 \\
\hline 35 & 88 & 93 & 91 & 72 & 80 \\
\hline 40 & 91 & -- & -- & 75 & 83 \\
\hline $\begin{array}{l}\text { mean age: } \\
\text { (at transition) }\end{array}$ & 23 & 23 & 24 & 28 & 26 \\
\hline 1st decile at age: & 19 & 20 & 21 & 22 & 22 \\
\hline 1st quartile at: & 21 & 21 & 23 & 27 & 25 \\
\hline median at age: & 23 & 23 & 25 & 31 & 28 \\
\hline 3rd quartile at: & 28 & 25 & 28 & 40 & 33 \\
\hline
\end{tabular}


Table 1: Cumulative percent ever leaving the parental home / Men

age

16

18

20

22

24

25

26

28

30

35

40

mean age:

(at transition)

1st decile at age:

1st quartile at:

median at age:

3 rd quartile at:

age

16

18

20

22

24

25

26

28

30

35

40

mean age:

(at transition)

1st decile at age:

1st quartile at:

median at age:

3 rd quartile at:
GDR Hungary Czech R Slovenia Latvia $\begin{array}{llll}(1984-89) & (1988-93) \quad(N A) \quad(1989-95) & (1989-95)\end{array}$

1

62

$21 \quad 7$

$38 \quad 19$

$58 \quad 35$

$70 \quad 43$

$78 \quad 49$

$86 \quad 58$

$90 \quad 65$

$93 \quad 74$

$--\quad 75$

$23 \quad 25$

$25-23$

$\begin{array}{llll}19 & 21 & 19 & 20\end{array}$

$21 \quad 23$

$24 \quad 27$

$26 \quad 39$

$21 \quad 22$

$24 \quad 27$

30 --

31

44

53

59

63

66

70

71

23

19

22

25 
Table 1: Cumulative percent ever leaving the parental home / Women

\begin{tabular}{|c|c|c|c|c|c|}
\hline age & $\begin{array}{l}\text { Sweden } \\
(1978-93)\end{array}$ & $\begin{array}{l}\text { Norway } \\
(1974-89)\end{array}$ & $\begin{array}{l}\text { Finland } \\
(1979-89)\end{array}$ & $\begin{array}{l}\text { France } \\
(1988-94)\end{array}$ & $\begin{array}{c}\text { USA } \\
(1989-95)\end{array}$ \\
\hline 16 & 6 & 4 & 4 & 1 & 2 \\
\hline 18 & 29 & 19 & 18 & 9 & 17 \\
\hline 20 & 73 & 52 & 50 & 36 & 66 \\
\hline 22 & 93 & 77 & 78 & 60 & 81 \\
\hline 24 & 98 & 88 & 90 & 78 & 88 \\
\hline 25 & 99 & 92 & 93 & 84 & 91 \\
\hline 26 & 99 & 94 & 95 & 88 & 92 \\
\hline 28 & -- & 97 & 97 & 92 & 95 \\
\hline 30 & -- & 98 & 98 & 94 & 96 \\
\hline 35 & -- & 99 & 99 & -- & 97 \\
\hline 40 & -- & -- & 99 & -- & 97 \\
\hline $\begin{array}{l}\text { mean age: } \\
\text { (at transition) }\end{array}$ & 19 & 20 & 20 & 21 & 20 \\
\hline 1st decile at age: & 17 & 17 & 17 & 19 & 18 \\
\hline 1st quartile at: & 18 & 19 & 19 & 20 & 19 \\
\hline median at age: & 19 & 20 & 20 & 22 & 19 \\
\hline 3rd quartile at: & 21 & 22 & 22 & 24 & 21 \\
\hline age & $\begin{array}{c}\text { Austria } \\
(1990-96)\end{array}$ & $\begin{array}{c}\text { West } \\
\text { Germany } \\
(1986-92)\end{array}$ & $\begin{array}{c}\text { Flanders } \\
(1985-92)\end{array}$ & $\begin{array}{c}\text { Italy } \\
(1990-95)\end{array}$ & $\begin{array}{c}\text { Spain } \\
(1989-95)\end{array}$ \\
\hline 16 & 3 & 1 & 0 & 0 & 1 \\
\hline 18 & 14 & 7 & 4 & 2 & 4 \\
\hline 20 & 40 & 34 & 24 & 8 & 13 \\
\hline 22 & 60 & 63 & 48 & 17 & 23 \\
\hline 24 & 74 & 82 & 73 & 29 & 37 \\
\hline 25 & 80 & 87 & 82 & 36 & 47 \\
\hline 26 & 85 & 90 & 88 & 44 & 54 \\
\hline 28 & 90 & 94 & 95 & 55 & 70 \\
\hline 30 & 93 & 96 & 96 & 63 & 77 \\
\hline 35 & 96 & 98 & 97 & 77 & 85 \\
\hline 40 & 97 & -- & -- & 80 & 87 \\
\hline $\begin{array}{l}\text { mean age: } \\
\text { (at transition) }\end{array}$ & 22 & 21 & 22 & 26 & 25 \\
\hline 1st decile at age: & 18 & 19 & 19 & 21 & 20 \\
\hline 1st quartile at: & 19 & 20 & 21 & 24 & 23 \\
\hline median at age: & 21 & 21 & 23 & 27 & 26 \\
\hline 3rd quartile at: & 25 & 23 & 25 & 34 & 30 \\
\hline
\end{tabular}


Table 1: Cumulative percent ever leaving the parental home / Women

age

16

18

20

22

24

25

26

28

30

35

40

mean age:

(at transition)

1st decile at age:

1st quartile at:

median at age:

3 rd quartile at:

age

16

18

20

22

24

25

26

28

30

35

40

mean age:

(at transition)

1st decile at age:

1st quartile at:

median at age:

3rd quartile at:
GDR Hungary Czech R Slovenia Latvia (1984-89) (1988-93) (1992-97) (1989-95) (1989-95)

1

$34 \quad 25$

$63 \quad 46$

$79 \quad 63$

$84 \quad 69$

$88 \quad 72$

$92 \quad 77$

$93 \quad 80$

$95 \quad 83$

$--\quad 84$

$21 \quad 22$

22

$\begin{array}{lllll}19 & 19 & 19 & 19 & 19 \\ 20 & 20 & 20 & 21 & 20 \\ 22 & 23 & 23 & 23 & 25 \\ 24 & 27 & 27 & 27 & --\end{array}$

Lithuania Poland

(1989-95) (1986-91)

$\begin{array}{cc}2 & 1 \\ 9 & 4 \\ 34 & 21 \\ 48 & 41 \\ 57 & 54 \\ 60 & 60 \\ 63 & 64 \\ 68 & 70 \\ 71 & 73 \\ 73 & 79 \\ 73 & 82\end{array}$

21

23

$19 \quad 19$

$20 \quad 21$

$23 \quad 24$

-- 32 
Table 2: Cumulative percent ever starting a first union as a cohabitation, single-decrement life-table method with censoring at direct marriage / Men

$\begin{array}{cccccc}\text { age } & \text { Sweden } & \text { Norway } & \text { Finland } & \text { France } & \text { USA } \\ (1978-93) & (1974-89) & (1983-92) & (1988-94) & \text { (NA) }\end{array}$

$\begin{array}{lcccc}16 & 0 & 0 & 0 & 0 \\ 18 & 3 & 1 & 3 & 3 \\ 20 & 16 & 8 & 9 & 10 \\ 22 & 40 & 25 & 23 & 21 \\ 24 & 60 & 46 & 44 & 39 \\ 25 & 67 & 55 & 51 & 48 \\ 26 & 72 & 61 & 59 & 56 \\ 28 & 80 & 73 & 68 & 67 \\ 30 & 84 & 76 & 74 & 72 \\ 35 & 90 & 84 & 80 & 80 \\ 40 & 92 & 87 & 83 & 83 \\ & & & & \\ \text { mean age: } & 23 & 25 & 25 & 25 \\ \text { (at transition) } & & & & \\ & & & & \\ \text { 1st decile at age: } & 20 & 21 & 21 & 20 \\ \text { 1st quartile at: } & 21 & 22 & 23 & 23 \\ \text { median at age: } & 23 & 25 & 25 & 26 \\ \text { 3rd quartile at: } & 27 & 30 & 31 & 31\end{array}$

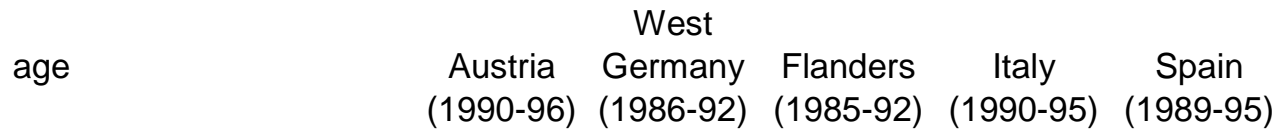

$\begin{array}{lccccc}16 & 0 & 0 & 0 & 0 & 0 \\ 18 & 2 & 0 & 1 & 0 & 0 \\ 20 & 12 & 4 & 3 & 1 & 2 \\ 22 & 23 & 10 & 7 & 1 & 4 \\ 24 & 36 & 19 & 17 & 3 & 7 \\ 25 & 41 & 24 & 24 & 4 & 10 \\ 26 & 47 & 27 & 26 & 5 & 13 \\ 28 & 57 & 34 & 38 & 5 & 19 \\ 30 & 67 & 40 & 48 & 8 & 20 \\ 35 & 77 & 48 & 57 & 20 & 28 \\ 40 & -- & -- & -- & -- & 28 \\ & & & & & \\ \text { mean age: } & 25 & 26 & 27 & 32 & 27 \\ \text { (at transition) } & & & & & \\ & & & & & \\ \text { 1st decile at age: } & 20 & 22 & 23 & 32 & 25 \\ \text { 1st quartile at: } & 23 & 26 & 26 & 39 & 33 \\ \text { median at age: } & 27 & -- & 31 & -- & - \\ \text { 3rd quartile at: } & 35 & -- & -- & -- & --\end{array}$


Table 2: Cumulative percent ever starting a first union as a cohabitation, single-decrement life-table method with censoring at direct marriage / Men

\begin{tabular}{|c|c|c|c|c|c|}
\hline age & $\begin{array}{c}\text { GDR } \\
(1984-89)\end{array}$ & $\begin{array}{l}\text { Hungary } \\
(1988-93)\end{array}$ & $\begin{array}{c}\text { Czech R } \\
\text { (NA) }\end{array}$ & $\begin{array}{c}\text { Slovenia } \\
(1989-95)\end{array}$ & $\begin{array}{r}\text { Latvia } \\
(1989-95\end{array}$ \\
\hline 16 & 0 & 0 & & 0 & 0 \\
\hline 18 & 1 & 1 & & 0 & 3 \\
\hline 20 & 7 & 5 & & 3 & 10 \\
\hline 22 & 17 & 12 & & 16 & 24 \\
\hline 24 & 34 & 20 & & 30 & 38 \\
\hline 25 & 44 & 25 & & 37 & 46 \\
\hline 26 & 52 & 30 & & 46 & 47 \\
\hline 28 & 63 & 37 & & 60 & 58 \\
\hline 30 & 70 & 39 & & 67 & 58 \\
\hline 35 & 77 & 48 & & 82 & -- \\
\hline 40 & -- & 53 & & -- & -- \\
\hline $\begin{array}{l}\text { mean age: } \\
\text { (at transition) }\end{array}$ & 25 & 26 & & 26 & 24 \\
\hline 1st decile at age: & 21 & 22 & & 21 & 20 \\
\hline 1st quartile at: & 23 & 25 & & 24 & 23 \\
\hline median at age: & 26 & 37 & & 27 & 27 \\
\hline 3rd quartile at: & 34 & -- & & 31 & -- \\
\hline
\end{tabular}

$\begin{array}{cc}\text { Lithuania } & \text { Poland } \\ (1989-95) & \text { (NA) }\end{array}$

$16-0$

$18-1$

$20 \quad 4$

$22 \quad 12$

$24-21$

$25 \quad 23$

$26 \quad 27$

$28-35$

$30 \quad 37$

$35 \quad 46$

40 - -

mean age: $\quad 25$

(at transition)

1st decile at age: $\quad 22$

1st quartile at: $\quad 26$

median at age: $\quad$--

3rd quartile at: 
Table 2: Cumulative percent ever starting a first union as a cohabitation, single-decrement life-table method with censoring at direct marriage / Women

\begin{tabular}{|c|c|c|c|c|c|}
\hline age & $\begin{array}{l}\text { Sweden } \\
(1978-93)\end{array}$ & $\begin{array}{c}\text { Norway } \\
(1974-89)\end{array}$ & $\begin{array}{l}\text { Finland } \\
(1979-89)\end{array}$ & $\begin{array}{c}\text { France } \\
(1988-94)\end{array}$ & $\begin{array}{c}\text { USA } \\
(1989-95)\end{array}$ \\
\hline 16 & 2 & 1 & 1 & 0 & 1 \\
\hline 18 & 12 & 8 & 7 & 4 & 8 \\
\hline 20 & 38 & 27 & 25 & 20 & 23 \\
\hline 22 & 62 & 49 & 50 & 39 & 39 \\
\hline 24 & 76 & 64 & 67 & 58 & 50 \\
\hline 25 & 81 & 70 & 74 & 66 & 55 \\
\hline 26 & 84 & 75 & 77 & 71 & 58 \\
\hline 28 & 88 & 81 & 83 & 77 & 65 \\
\hline 30 & 91 & 85 & 86 & 80 & 68 \\
\hline 35 & 94 & 88 & 89 & 85 & 74 \\
\hline 40 & 95 & 89 & 91 & 87 & 76 \\
\hline $\begin{array}{l}\text { mean age: } \\
\text { (at transition) }\end{array}$ & 22 & 22 & 22 & 23 & 23 \\
\hline 1st decile at age: & 18 & 19 & 19 & 19 & 19 \\
\hline 1st quartile at: & 20 & 20 & 20 & 21 & 21 \\
\hline median at age: & 21 & 23 & 22 & 23 & 24 \\
\hline 3rd quartile at: & 24 & 26 & 26 & 27 & 37 \\
\hline age & $\begin{array}{c}\text { Austria } \\
(1990-96)\end{array}$ & $\begin{array}{c}\text { West } \\
\text { Germany } \\
(1986-92)\end{array}$ & $\begin{array}{c}\text { Flanders } \\
(1985-92)\end{array}$ & $\begin{array}{c}\text { Italy } \\
(1990-95)\end{array}$ & $\begin{array}{c}\text { Spain } \\
(1989-95)\end{array}$ \\
\hline
\end{tabular}

$\begin{array}{lccccc}16 & 1 & 0 & 0 & 0 & 0 \\ 18 & 7 & 2 & 1 & 1 & 2 \\ 20 & 22 & 11 & 6 & 3 & 5 \\ 22 & 42 & 21 & 15 & 4 & 7 \\ 24 & 57 & 34 & 24 & 6 & 11 \\ 25 & 62 & 39 & 31 & 6 & 14 \\ 26 & 67 & 43 & 37 & 7 & 18 \\ 28 & 76 & 53 & 47 & 10 & 24 \\ 30 & 83 & 59 & 51 & 13 & 29 \\ 35 & 88 & 64 & 61 & 24 & 35 \\ 40 & 90 & -- & -- & 26 & 36 \\ & & & & & \\ \text { mean age: } & 23 & 24 & 25 & 28 & 26 \\ \text { (at transition) } & & & & & \\ & & & & & \\ \text { 1st decile at age: } & 19 & 20 & 21 & 28 & 24 \\ \text { 1st quartile at: } & 21 & 23 & 25 & 36 & 29 \\ \text { median at age: } & 24 & 28 & 30 & -- & -- \\ \text { 3rd quartile at: } & 28 & -- & -- & -- & -\end{array}$


Table 2: Cumulative percent ever starting a first union as a cohabitation, single-decrement life-table method with censoring at direct marriage / Women

$\begin{array}{lccccc}\text { age } & \begin{array}{c}\text { GDR } \\ (1984-89)\end{array} & \begin{array}{c}\text { Hungary } \\ (1988-93)\end{array} & \begin{array}{c}\text { Czech R } \\ (1992-97)\end{array} & \begin{array}{c}\text { Slovenia } \\ (1989-95)\end{array} & \begin{array}{c}\text { Latvia } \\ (1989-95)\end{array} \\ 16 & & & & & \\ 18 & 0 & 1 & 1 & 1 & 1 \\ 20 & 2 & 6 & 4 & 5 & 12 \\ 22 & 17 & 14 & 24 & 18 & 28 \\ 24 & 35 & 24 & 36 & 32 & 41 \\ 25 & 49 & 34 & 49 & 48 & 52 \\ 26 & 53 & 38 & 55 & 56 & 57 \\ 28 & 59 & 42 & 62 & 61 & 65 \\ 30 & 67 & 45 & 67 & 73 & 72 \\ 35 & 71 & 51 & 74 & 80 & 76 \\ 40 & 78 & 55 & -- & 83 & 80 \\ & -- & 62 & -- & -- & 81 \\ \text { mean age: } & & & & & \\ \text { (at transition) } & 23 & 25 & 22 & 24 & 23 \\ & & & & & \\ \text { 1st decile at age: } & 20 & 20 & 19 & 19 & 18 \\ \text { 1st quartile at: } & 21 & 23 & 21 & 21 & 20 \\ \text { median at age: } & 25 & 30 & 25 & 25 & 24 \\ \text { 3rd quartile at: } & 32 & -- & -- & 29 & 30\end{array}$

age

16

18

20

22

24

25

26

28

30

35

40

mean age:

(at transition)

1st decile at age:

1st quartile at:

median at age:

3rd quartile at:
Lithuania Poland

(1989-95) (1986-91)

$\begin{array}{cc}0 & 0 \\ 3 & 0 \\ 10 & 1 \\ 19 & 2 \\ 23 & 4 \\ 24 & 5 \\ 28 & 5 \\ 33 & 8 \\ 35 & 9 \\ 41 & 11 \\ 45 & 12\end{array}$

$25 \quad 27$

$20 \quad 32$

26 -- 
Table 3: Cumulative percent ever starting a first union as a marriage,

single-decrement life-table method with censoring at entry into cohabitation / Men

$\begin{array}{lcccc}\text { age } & \begin{array}{c}\text { Sweden } \\ (1978-93)\end{array} & \begin{array}{c}\text { Norway } \\ (1974-89)\end{array} & \begin{array}{c}\text { Finland } \\ (1983-92)\end{array} & \begin{array}{c}\text { France } \\ (1988-94)\end{array} \\ 16 & 0 & 0 & 0 & 0 \\ 18 & 0 & 0 & 0 & 1 \\ 20 & 0 & 2 & 1 & 1 \\ 22 & 1 & 8 & 3 & 5 \\ 24 & 2 & 15 & 6 & 9 \\ 25 & 3 & 19 & 7 & 11 \\ 26 & 4 & 23 & 9 & 15 \\ 28 & 7 & 28 & 12 & 19 \\ 30 & 8 & 42 & 16 & 20 \\ 35 & 14 & 57 & 21 & 23 \\ 40 & 18 & 61 & 28 & 27 \\ & & & & \\ \text { mean age: } & 30 & 28 & 29 & 27 \\ \text { (at transition) } & & & & \\ & & & & \\ \text { 1st decile at age: } & 32 & 23 & 27 & 25 \\ \text { 1st quartile at: } & -- & 27 & 37 & 36 \\ \text { median at age: } & -- & 32 & -- & -- \\ \text { 3rd quartile at: } & -- & -- & -- & --\end{array}$

age

16

18

20

22

24

25

26

28

30

35

40

mean age:

(at transition)

1st decile at age:

1st quartile at:

median at age:

3rd quartile at:
West

Austria Germany Flanders Italy Spain

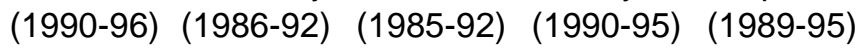

$\begin{array}{ccccc}0 & 0 & 0 & 0 & 0 \\ 0 & 0 & 0 & 0 & 0 \\ 2 & 1 & 0 & 0 & 2 \\ 4 & 3 & 6 & 1 & 5 \\ 9 & 5 & 24 & 6 & 13 \\ 13 & 6 & 34 & 9 & 19 \\ 16 & 9 & 45 & 13 & 25 \\ 19 & 13 & 53 & 25 & 41 \\ 27 & 18 & 62 & 37 & 52 \\ 38 & 25 & 67 & 56 & 74 \\ -- & -- & -- & -- & 78\end{array}$

$\begin{array}{lllll}28 & 28 & 26 & 30 & 28\end{array}$

$\begin{array}{lllll}25 & 27 & 23 & 26 & 24\end{array}$

$\begin{array}{lllll}30 & 35 & 25 & 28 & 26\end{array}$

$\begin{array}{lllll}-- & -- & 27 & 33 & 30\end{array}$ 
Table 3: Cumulative percent ever starting a first union as a marriage, single-decrement life-table method with censoring at entry into cohabitation / Men

\begin{tabular}{|c|c|c|c|c|c|}
\hline age & $\begin{array}{c}\text { GDR } \\
(1984-89)\end{array}$ & $\begin{array}{l}\text { Hungary } \\
(1988-93)\end{array}$ & $\begin{array}{c}\text { Czech R } \\
\text { (NA) }\end{array}$ & $\begin{array}{c}\text { Slovenia } \\
(1989-95)\end{array}$ & $\begin{array}{c}\text { Latvia } \\
(1989-95\end{array}$ \\
\hline 16 & 0 & 0 & & 0 & 0 \\
\hline 18 & 0 & 0 & & 0 & 0 \\
\hline 20 & 2 & 3 & & 0 & 6 \\
\hline 22 & 11 & 12 & & 3 & 19 \\
\hline 24 & 24 & 29 & & 17 & 37 \\
\hline 25 & 34 & 37 & & 23 & 44 \\
\hline 26 & 41 & 44 & & 30 & 54 \\
\hline 28 & 48 & 55 & & 49 & 63 \\
\hline 30 & 53 & 61 & & 65 & 69 \\
\hline 35 & 59 & 67 & & 77 & -- \\
\hline 40 & -- & 68 & & -- & -- \\
\hline $\begin{array}{l}\text { mean age: } \\
\text { (at transition) }\end{array}$ & 25 & 25 & & 27 & 24 \\
\hline 1st decile at age: & 22 & 22 & & 23 & 21 \\
\hline 1st quartile at: & 25 & 24 & & 26 & 23 \\
\hline median at age: & 29 & 27 & & 29 & 26 \\
\hline 3rd quartile at: & -- & -- & & 33 & -- \\
\hline
\end{tabular}

$\begin{array}{cc}\text { Lithuania } & \text { Poland } \\ (1989-95) & \text { (NA) }\end{array}$

$16-0$

$18-1$

$20 \quad 8$

$22 \quad 25$

$24 \quad 50$

$25 \quad 59$

$26 \quad 70$

$28-80$

$30 \quad 86$

$35 \quad 93$

40 --

mean age: $\quad 24$

(at transition)

1st decile at age: $\quad 21$

1st quartile at: $\quad 22$

median at age: $\quad 24$

3rd quartile at: $\quad 27$ 
Table 3: Cumulative percent ever starting a first union as a marriage, single-decrement life-table method with censoring at entry into cohabitation / Women

\begin{tabular}{|c|c|c|c|c|c|}
\hline age & $\begin{array}{l}\text { Sweden } \\
(1978-93)\end{array}$ & $\begin{array}{c}\text { Norway } \\
(1974-89)\end{array}$ & $\begin{array}{l}\text { Finland } \\
(1979-89)\end{array}$ & $\begin{array}{c}\text { France } \\
(1988-94)\end{array}$ & $\begin{array}{c}\text { USA } \\
(1989-95)\end{array}$ \\
\hline 16 & 0 & 0 & 0 & 0 & 0 \\
\hline 18 & 0 & 2 & 0 & 1 & 3 \\
\hline 20 & 1 & 8 & 3 & 3 & 10 \\
\hline 22 & 2 & 19 & 8 & 5 & 21 \\
\hline 24 & 4 & 30 & 15 & 11 & 33 \\
\hline 25 & 4 & 36 & 19 & 17 & 38 \\
\hline 26 & 5 & 40 & 21 & 20 & 42 \\
\hline 28 & 6 & 48 & 26 & 25 & 51 \\
\hline 30 & 9 & 56 & 31 & 27 & 57 \\
\hline 35 & 12 & 65 & 39 & 31 & 66 \\
\hline 40 & 15 & 68 & 43 & 31 & 70 \\
\hline $\begin{array}{l}\text { mean age: } \\
\text { (at transition) }\end{array}$ & 29 & 25 & 27 & 26 & 25 \\
\hline 1st decile at age: & 31 & 21 & 23 & 24 & 20 \\
\hline 1st quartile at: & -- & 24 & 28 & 28 & 23 \\
\hline median at age: & -- & 29 & -- & -- & 28 \\
\hline 3rd quartile at: & -- & -- & -- & -- & -- \\
\hline ge & $\begin{array}{c}\text { Austria } \\
(1990-96)\end{array}$ & $\begin{array}{c}\text { West } \\
\text { Germany } \\
(1986-92)\end{array}$ & $\begin{array}{c}\text { Flanders } \\
(1985-92)\end{array}$ & $\begin{array}{c}\text { Italy } \\
(1990-95)\end{array}$ & $\begin{array}{c}\text { Spain } \\
(1989-95)\end{array}$ \\
\hline
\end{tabular}

$\begin{array}{lccccc}16 & 0 & 0 & 0 & 0 & 0 \\ 18 & 1 & 1 & 1 & 0 & 2 \\ 20 & 5 & 3 & 7 & 3 & 7 \\ 22 & 10 & 6 & 24 & 9 & 17 \\ 24 & 18 & 12 & 48 & 19 & 29 \\ 25 & 21 & 14 & 58 & 25 & 37 \\ 26 & 27 & 16 & 65 & 33 & 45 \\ 28 & 39 & 22 & 75 & 47 & 61 \\ 30 & 45 & 24 & 78 & 56 & 71 \\ 35 & 57 & 32 & 81 & 73 & 83 \\ 40 & 59 & -- & -- & 77 & 85 \\ & & & & & \\ \text { mean age: } & 27 & 27 & 24 & 27 & 26 \\ \text { (at transition) } & & & & & \\ & & & & & \\ \text { 1st decile at age: } & 22 & 24 & 21 & 23 & 21 \\ \text { 1st quartile at: } & 26 & 31 & 23 & 25 & 24 \\ \text { median at age: } & 32 & -- & 25 & 29 & 27 \\ \text { 3rd quartile at: } & -- & -- & 28 & 37 & 32\end{array}$


Table 3: Cumulative percent ever starting a first union as a marriage, single-decrement life-table method with censoring at entry into cohabitation / Women

\begin{tabular}{|c|c|c|c|c|c|}
\hline age & $\begin{array}{c}\text { GDR } \\
(1984-89)\end{array}$ & $\begin{array}{c}\text { Hungary } \\
(1988-93)\end{array}$ & $\begin{array}{c}\text { Czech R } \\
(1992-97)\end{array}$ & $\begin{array}{c}\text { Slovenia } \\
(1989-95)\end{array}$ & $\begin{array}{c}\text { Latvia } \\
(1989-95)\end{array}$ \\
\hline 16 & 0 & 0 & 0 & 0 & 0 \\
\hline 18 & 1 & 5 & 2 & 1 & 3 \\
\hline 20 & 12 & 24 & 17 & 8 & 22 \\
\hline 22 & 32 & 46 & 34 & 25 & 36 \\
\hline 24 & 49 & 65 & 49 & 42 & 45 \\
\hline 25 & 53 & 71 & 60 & 52 & 48 \\
\hline 26 & 56 & 75 & 65 & 59 & 55 \\
\hline 28 & 62 & 78 & 68 & 70 & 60 \\
\hline 30 & 65 & 79 & 74 & 73 & 66 \\
\hline 35 & 69 & 82 & -- & 82 & 71 \\
\hline 40 & -- & 84 & -- & -- & 73 \\
\hline $\begin{array}{l}\text { mean age: } \\
\text { (at transition) }\end{array}$ & 23 & 22 & 23 & 24 & 23 \\
\hline 1st decile at age: & 20 & 19 & 20 & 21 & 19 \\
\hline 1st quartile at: & 22 & 21 & 21 & 22 & 21 \\
\hline median at age: & 25 & 23 & 25 & 25 & 26 \\
\hline 3rd quartile at: & -- & 26 & 32 & 32 & -- \\
\hline age & $\begin{array}{l}\text { Lithuania } \\
(1989-95)\end{array}$ & $\begin{array}{l}\text { Poland } \\
(1986-91)\end{array}$ & & & \\
\hline 16 & 0 & 0 & & & \\
\hline 18 & 4 & 4 & & & \\
\hline 20 & 26 & 21 & & & \\
\hline 22 & 51 & 46 & & & \\
\hline 24 & 69 & 65 & & & \\
\hline 25 & 74 & 72 & & & \\
\hline 26 & 79 & 76 & & & \\
\hline 28 & 85 & 83 & & & \\
\hline 30 & 89 & 85 & & & \\
\hline 35 & 92 & 88 & & & \\
\hline 40 & 92 & 90 & & & \\
\hline $\begin{array}{l}\text { mean age: } \\
\text { (at transition) }\end{array}$ & 22 & 23 & & & \\
\hline 1st decile at age: & 19 & 19 & & & \\
\hline 1st quartile at: & 20 & 21 & & & \\
\hline median at age: & 22 & 23 & & & \\
\hline 3rd quartile at: & 26 & 26 & & & \\
\hline
\end{tabular}


Table 4: Cumulative percent ever starting a first union as a cohabitation,

competing-risks life-table method with direct marriage as a competing event / Men

$\begin{array}{cccccc}\text { age } & \text { Sweden } & \text { Norway } & \text { Finland } & \text { France } & \text { USA } \\ (1978-93) & (1974-89) & (1983-92) & (1988-94) & \text { (NA) }\end{array}$

16

18

20

22

24

25

26

28

30

35

40

mean age:

(at transition)

1st decile at age:

1st quartile at:

median at age:

3rd quartile at:

$\begin{array}{llll}0 & 0 & 0 & 0\end{array}$

$\begin{array}{llll}3 & 1 & 3 & 3\end{array}$

$\begin{array}{llll}16 & 8 & 9 & 10\end{array}$

$\begin{array}{llll}40 & 24 & 23 & 21\end{array}$

$\begin{array}{llll}59 & 42 & 43 & 38\end{array}$

$\begin{array}{llll}66 & 50 & 49 & 46\end{array}$

$\begin{array}{llll}72 & 55 & 56 & 53\end{array}$

$\begin{array}{llll}78 & 64 & 64 & 61\end{array}$

$\begin{array}{llll}83 & 66 & 70 & 65\end{array}$

$\begin{array}{llll}88 & 70 & 75 & 72\end{array}$

$\begin{array}{llll}89 & 71 & 77 & 74\end{array}$

$\begin{array}{llll}23 & 24 & 24 & 24\end{array}$

$\begin{array}{lccc}20 & 21 & 21 & 20 \\ 21 & 23 & 23 & 23 \\ 23 & 25 & 26 & 26 \\ 27 & -- & 34 & --\end{array}$

age

West

Austria Germany Flanders Italy Spain (1990-96) (1986-92) (1985-92) $\quad(1990-95) \quad(1989-95)$

16

18

20

22

24

25

26

28

30

35

40

$0 \quad 0$

20

124

$22 \quad 10$

$35 \quad 19$

$39 \quad 24$

$44 \quad 27$

$52 \quad 32$

$60 \quad 38$

$67 \quad 43$

0

$\begin{array}{lll}0 & 0 & 0\end{array}$

$\begin{array}{lll}1 & 0 & 0\end{array}$

$\begin{array}{lll}3 & 1 & 2\end{array}$

$\begin{array}{lll}7 & 1 & 4\end{array}$

15

20

22

28

32

35

9

12

15

mean age:

24

25

25

30

25

(at transition)

1st decile at age:

1st quartile at:

20

20

22

23

$34 \quad 26$

median at age:

28

3rd quartile at: 
Table 4: Cumulative percent ever starting a first union as a cohabitation, competing-risks life-table method with direct marriage as a competing event / Men

\begin{tabular}{|c|c|c|c|c|c|}
\hline age & $\begin{array}{c}\text { GDR } \\
(1984-89)\end{array}$ & $\begin{array}{l}\text { Hungary } \\
(1988-93)\end{array}$ & $\begin{array}{c}\text { Czech R } \\
\text { (NA) }\end{array}$ & $\begin{array}{c}\text { Slovenia } \\
(1989-95)\end{array}$ & $\begin{array}{c}\text { Latvia } \\
(1989-95\end{array}$ \\
\hline 16 & 0 & 0 & & 0 & 0 \\
\hline 18 & 1 & 1 & & 0 & 3 \\
\hline 20 & 7 & 4 & & 3 & 10 \\
\hline 22 & 16 & 11 & & 16 & 22 \\
\hline 24 & 30 & 17 & & 28 & 33 \\
\hline 25 & 37 & 21 & & 34 & 37 \\
\hline 26 & 42 & 24 & & 40 & 38 \\
\hline 28 & 48 & 27 & & 49 & 42 \\
\hline 30 & 52 & 28 & & 51 & 42 \\
\hline 35 & 55 & 31 & & 56 & -- \\
\hline 40 & -- & 33 & & -- & -- \\
\hline $\begin{array}{l}\text { mean age: } \\
\text { (at transition) }\end{array}$ & 24 & 25 & & 25 & 22 \\
\hline 1st decile at age: & 21 & 22 & & 21 & 20 \\
\hline 1st quartile at: & 24 & 27 & & 24 & 23 \\
\hline median at age: & 29 & -- & & 29 & -- \\
\hline 3rd quartile at: & -- & -- & & -- & -- \\
\hline
\end{tabular}

$\begin{array}{ccc}\text { Lithuania } & \text { Poland } \\ (1989-95) & \text { (NA) }\end{array}$

$16-0$

$18-1$

$20 \quad 3$

$22-11$

$24-16$

$25-17$

$26-18$

$28-20$

$30 \quad 21$

$35-22$

40 - --

mean age: $\quad 23$

(at transition)

1st decile at age: 22

1st quartile at: --

median at age: --

3rd quartile at: -- 
Table 4: Cumulative percent ever starting a first union as a cohabitation, competing-risks life-table method with direct marriage as a competing event / Women

\begin{tabular}{|c|c|c|c|c|c|}
\hline age & $\begin{array}{l}\text { Sweden } \\
(1978-93)\end{array}$ & $\begin{array}{c}\text { Norway } \\
(1974-89)\end{array}$ & $\begin{array}{l}\text { Finland } \\
(1979-89)\end{array}$ & $\begin{array}{c}\text { France } \\
(1988-94)\end{array}$ & $\begin{array}{c}\text { USA } \\
(1989-95)\end{array}$ \\
\hline 16 & 2 & 1 & 1 & 0 & 1 \\
\hline 18 & 12 & 8 & 7 & 4 & 8 \\
\hline 20 & 38 & 26 & 25 & 19 & 22 \\
\hline 22 & 62 & 45 & 48 & 38 & 36 \\
\hline 24 & 76 & 57 & 63 & 56 & 43 \\
\hline 25 & 80 & 61 & 69 & 62 & 46 \\
\hline 26 & 83 & 64 & 72 & 67 & 48 \\
\hline 28 & 87 & 67 & 76 & 71 & 52 \\
\hline 30 & 90 & 69 & 78 & 73 & 54 \\
\hline 35 & 92 & 70 & 80 & 77 & 56 \\
\hline 40 & 93 & 70 & 81 & 78 & 57 \\
\hline $\begin{array}{l}\text { mean age: } \\
\text { (at transition) }\end{array}$ & 21 & 22 & 22 & 23 & 22 \\
\hline 1st decile at age: & 18 & 19 & 19 & 19 & 19 \\
\hline 1st quartile at: & 20 & 20 & 20 & 21 & 21 \\
\hline median at age: & 21 & 23 & 23 & 24 & 27 \\
\hline 3rd quartile at: & 24 & -- & 28 & 32 & -- \\
\hline ye & $\begin{array}{c}\text { Austria } \\
(1990-96)\end{array}$ & $\begin{array}{c}\text { West } \\
\text { Germany } \\
(1986-92)\end{array}$ & $\begin{array}{c}\text { Flanders } \\
(1985-92)\end{array}$ & $\begin{array}{c}\text { Italy } \\
(1990-95)\end{array}$ & $\begin{array}{c}\text { Spain } \\
(1989-95)\end{array}$ \\
\hline
\end{tabular}

$\begin{array}{lccccc}16 & 1 & 0 & 0 & 0 & 0 \\ 18 & 7 & 2 & 1 & 1 & 2 \\ 20 & 22 & 11 & 6 & 2 & 5 \\ 22 & 40 & 20 & 13 & 4 & 6 \\ 24 & 53 & 32 & 19 & 5 & 9 \\ 25 & 57 & 37 & 22 & 6 & 12 \\ 26 & 61 & 40 & 25 & 6 & 14 \\ 28 & 67 & 48 & 28 & 8 & 17 \\ 30 & 71 & 53 & 29 & 10 & 18 \\ 35 & 74 & 57 & 31 & 13 & 20 \\ 40 & 74 & -- & -- & 14 & 20 \\ \text { mean age: } & & & & & \\ \text { (at transition) } & 22 & 24 & 23 & 26 & 24 \\ & & & & & \\ \text { 1st decile at age: } & & & & & \\ \text { 1st quartile at: } & 19 & 20 & 22 & 30 & 25 \\ \text { median at age: } & 21 & 23 & 26 & -- & -- \\ \text { 3rd quartile at: } & 24 & 29 & -- & -- & --\end{array}$


Table 4: Cumulative percent ever starting a first union as a cohabitation, competing-risks life-table method with direct marriage as a competing event / Women

$\begin{array}{lccccc}\text { age } & \begin{array}{c}\text { GDR } \\ (1984-89)\end{array} & \begin{array}{c}\text { Hungary } \\ (1988-93)\end{array} & \begin{array}{c}\text { Czech R } \\ (1992-97)\end{array} & \begin{array}{c}\text { Slovenia } \\ (1989-95)\end{array} & \begin{array}{c}\text { Latvia } \\ (1989-95)\end{array} \\ 16 & & & & & \\ 18 & 0 & 1 & 1 & 1 & 1 \\ 20 & 2 & 5 & 4 & 5 & 12 \\ 22 & 16 & 12 & 23 & 17 & 26 \\ 24 & 30 & 19 & 31 & 29 & 35 \\ 25 & 38 & 24 & 39 & 40 & 42 \\ 26 & 40 & 25 & 42 & 44 & 44 \\ 28 & 43 & 26 & 44 & 46 & 48 \\ 30 & 46 & 27 & 46 & 50 & 51 \\ 35 & 48 & 28 & 48 & 52 & 53 \\ 40 & 50 & 29 & -- & 53 & 54 \\ & -- & 30 & -- & -- & 54 \\ \text { mean age: } & & & & & \\ \text { (at transition) } & 22 & 22 & 21 & 22 & 21 \\ & & & & & \\ \text { 1st decile at age: } & 20 & 20 & 19 & 19 & 18 \\ \text { 1st quartile at: } & 22 & 25 & 21 & 21 & 20 \\ \text { median at age: } & 33 & -- & -- & 28 & 28 \\ \text { 3rd quartile at: } & -- & -- & -- & -- & --\end{array}$

$\begin{array}{ccc}\text { Lithuania } & \text { Poland } \\ & (1989-95) & (1986-91)\end{array}$

$\begin{array}{lcc}16 & 0 & 0 \\ 18 & 3 & 0 \\ 20 & 9 & 1 \\ 22 & 15 & 2 \\ 24 & 16 & 2 \\ 25 & 16 & 3 \\ 26 & 17 & 3 \\ 28 & 18 & 4 \\ 30 & 19 & 4 \\ 35 & 19 & 4 \\ 40 & 19 & 4 \\ & & \\ \text { mean age: } & 21 & 24 \\ \text { (at transition) } & & \\ & & \\ \text { 1st decile at age: } & 21 & -- \\ \text { 1st quartile at: } & -- & -- \\ \text { median at age: } & -- & -- \\ \text { 3rd quartile at: } & -- & --\end{array}$


Table 5: Cumulative percent ever starting a first union as a marriage, competing-risks life-table method with entry into cohabitation as a competing event / Men

age

16

18

20

22

24

25

26

28

30

35

40

mean age:

(at transition)

1st decile at age:

1st quartile at:

median at age:

3 rd quartile at:

$$
\begin{array}{ccccc}
\text { Sweden } & \text { Norway } & \text { Finland } & \text { France } & \text { USA } \\
(1978-93) & (1974-89) & (1983-92) & (1988-94) & \text { (NA) }
\end{array}
$$

age

16

18

20

22

24

25

26

28

30

35

40

mean age:

(at transition)

1st decile at age:

1st quartile at:

median at age:

3 rd quartile at:

\section{West}

Austria Germany Flanders Italy Spain (1990-96) (1986-92) (1985-92) (1990-95) (1989-95)

$\begin{array}{cccc}0 & 0 & 0 & 0 \\ 0 & 0 & 0 & 1 \\ 0 & 2 & 1 & 1 \\ 1 & 7 & 3 & 4 \\ 1 & 11 & 5 & 7 \\ 1 & 13 & 5 & 8 \\ 2 & 15 & 6 & 10 \\ 3 & 17 & 7 & 12 \\ 3 & 20 & 8 & 12 \\ 3 & 23 & 9 & 13 \\ 4 & 24 & 11 & 14\end{array}$

27

25

26

24

$\begin{array}{llll}-- & 24 & 36 & 26 \\ -- & -- & -- & -- \\ -- & -- & -- & -- \\ -- & -- & -- & --\end{array}$

0

0

0

$0 \quad 0$

0

0

$1 \quad 1$

$\begin{array}{lllll}25 & 27 & 23 & 26 & 24 \\ -- & -- & 25 & 29 & 27 \\ -- & -- & 32 & 34 & 31 \\ -- & -- & -- & -- & --\end{array}$


Table 5: Cumulative percent ever starting a first union as a marriage, competing-risks life-table method with entry into cohabitation as a competing event / Men

age

16

18

20

22

24

25

26

28

30

35

40

mean age:

(at transition)

1st decile at age:

1st quartile at:

median at age:

3rd quartile at:
GDR Hungary Czech R Slovenia Latvia

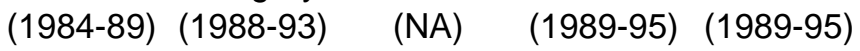

$0 \quad 0$

0

$0 \quad 0$

23

$9 \quad 11$

$19 \quad 25$

$26 \quad 32$

$29 \quad 37$

$32 \quad 44$

$34 \quad 48$

$35 \quad 51$

-- 52

24

25

26

23

$23 \quad 22$

$25 \quad 24$

24

27

21

$-$

32
0

6

17

28

32

38

42

$\begin{array}{ll}37 & 44 \\ 40 & --\end{array}$

$--\quad-$

age

16

18

20

22

24

25

26

28

30

35

40

mean age:

(at transition)

1st decile at age:

1st quartile at:

median at age:

3rd quartile at:
Lithuania Poland

(1989-95) (NA) 
Table 5: Cumulative percent ever starting a first union as a marriage, competing-risks life-table method with entry into cohabitation as a competing event / Women

\begin{tabular}{|c|c|c|c|c|c|}
\hline age & $\begin{array}{l}\text { Sweden } \\
(1978-93)\end{array}$ & $\begin{array}{c}\text { Norway } \\
(1974-89)\end{array}$ & $\begin{array}{l}\text { Finland } \\
(1979-89)\end{array}$ & $\begin{array}{c}\text { France } \\
(1988-94)\end{array}$ & $\begin{array}{c}\text { USA } \\
(1989-95)\end{array}$ \\
\hline 16 & 0 & 0 & 0 & 0 & 0 \\
\hline 18 & 0 & 1 & 0 & 1 & 3 \\
\hline 20 & 0 & 7 & 3 & 2 & 9 \\
\hline 22 & 1 & 13 & 6 & 4 & 16 \\
\hline 24 & 2 & 18 & 9 & 7 & 23 \\
\hline 25 & 2 & 20 & 10 & 9 & 25 \\
\hline 26 & 2 & 21 & 10 & 10 & 27 \\
\hline 28 & 2 & 23 & 11 & 12 & 31 \\
\hline 30 & 2 & 24 & 12 & 12 & 33 \\
\hline 35 & 3 & 26 & 13 & 13 & 35 \\
\hline 40 & 3 & 26 & 13 & 13 & 36 \\
\hline $\begin{array}{l}\text { mean age: } \\
\text { (at transition) }\end{array}$ & 25 & 23 & 24 & 23 & 23 \\
\hline 1st decile at age: & -- & 21 & 25 & 26 & 21 \\
\hline 1st quartile at: & -- & 31 & -- & -- & 25 \\
\hline median at age: & -- & -- & -- & -- & -- \\
\hline 3rd quartile at: & -- & -- & -- & -- & -- \\
\hline ge & $\begin{array}{c}\text { Austria } \\
(1990-96)\end{array}$ & $\begin{array}{c}\text { West } \\
\text { Germany } \\
(1986-92)\end{array}$ & $\begin{array}{c}\text { Flanders } \\
(1985-92)\end{array}$ & $\begin{array}{c}\text { Italy } \\
(1990-95)\end{array}$ & $\begin{array}{c}\text { Spain } \\
(1989-95)\end{array}$ \\
\hline
\end{tabular}

$\begin{array}{lccccc}16 & 0 & 0 & 0 & 0 & 0 \\ 18 & 1 & 1 & 1 & 0 & 2 \\ 20 & 4 & 3 & 7 & 3 & 7 \\ 22 & 8 & 6 & 22 & 8 & 16 \\ 24 & 11 & 9 & 41 & 18 & 27 \\ 25 & 13 & 11 & 49 & 24 & 34 \\ 26 & 15 & 12 & 53 & 32 & 41 \\ 28 & 18 & 15 & 59 & 44 & 54 \\ 30 & 20 & 16 & 60 & 52 & 61 \\ 35 & 21 & 19 & 62 & 66 & 69 \\ 40 & 22 & -- & -- & 69 & 70 \\ \text { mean age: } & & & & & \\ \text { (at transition) } & 24 & 25 & 23 & 27 & 25 \\ & & & & & \\ \text { 1st decile at age: } & & & & & \\ \text { 1st quartile at: } & 23 & 25 & 21 & 23 & 21 \\ \text { median at age: } & -- & -- & 23 & 26 & 24 \\ \text { 3rd quartile at: } & -- & -- & 26 & 29 & 28 \\ & -- & -- & -- & -- & --\end{array}$


Table 5: Cumulative percent ever starting a first union as a marriage, competing-risks life-table method with entry into cohabitation as a competing event / Women

GDR Hungary Czech R Slovenia Latvia $\begin{array}{lllll}(1984-89) & (1988-93) & (1992-97) & (1989-95) & (1989-95)\end{array}$

0

0
4

0

0

14

$11 \quad 22$

$26 \quad 40$

$35 \quad 53$

$38 \quad 57$

$39 \quad 59$

$41 \quad 61$

$42 \quad 62$

$43 \quad 63$

-- 64

22

22

$20 \quad 19$

$22 \quad 21$

$\begin{array}{ll}-- & 24\end{array}$

$--$

2

18

27

32

33

36

38

39

40

41

22

$\begin{array}{lll}22 & 23 & 22\end{array}$

age

16

18

20

22

24

25

26

28

30

35

40

mean age:

(at transition)

1st decile at age:

1st quartile at:

median at age:

3 rd quartile at:
Lithuania Poland

(1989-95) (1986-91)

16

18

$0 \quad 0$

34

$24 \quad 20$

$46 \quad 45$

$60 \quad 64$

$64 \quad 71$

$\begin{array}{ll}67 & 74\end{array}$

$72 \quad 80$

$74 \quad 83$

$76 \quad 86$

$76 \quad 87$

$22 \quad 23$

mean age:

1st decile at age:

1st quartile at:

median at age:

$19 \quad 19$

$21 \quad 21$

$23 \quad 23$

$31 \quad 27$

3rd quartile at: 
=> Table 6: Cumulative percent ever in a union / Men

age

16

18

20

22

24

25

26

28

30

35

40

mean age:

(at transition)

1st decile at age:

1st quartile at:

median at age:

3 rd quartile at:

age

16

18

20

22

24

25

26

28

30

35

40

mean age:

(at transition)

1st decile at age:

1st quartile at:

median at age:

3rd quartile at:
Sweden Norway Finland France USA $\begin{array}{lllll}(1978-93) & (1974-89) & (1983-92) & (1988-94) & \text { (NA) }\end{array}$

$\begin{array}{cccc}0 & 0 & 0 & 0 \\ 3 & 1 & 3 & 3 \\ 16 & 10 & 10 & 10 \\ 41 & 30 & 25 & 25 \\ 61 & 54 & 48 & 45 \\ 68 & 63 & 54 & 54 \\ 73 & 70 & 62 & 63 \\ 81 & 80 & 72 & 73 \\ 86 & 86 & 78 & 77 \\ 91 & 93 & 84 & 85 \\ 93 & 95 & 88 & 87\end{array}$

$\begin{array}{llll}23 & 24 & 25 & 24\end{array}$

$\begin{array}{llll}20 & 20 & 20 & 20 \\ 21 & 22 & 22 & 22 \\ 23 & 24 & 25 & 25 \\ 27 & 27 & 29 & 29\end{array}$

West

Austria Germany Flanders Italy Spain (1990-96) (1986-92) (1985-92) $\quad(1990-95) \quad(1989-95)$

$\begin{array}{ccccc}0 & 0 & 0 & 0 & 0 \\ 2 & 1 & 1 & 0 & 0 \\ 14 & 5 & 3 & 1 & 3 \\ 26 & 12 & 12 & 2 & 9 \\ 42 & 24 & 37 & 8 & 19 \\ 49 & 29 & 50 & 13 & 26 \\ 55 & 34 & 59 & 17 & 35 \\ 65 & 42 & 71 & 29 & 52 \\ 76 & 51 & 80 & 42 & 62 \\ 86 & 61 & 86 & 65 & 81 \\ -- & -- & -- & 78 & 84\end{array}$

$\begin{array}{lllll}25 & 26 & 25 & 30 & 27\end{array}$

$\begin{array}{lllll}20 & 22 & 22 & 25 & 23 \\ 22 & 25 & 24 & 28 & 25 \\ 26 & 30 & 25 & 31 & 28 \\ 30 & -- & 29 & 39 & 34\end{array}$


=> Table 6: Cumulative percent ever in a union / Men

age

16

18

20

22

24

25

26

28

30

35

40

mean age:

(at transition)

1st decile at age:

1st quartile at:

median at age:

3rd quartile at:

age

16

18

20

22

24

25

26

28

30

35

40

mean age:

(at transition)

1st decile at age:

1st quartile at:

median at age:

3rd quartile at:
GDR Hungary Czech R Slovenia Latvia $\begin{array}{llll}(1984-89) & (1988-93) \quad(N A) \quad(1989-95) & (1989-95)\end{array}$

$0 \quad 0$

$2 \quad 2$

98

$26 \quad 22$

$49 \quad 43$

$63 \quad 53$

$72 \quad 60$

$81 \quad 71$

$86 \quad 76$

$91 \quad 82$

$--\quad 85$

24

25

$\begin{array}{llll}21 & 21 & 21 & 20 \\ 22 & 23 & 23 & 21 \\ 25 & 25 & 25 & 23 \\ 27 & 30 & 28 & 26\end{array}$

Lithuania Poland

(1989-95) (NA)
25

23

38

$\begin{array}{ll}42 & 61 \\ 52 & 70\end{array}$

$62 \quad 76$

$80 \quad 84$

$88 \quad 87$

$96 \quad--$

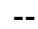

20

23

26
0

2

11

34

60

69

78

87

91

96

--

24

20

22

24

26 
=> Table 6: Cumulative percent ever in a union / Women

age

16

18

20

22

24

25

26

28

30

35

40

mean age:

(at transition)

1st decile at age:

1st quartile at:

median at age:

3rd quartile at:

age

16

18

20

22

24

25

26

28

30

35

40

mean age:

(at transition)

1st decile at age:

1st quartile at:

median at age:

3rd quartile at:
Sweden Norway Finland France USA $\begin{array}{lllll}(1978-93) & (1974-89) & (1979-89) & (1988-94) & (1989-95)\end{array}$

$\begin{array}{ccccc}2 & 1 & 1 & 0 & 2 \\ 12 & 10 & 7 & 5 & 10 \\ 39 & 32 & 27 & 22 & 31 \\ 63 & 59 & 54 & 42 & 52 \\ 77 & 75 & 72 & 63 & 66 \\ 81 & 81 & 78 & 72 & 72 \\ 85 & 85 & 82 & 77 & 75 \\ 89 & 90 & 87 & 83 & 83 \\ 92 & 93 & 90 & 85 & 86 \\ 94 & 96 & 94 & 90 & 91 \\ 96 & 96 & 95 & 91 & 93 \\ & & & & \\ 22 & 22 & 22 & 23 & 22 \\ & & & & \\ & & & & \\ 18 & 18 & 19 & 19 & 18 \\ 20 & 20 & 20 & 21 & 20 \\ 21 & 22 & 22 & 23 & 22 \\ 24 & 24 & 25 & 26 & 26\end{array}$

West

Austria Germany Flanders Italy Spain (1990-96) (1986-92) (1985-92) $\quad(1990-95) \quad(1989-95)$

$\begin{array}{ccccc}1 & 0 & 0 & 0 & 0 \\ 8 & 3 & 2 & 2 & 3 \\ 26 & 13 & 13 & 5 & 12 \\ 48 & 26 & 36 & 12 & 22 \\ 65 & 42 & 60 & 23 & 37 \\ 70 & 48 & 71 & 30 & 46 \\ 76 & 52 & 78 & 38 & 55 \\ 85 & 63 & 87 & 52 & 71 \\ 91 & 69 & 89 & 62 & 80 \\ 95 & 76 & 93 & 79 & 89 \\ 96 & -- & -- & 83 & 90\end{array}$

23

24

23

27

25

$\begin{array}{lllll}19 & 20 & 20 & 22 & 20 \\ 20 & 22 & 22 & 25 & 23 \\ 23 & 26 & 24 & 28 & 26 \\ 26 & 34 & 26 & 33 & 29\end{array}$


=> Table 6: Cumulative percent ever in a union / Women

age

16

18

20

22

24

25

26

28

30

35

40

mean age:

(at transition)

1st decile at age:

1st quartile at:

median at age:

3 rd quartile at:

age

16

18

20

22

24

25

26

28

30

35

40

mean age:

(at transition)

1st decile at age:

1st quartile at:

median at age:

3rd quartile at:
GDR Hungary Czech R Slovenia Latvia

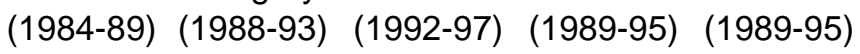

$\begin{array}{ccccc}0 & 1 & 1 & 1 & 1 \\ 3 & 10 & 6 & 6 & 14 \\ 27 & 34 & 37 & 24 & 43 \\ 55 & 59 & 57 & 49 & 62 \\ 74 & 77 & 74 & 70 & 74 \\ 78 & 82 & 82 & 79 & 78 \\ 82 & 85 & 86 & 84 & 85 \\ 87 & 88 & 90 & 92 & 89 \\ 90 & 90 & 93 & 94 & 92 \\ 93 & 92 & -- & 97 & 94 \\ -- & 94 & -- & -- & 95\end{array}$

$22 \quad 22$

21

22

21

$\begin{array}{lllll}19 & 18 & 19 & 19 & 18\end{array}$

$\begin{array}{lllll}20 & 20 & 20 & 21 & 19\end{array}$

$\begin{array}{lllll}22 & 22 & 22 & 23 & 21\end{array}$

$\begin{array}{lllll}25 & 24 & 25 & 25 & 25\end{array}$

Lithuania Poland

(1989-95) (1986-91)

$0 \quad 0$

$6 \quad 4$

$33 \quad 22$

$60 \quad 47$

$76 \quad 66$

$80 \quad 73$

$85 \quad 77$

$90 \quad 84$

$93 \quad 87$

$95 \quad 90$

$96 \quad 91$

$22 \quad 23$

$19 \quad 19$

$20 \quad 21$

$22 \quad 23$

$24 \quad 26$ 
Table 7: Cumulative percent ever married / Men

age

16

18

20

22

24

25

26

28

30

35

40

mean age:

(at transition)

1st decile at age:

1st quartile at:

median at age:

3rd quartile at:

age

16

18

20

22

24

25

26

28

30

35

40

mean age:

(at transition)

1st decile at age:

1st quartile at:

median at age:

3rd quartile at:
Sweden Norway Finland France USA $\begin{array}{lllll}(1978-93) & (1974-89) & (1983-92) & (1988-94) & \text { (NA) }\end{array}$

$\begin{array}{cccc}0 & 0 & 0 & 0 \\ 0 & 0 & 0 & 1 \\ 1 & 3 & 2 & 1 \\ 2 & 11 & 6 & 5 \\ 7 & 24 & 14 & 12 \\ 11 & 31 & 19 & 16 \\ 16 & 37 & 24 & 23 \\ 24 & 48 & 39 & 34 \\ 35 & 61 & 51 & 44 \\ 55 & 74 & 64 & 56 \\ 62 & 79 & 69 & 63\end{array}$

$\begin{array}{llll}29 & 27 & 28 & 28\end{array}$

$\begin{array}{cccc}25 & 22 & 23 & 24 \\ 29 & 25 & 27 & 27 \\ 33 & 29 & 30 & 33 \\ -- & 36 & -- & --\end{array}$

West

Austria Germany Flanders Italy Spain (1990-96) (1986-92) (1985-92) $\quad(1990-95) \quad(1989-95)$

$\begin{array}{ccccc}0 & 0 & 0 & 0 & 0 \\ 0 & 0 & 0 & 0 & 0 \\ 2 & 1 & 0 & 0 & 2 \\ 5 & 4 & 7 & 1 & 6 \\ 13 & 8 & 26 & 6 & 14 \\ 18 & 11 & 36 & 10 & 19 \\ 25 & 15 & 48 & 13 & 26 \\ 34 & 22 & 59 & 27 & 41 \\ 46 & 31 & 67 & 39 & 52 \\ 55 & 45 & 75 & 58 & 72 \\ 62 & 50 & -- & 72 & 78\end{array}$

$\begin{array}{lllll}28 & 29 & 26 & 30 & 28\end{array}$

$\begin{array}{lllll}23 & 25 & 23 & 25 & 23\end{array}$

$\begin{array}{lllll}26 & 29 & 24 & 28 & 26\end{array}$

$\begin{array}{lllll}32 & 39 & 27 & 33 & 30\end{array}$

$\begin{array}{lllll}32 & 39 & 27 & 33 & 30 \\ -- & -- & 34 & -- & 38\end{array}$


Table 7: Cumulative percent ever married / Men

age

16

18

20

22

24

25

26

28

30

35

40

mean age:

(at transition)

1st decile at age:

1st quartile at:

median at age:

3rd quartile at:

age

16

18

20

22

24

25

26

28

30

35

40

mean age:

(at transition)

1st decile at age:

1st quartile at:

median at age:

3rd quartile at:
GDR Hungary Czech R Slovenia Latvia $\begin{array}{llll}(1984-89) & (1988-93) \quad(N A) \quad(1989-95) & (1989-95)\end{array}$

$\begin{array}{ll}0 & 0\end{array}$

$0-0$

$3 \quad 4$

$14 \quad 15$

$32 \quad 35$

$44 \quad 42$

$52 \quad 51$

$63 \quad 62$

$70 \quad 69$

$78 \quad 76$

$\begin{array}{ll}78 & 77\end{array}$

25

25

$22 \quad 22$

22
23

$24 \quad 23$

$33 \quad 34$
$23 \quad 21$

$25 \quad 22$

$27 \quad 25$

$32 \quad 30$

26

48

56

63

70

76

79

$\begin{array}{ll}80 & 79 \\ 86 & --\end{array}$

$27 \quad 24$

21
25
30


Table 7: Cumulative percent ever married / Women

age

16

18

20

22

24

25

26

28

30

35

40

mean age:

(at transition)

1st decile at age:

1st quartile at:

median at age:

3 rd quartile at:

age

16

18

20

22

24

25

26

28

30

35

40

mean age:

(at transition)

1st decile at age:

1st quartile at:

median at age:

3rd quartile at:
Sweden Norway Finland France USA $\begin{array}{lllll}(1978-93) & (1974-89) & (1979-89) & (1988-94) & (1989-95)\end{array}$

$\begin{array}{ccccc}0 & 0 & 0 & 0 & 0 \\ 0 & 2 & 2 & 1 & 4 \\ 2 & 11 & 8 & 7 & 16 \\ 8 & 28 & 21 & 13 & 31 \\ 16 & 42 & 36 & 22 & 46 \\ 20 & 49 & 43 & 28 & 52 \\ 28 & 55 & 50 & 35 & 57 \\ 38 & 64 & 60 & 45 & 66 \\ 46 & 71 & 67 & 50 & 72 \\ 60 & 80 & 76 & 64 & 82 \\ 70 & 83 & 79 & 68 & 84\end{array}$

$\begin{array}{lllll}28 & 25 & 25 & 27 & 24\end{array}$

$\begin{array}{lllll}23 & 20 & 21 & 22 & 20 \\ 26 & 22 & 23 & 25 & 22 \\ 31 & 26 & 26 & 30 & 25 \\ -- & 32 & 34 & -- & 31\end{array}$

West

Austria Germany Flanders Italy Spain (1990-96) (1986-92) (1985-92) (1990-95) (1989-95)

$\begin{array}{ccccc}0 & 0 & 0 & 0 & 0 \\ 2 & 2 & 1 & 0 & 2 \\ 7 & 5 & 8 & 4 & 8 \\ 16 & 13 & 26 & 11 & 17 \\ 32 & 21 & 48 & 21 & 30 \\ 37 & 26 & 58 & 27 & 38 \\ 43 & 32 & 65 & 36 & 46 \\ 59 & 40 & 76 & 49 & 61 \\ 67 & 46 & 80 & 58 & 71 \\ 77 & 55 & 85 & 76 & 82 \\ 81 & 59 & -- & 81 & 85\end{array}$

$26 \quad 26$

$24 \quad 27$

26

$\begin{array}{lllll}21 & 22 & 21 & 22 & 21 \\ 23 & 25 & 22 & 25 & 24 \\ 27 & 32 & 25 & 29 & 27 \\ 33 & -- & 28 & 35 & 32\end{array}$


Table 7: Cumulative percent ever married / Women

age

16

18

20

22

24

25

26

28

30

35

40

mean age:

(at transition)

1st decile at age:

1st quartile at:

median at age:

3rd quartile at:

age

18

20

22

24

25

26

28

30

35

40

mean age:

(at transition)

1st decile at age:

1st quartile at:

median at age:

3rd quartile at:
GDR Hungary Czech R Slovenia Latvia $\begin{array}{lllll}(1984-89) & (1988-93) & (1992-97) & (1989-95) & (1989-95)\end{array}$

$\begin{array}{ccccc}0 & 0 & 0 & 0 & 0 \\ 1 & 5 & 3 & 1 & 5 \\ 15 & 26 & 22 & 11 & 29 \\ 38 & 48 & 44 & 31 & 49 \\ 58 & 67 & 62 & 50 & 61 \\ 63 & 74 & 71 & 58 & 65 \\ 67 & 78 & 76 & 64 & 70 \\ 74 & 82 & 82 & 74 & 75 \\ 77 & 84 & 86 & 79 & 79 \\ 81 & 88 & -- & 83 & 83 \\ -- & 89 & -- & 85 & 84\end{array}$

$23 \quad 22$

23

24

22

$\begin{array}{lllll}20 & 19 & 20 & 20 & 19\end{array}$

$21 \quad 20$

21

19

$24 \quad 23$

22

20

29

23

26

28
Lithuania Poland

(1989-95) (1986-91)

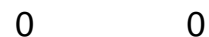

54

$29 \quad 21$

$55 \quad 46$

$72 \quad 65$

$77 \quad 72$

$81 \quad 76$

$87 \quad 83$

$91 \quad 85$

$93 \quad 89$

$94 \quad 90$

$22 \quad 23$

$19 \quad 19$

$20 \quad 21$

$22 \quad 23$

$25 \quad 26$ 
Table 8: Cumulative percent ever parent (including adoptions) / Men

\begin{tabular}{|c|c|c|c|c|c|}
\hline age & $\begin{array}{l}\text { Sweden } \\
(1978-93)\end{array}$ & $\begin{array}{c}\text { Norway } \\
(1974-89)\end{array}$ & $\begin{array}{l}\text { Finland } \\
(1983-92)\end{array}$ & $\begin{array}{c}\text { France } \\
(1988-94)\end{array}$ & $\begin{array}{l}\text { USA } \\
\text { (NA) }\end{array}$ \\
\hline 16 & 0 & 0 & 0 & 0 & \\
\hline 18 & 0 & 0 & 0 & 0 & \\
\hline 20 & 1 & 3 & 1 & 1 & \\
\hline 22 & 6 & 8 & 4 & 5 & \\
\hline 24 & 15 & 19 & 9 & 9 & \\
\hline 25 & 21 & 27 & 15 & 12 & \\
\hline 26 & 26 & 34 & 19 & 17 & \\
\hline 28 & 40 & 48 & 30 & 35 & \\
\hline 30 & 54 & 61 & 45 & 50 & \\
\hline 35 & 74 & 78 & 65 & 68 & \\
\hline 40 & 79 & 83 & 73 & 76 & \\
\hline $\begin{array}{l}\text { mean age: } \\
\text { (at transition) }\end{array}$ & 28 & 27 & 29 & 29 & \\
\hline 1st decile at age: & 23 & 23 & 25 & 25 & \\
\hline 1st quartile at: & 26 & 25 & 28 & 27 & \\
\hline median at age: & 30 & 29 & 31 & 30 & \\
\hline 3rd quartile at: & 36 & 34 & -- & 38 & \\
\hline age & $\begin{array}{c}\text { Austria } \\
(1990-96)\end{array}$ & $\begin{array}{c}\text { West } \\
\text { Germany } \\
(1986-92)\end{array}$ & $\begin{array}{l}\text { Flanders } \\
\text { (1985-92) }\end{array}$ & $\begin{array}{c}\text { Italy } \\
(1990-95)\end{array}$ & $\begin{array}{c}\text { Spain } \\
(1989-95)\end{array}$ \\
\hline
\end{tabular}

$\begin{array}{lccccc}16 & 0 & 0 & 0 & 0 & 0 \\ 18 & 0 & 0 & 0 & 0 & 1 \\ 20 & 1 & 1 & 1 & 0 & 2 \\ 22 & 6 & 3 & 3 & 0 & 4 \\ 24 & 11 & 6 & 9 & 3 & 9 \\ 25 & 16 & 7 & 14 & 4 & 12 \\ 26 & 22 & 10 & 21 & 8 & 16 \\ 28 & 33 & 16 & 40 & 15 & 26 \\ 30 & 45 & 26 & 55 & 26 & 41 \\ 35 & 61 & 42 & 73 & 51 & 66 \\ 40 & 72 & 47 & 78 & 62 & 74 \\ & & & & & \\ \text { mean age: } & 29 & 29 & 28 & 31 & 29 \\ \text { (at transition) } & & & & & \\ & & & & & \\ \text { 1st decile at age: } & 24 & 26 & 25 & 27 & 25 \\ \text { 1st quartile at: } & 27 & 30 & 27 & 30 & 28 \\ \text { median at age: } & 32 & -- & 30 & 35 & 32 \\ \text { 3rd quartile at: } & -- & -- & 38 & -- & --\end{array}$


Table 8: Cumulative percent ever parent (including adoptions) / Men

\begin{tabular}{|c|c|c|c|c|c|}
\hline age & $\begin{array}{c}\text { GDR } \\
(1984-89)\end{array}$ & $\begin{array}{c}\text { Hungary } \\
(1988-93)\end{array}$ & $\begin{array}{c}\text { Czech R } \\
\text { (NA) }\end{array}$ & $\begin{array}{l}\text { Slovenia } \\
(1989-95)\end{array}$ & $\begin{array}{c}\text { Latvia } \\
(1989-95)\end{array}$ \\
\hline 16 & 0 & 0 & & 0 & 0 \\
\hline 18 & 2 & 0 & & 0 & 0 \\
\hline 20 & 4 & 4 & & 1 & 6 \\
\hline 22 & 16 & 9 & & 5 & 17 \\
\hline 24 & 32 & 25 & & 26 & 39 \\
\hline 25 & 41 & 34 & & 37 & 50 \\
\hline 26 & 50 & 41 & & 47 & 57 \\
\hline 28 & 64 & 55 & & 63 & 70 \\
\hline 30 & 72 & 63 & & 77 & 77 \\
\hline 35 & 83 & 75 & & 89 & 80 \\
\hline 40 & -- & 79 & & 93 & 82 \\
\hline $\begin{array}{l}\text { mean age: } \\
\text { (at transition) }\end{array}$ & 26 & 26 & & 27 & 25 \\
\hline 1st decile at age: & 22 & 23 & & 23 & 21 \\
\hline 1st quartile at: & 24 & 24 & & 24 & 23 \\
\hline median at age: & 26 & 28 & & 27 & 25 \\
\hline 3rd quartile at: & 31 & 35 & & 30 & 30 \\
\hline age & $\begin{array}{l}\text { Lithuania } \\
(1989-95)\end{array}$ & $\begin{array}{l}\text { Poland } \\
\text { (NA) }\end{array}$ & & & \\
\hline 16 & 0 & & & & \\
\hline 18 & 0 & & & & \\
\hline 20 & 5 & & & & \\
\hline 22 & 15 & & & & \\
\hline 24 & 38 & & & & \\
\hline 25 & 46 & & & & \\
\hline 26 & 55 & & & & \\
\hline 28 & 69 & & & & \\
\hline 30 & 79 & & & & \\
\hline 35 & 86 & & & & \\
\hline 40 & 88 & & & & \\
\hline $\begin{array}{l}\text { mean age: } \\
\text { (at transition) }\end{array}$ & 25 & & & & \\
\hline 1st decile at age: & 22 & & & & \\
\hline 1st quartile at: & 24 & & & & \\
\hline median at age: & 26 & & & & \\
\hline 3rd quartile at: & 29 & & & & \\
\hline
\end{tabular}


Table 8: Cumulative percent ever parent (including adoptions) / Women

\begin{tabular}{|c|c|c|c|c|c|}
\hline age & $\begin{array}{l}\text { Sweden } \\
(1978-93)\end{array}$ & $\begin{array}{c}\text { Norway } \\
(1974-89)\end{array}$ & $\begin{array}{l}\text { Finland } \\
(1979-89)\end{array}$ & $\begin{array}{c}\text { France } \\
(1988-94)\end{array}$ & $\begin{array}{c}\text { USA } \\
(1989-95)\end{array}$ \\
\hline 16 & 0 & 0 & 0 & 0 & 2 \\
\hline 18 & 1 & 3 & 3 & 2 & 8 \\
\hline 20 & 6 & 12 & 8 & 6 & 21 \\
\hline 22 & 15 & 27 & 18 & 13 & 33 \\
\hline 24 & 29 & 40 & 29 & 26 & 43 \\
\hline 25 & 36 & 47 & 36 & 33 & 47 \\
\hline 26 & 43 & 53 & 42 & 40 & 51 \\
\hline 28 & 57 & 66 & 57 & 53 & 59 \\
\hline 30 & 68 & 75 & 67 & 64 & 67 \\
\hline 35 & 83 & 85 & 78 & 79 & 78 \\
\hline 40 & 87 & 88 & 82 & 83 & 81 \\
\hline $\begin{array}{l}\text { mean age: } \\
\text { (at transition) }\end{array}$ & 26 & 25 & 26 & 27 & 24 \\
\hline 1st decile at age: & 21 & 20 & 21 & 22 & 19 \\
\hline 1st quartile at: & 24 & 22 & 24 & 24 & 21 \\
\hline median at age: & 27 & 26 & 27 & 28 & 26 \\
\hline 3rd quartile at: & 32 & 30 & 33 & 34 & 34 \\
\hline age & $\begin{array}{c}\text { Austria } \\
(1990-96)\end{array}$ & $\begin{array}{c}\text { West } \\
\text { Germany } \\
(1986-92)\end{array}$ & $\begin{array}{l}\text { Flanders } \\
\text { (1985-92) }\end{array}$ & $\begin{array}{c}\text { Italy } \\
(1990-95)\end{array}$ & $\begin{array}{c}\text { Spain } \\
(1989-95)\end{array}$ \\
\hline
\end{tabular}

$\begin{array}{lccccc}16 & 0 & 0 & 0 & 1 & 0 \\ 18 & 3 & 0 & 0 & 3 & 2 \\ 20 & 12 & 3 & 2 & 4 & 7 \\ 22 & 24 & 9 & 7 & 8 & 14 \\ 24 & 40 & 17 & 19 & 14 & 22 \\ 25 & 47 & 21 & 28 & 19 & 27 \\ 26 & 56 & 28 & 37 & 25 & 32 \\ 28 & 71 & 39 & 56 & 37 & 46 \\ 30 & 79 & 49 & 69 & 51 & 61 \\ 35 & 88 & 62 & 79 & 74 & 81 \\ 40 & 91 & 64 & 80 & 79 & 86 \\ & & & & & \\ \text { mean age: } & 25 & 27 & 26 & 28 & 27 \\ \text { (at transition) } & & & & & \\ \text { 1st decile at age: } & 20 & 23 & 23 & 23 & 21 \\ \text { 1st quartile at: } & 23 & 26 & 25 & 26 & 25 \\ \text { median at age: } & 26 & 31 & 28 & 30 & 29 \\ \text { 3rd quartile at: } & 29 & -- & 33 & 37 & 33\end{array}$


Table 8: Cumulative percent ever parent (including adoptions) / Women

\begin{tabular}{|c|c|c|c|c|c|}
\hline age & $\begin{array}{c}\text { GDR } \\
(1984-89)\end{array}$ & $\begin{array}{c}\text { Hungary } \\
(1988-93)\end{array}$ & $\begin{array}{c}\text { Czech R } \\
(1992-97)\end{array}$ & $\begin{array}{c}\text { Slovenia } \\
(1989-95)\end{array}$ & $\begin{array}{c}\text { Latvia } \\
(1989-95)\end{array}$ \\
\hline 16 & 0 & 0 & 0 & 0 & 0 \\
\hline 18 & 3 & 3 & 3 & 2 & 5 \\
\hline 20 & 15 & 17 & 19 & 12 & 22 \\
\hline 22 & 42 & 38 & 38 & 33 & 45 \\
\hline 24 & 63 & 57 & 53 & 57 & 60 \\
\hline 25 & 70 & 64 & 62 & 65 & 66 \\
\hline 26 & 77 & 71 & 69 & 74 & 72 \\
\hline 28 & 83 & 81 & 80 & 84 & 81 \\
\hline 30 & 87 & 84 & 84 & 89 & 85 \\
\hline 35 & 91 & 91 & 89 & 94 & 91 \\
\hline 40 & -- & 93 & -- & 96 & 92 \\
\hline $\begin{array}{l}\text { mean age: } \\
\text { (at transition) }\end{array}$ & 23 & 24 & 23 & 24 & 23 \\
\hline 1st decile at age: & 20 & 20 & 20 & 20 & 19 \\
\hline 1st quartile at: & 21 & 21 & 21 & 22 & 21 \\
\hline median at age: & 23 & 24 & 24 & 24 & 23 \\
\hline 3rd quartile at: & 26 & 27 & 27 & 27 & 27 \\
\hline age & $\begin{array}{l}\text { Lithuania } \\
(1989-95)\end{array}$ & $\begin{array}{l}\text { Poland } \\
(1986-91)\end{array}$ & & & \\
\hline 16 & 0 & 0 & & & \\
\hline 18 & 2 & 2 & & & \\
\hline 20 & 14 & 14 & & & \\
\hline 22 & 41 & 37 & & & \\
\hline 24 & 63 & 58 & & & \\
\hline 25 & 71 & 65 & & & \\
\hline 26 & 77 & 72 & & & \\
\hline 28 & 83 & 82 & & & \\
\hline 30 & 87 & 86 & & & \\
\hline 35 & 91 & 90 & & & \\
\hline 40 & 92 & 92 & & & \\
\hline $\begin{array}{l}\text { mean age: } \\
\text { (at transition) }\end{array}$ & 23 & 24 & & & \\
\hline 1st decile at age: & 20 & 20 & & & \\
\hline 1st quartile at: & 21 & 22 & & & \\
\hline median at age: & 23 & 24 & & & \\
\hline 3rd quartile at: & 26 & 27 & & & \\
\hline
\end{tabular}


Table 9: Cumulative percent ever "parent and in a union" / Men

age

16

18

20

22

24

25

26

28

30

35

40

mean age:

(at transition)

1st decile at age:

1st quartile at:

median at age:

3 rd quartile at:

age

16

18

20

22

24

25

26

28

30

35

40

mean age:

(at transition)

1st decile at age:

1st quartile at:

median at age:

3rd quartile at:
Sweden Norway Finland France USA

$\begin{array}{lllll}(1978-93) & (1974-89) & (1983-92) & (1988-94) & \text { (NA) }\end{array}$

$\begin{array}{cccc}0 & 0 & 0 & 0 \\ 0 & 0 & 0 & 0 \\ 1 & 2 & 1 & 1 \\ 6 & 8 & 4 & 5 \\ 15 & 18 & 9 & 9 \\ 20 & 27 & 15 & 12 \\ 26 & 34 & 19 & 18 \\ 39 & 48 & 30 & 35 \\ 53 & 61 & 45 & 50 \\ 72 & 77 & 65 & 68 \\ 77 & 82 & 72 & 76\end{array}$

$\begin{array}{llll}28 & 27 & 29 & 29\end{array}$

$\begin{array}{llll}23 & 23 & 25 & 25 \\ 26 & 25 & 28 & 27 \\ 30 & 29 & 31 & 30 \\ 37 & 34 & -- & 38\end{array}$

West

Austria Germany Flanders Italy Spain (1990-96) (1986-92) (1985-92) $\quad(1990-95) \quad(1989-95)$

$\begin{array}{ccccc}0 & 0 & 0 & 0 & 0 \\ 0 & 0 & 0 & 0 & 1 \\ 1 & 1 & 0 & 0 & 2 \\ 4 & 2 & 3 & 0 & 3 \\ 9 & 5 & 9 & 3 & 8 \\ 15 & 7 & 14 & 4 & 11 \\ 21 & 9 & 21 & 8 & 16 \\ 31 & 16 & 40 & 15 & 26 \\ 43 & 26 & 55 & 26 & 40 \\ 60 & 41 & 73 & 51 & 66 \\ 70 & 46 & 78 & 61 & 73\end{array}$

29

29

28

31

30

$\begin{array}{lllll}25 & 27 & 25 & 27 & 25 \\ 27 & 30 & 27 & 30 & 28 \\ 32 & -- & 30 & 35 & 32 \\ -- & -- & 38 & -- & --\end{array}$


Table 9: Cumulative percent ever "parent and in a union" / Men

age

16

18

20

22

24

25

26

28

30

35

40

mean age:

(at transition)

1st decile at age:

1st quartile at:

median at age:

3rd quartile at:

age

16

18

20

22

24

25

26

28

30

35

40

mean age:

(at transition)

1st decile at age:

1st quartile at:

median at age:

3rd quartile at:
GDR Hungary Czech R Slovenia Latvia $\begin{array}{llll}(1984-89) & (1988-93) \quad(N A) \quad(1989-95) & (1989-95)\end{array}$

0

$3-3$

$13 \quad 9$

$30 \quad 25$

$39 \quad 34$

$48 \quad 41$

$64 \quad 55$

$71 \quad 63$

$82 \quad 75$

$--\quad 79$

$26 \quad 26$

$22 \quad 23$

23

$24-24$

$27 \quad 28$

$32 \quad 35$

$\begin{array}{lll}0 & 0\end{array}$

$\begin{array}{ll}0 & 0 \\ 0 & 0\end{array}$

$\begin{array}{lll}31 & 47 & 57\end{array}$

$\begin{array}{lll}64 & 70\end{array}$

$\begin{array}{lll}77 & 76\end{array}$

$89-80$

$\begin{array}{lll}79 & 93 & 81\end{array}$

$\begin{array}{lll}26 & 27 & 25\end{array}$

$\begin{array}{ll}27 & 25 \\ 30 & 30\end{array}$

Lithuania Poland

(1989-95) (NA)

0
0
6
16
38
50
57
70
76
80
81

25


21
23
25
30

16


Table 9: Cumulative percent ever "parent and in a union" / Women

age

16

18

20

22

24

25

26

28

30

35

40

mean age:

(at transition)

1st decile at age:

1st quartile at:

median at age:

3 rd quartile at:

age

16

18

20

22

24

25

26

28

30

35

40

mean age:

(at transition)

1st decile at age:

1st quartile at:

median at age:

3rd quartile at:
Sweden Norway Finland France USA $\begin{array}{lllll}(1978-93) & (1974-89) & (1979-89) & (1988-94) & (1989-95)\end{array}$

$\begin{array}{ccccc}0 & 0 & 0 & 0 & 0 \\ 1 & 2 & 2 & 1 & 4 \\ 5 & 10 & 8 & 5 & 14 \\ 14 & 25 & 18 & 10 & 26 \\ 28 & 39 & 28 & 23 & 36 \\ 35 & 45 & 35 & 31 & 41 \\ 41 & 52 & 42 & 38 & 45 \\ 55 & 66 & 55 & 51 & 54 \\ 66 & 74 & 66 & 61 & 62 \\ 81 & 84 & 77 & 76 & 74 \\ 85 & 88 & 81 & 80 & 78\end{array}$

$\begin{array}{lllll}26 & 25 & 26 & 27 & 25\end{array}$

$\begin{array}{lllll}22 & 20 & 21 & 22 & 20 \\ 24 & 22 & 24 & 25 & 22 \\ 28 & 26 & 28 & 28 & 27 \\ 33 & 31 & 34 & 35 & 36\end{array}$

West

Austria Germany Flanders Italy Spain (1990-96) (1986-92) (1985-92) $\quad(1990-95) \quad(1989-95)$

$\begin{array}{ccccc}0 & 0 & 0 & 1 & 0 \\ 1 & 0 & 0 & 2 & 2 \\ 9 & 2 & 2 & 4 & 6 \\ 21 & 8 & 7 & 7 & 14 \\ 37 & 16 & 19 & 14 & 22 \\ 43 & 19 & 27 & 19 & 26 \\ 52 & 26 & 37 & 25 & 32 \\ 68 & 37 & 56 & 37 & 45 \\ 76 & 47 & 68 & 50 & 61 \\ 85 & 59 & 79 & 73 & 81 \\ 89 & 62 & 80 & 79 & 85\end{array}$

$\begin{array}{lllll}25 & 27 & 27 & 28 & 27\end{array}$

$\begin{array}{lllll}21 & 23 & 23 & 23 & 21 \\ 23 & 26 & 25 & 26 & 25 \\ 26 & 31 & 28 & 30 & 29 \\ 30 & -- & 33 & 37 & 33\end{array}$


Table 9: Cumulative percent ever "parent and in a union" / Women

age

16

18

20

22

24

25

26

28

30

35

40

mean age:

(at transition)

1st decile at age:

1st quartile at:

median at age:

3 rd quartile at:

age

16

18

20

22

24

25

26

28

30

35

40

mean age:

(at transition)

1st decile at age:

1st quartile at:

median at age:

3rd quartile at:
GDR Hungary Czech R Slovenia Latvia $\begin{array}{lllll}(1984-89) & (1988-93) & (1992-97) & (1989-95) & (1989-95)\end{array}$

$\begin{array}{ccccc}0 & 0 & 0 & 0 & 0 \\ 1 & 3 & 2 & 2 & 4 \\ 12 & 16 & 18 & 11 & 19 \\ 35 & 36 & 36 & 32 & 42 \\ 57 & 54 & 51 & 54 & 58 \\ 65 & 62 & 59 & 64 & 63 \\ 72 & 70 & 66 & 73 & 69 \\ 79 & 80 & 76 & 83 & 78 \\ 83 & 83 & 81 & 88 & 82 \\ 88 & 90 & 86 & 92 & 88 \\ -- & 92 & -- & 94 & 89\end{array}$

$23 \quad 24$

24

24

23

$\begin{array}{lllll}20 & 20 & 20 & 20 & 19 \\ 22 & 21 & 21 & 22 & 21 \\ 24 & 24 & 24 & 24 & 23 \\ 27 & 27 & 28 & 27 & 28\end{array}$

Lithuania Poland

(1989-95) (1986-91)

$0 \quad 0$

$2 \quad 2$

$14 \quad 12$

$39 \quad 32$

$62 \quad 53$

$68 \quad 60$

$75 \quad 67$

$81 \quad 76$

$85 \quad 80$

$90 \quad 84$

$90 \quad 87$

$23 \quad 24$

$20 \quad 20$

$21 \quad 22$

$23 \quad 24$

$26 \quad 28$ 
Table 10: Cumulative percent ever "parent and married" / Men

$\begin{array}{lcccc}\text { age } & \begin{array}{c}\text { Sweden } \\ (1978-93)\end{array} & \begin{array}{c}\text { Norway } \\ (1974-89)\end{array} & \begin{array}{c}\text { Finland } \\ (1983-92)\end{array} & \begin{array}{c}\text { France } \\ (1988-94)\end{array} \\ 16 & 0 & 0 & 0 & 0 \\ 18 & 0 & 0 & 0 & 0 \\ 20 & 0 & 2 & 0 & 0 \\ 22 & 1 & 6 & 2 & 3 \\ 24 & 5 & 14 & 6 & 5 \\ 25 & 7 & 21 & 10 & 8 \\ 26 & 11 & 27 & 13 & 12 \\ 28 & 17 & 39 & 22 & 25 \\ 30 & 29 & 53 & 37 & 38 \\ 35 & 51 & 70 & 58 & 54 \\ 40 & 60 & 76 & 65 & 63 \\ & & & & \\ \text { mean age: } & 30 & 28 & 29 & 29 \\ \text { (at transition) } & & & & \\ & & & & \\ \text { 1st decile at age: } & 26 & 24 & 25 & 26 \\ \text { 1st quartile at: } & 30 & 26 & 29 & 28 \\ \text { median at age: } & 35 & 30 & 33 & 34 \\ \text { 3rd quartile at: } & -- & 38 & -- & --\end{array}$

$\begin{array}{ccccc}\text { Austria } & \text { Germany } & \text { Flanders } & \text { Italy } & \text { Spain } \\ (1990-96) & (1986-92) & (1985-92) & (1990-95) & (1989-95)\end{array}$

$\begin{array}{lccccc}16 & 0 & 0 & 0 & 0 & 0 \\ 18 & 0 & 0 & 0 & 0 & 0 \\ 20 & 1 & 0 & 0 & 0 & 2 \\ 22 & 2 & 1 & 2 & 0 & 3 \\ 24 & 6 & 4 & 7 & 3 & 8 \\ 25 & 11 & 6 & 12 & 4 & 10 \\ 26 & 15 & 7 & 20 & 8 & 15 \\ 28 & 25 & 14 & 38 & 15 & 24 \\ 30 & 38 & 23 & 53 & 27 & 38 \\ 35 & 52 & 38 & 70 & 51 & 64 \\ 40 & 63 & 43 & 75 & 61 & 71 \\ & & & & & \\ \text { mean age: } & 29 & 30 & 28 & 31 & 30 \\ \text { (at transition) } & & & & & \\ & & & & & \\ \text { 1st decile at age: } & 25 & 27 & 25 & 27 & 25 \\ \text { 1st quartile at: } & 28 & 31 & 27 & 30 & 29 \\ \text { median at age: } & 34 & -- & 30 & 35 & 32 \\ \text { 3rd quartile at: } & -- & -- & 40 & -- & --\end{array}$


Table 10: Cumulative percent ever "parent and married" / Men

age

16

18

20

22

24

25

26

28

30

35

40

mean age:

(at transition)

1st decile at age:

1st quartile at:

median at age:

3 rd quartile at:

age

16

18

20

22

24

25

26

28

30

35

40

mean age:

(at transition)

1st decile at age:

1st quartile at:

median at age:

3rd quartile at:
GDR Hungary Czech R Slovenia Latvia $\begin{array}{llll}(1984-89) & (1988-93) \quad(N A) \quad(1989-95) & (1989-95)\end{array}$

0

$1-3$

$9 \quad 7$

$24 \quad 23$

$32 \quad 32$

$41 \quad 38$

$55 \quad 51$

$64 \quad 61$

$75 \quad 72$

$--\quad 76$

$26 \quad 27$

$23 \quad 23$

23

$25 \quad 25$

$28 \quad 28$

$35 \quad 39$

$\begin{array}{ll}29 & 26 \\ 34 & 33\end{array}$

Lithuania Poland

(1989-95) (NA)

15

36

47

54

65

72

75

76

$28 \quad 25$

$\begin{array}{ll}24 & 21 \\ 25 & 23 \\ 29 & 26 \\ 34 & 33\end{array}$

0

0

4

14

37

46

54

68

79

86

88

25

22

24

26

29 
Table 10: Cumulative percent ever "parent and married" / Women

\begin{tabular}{|c|c|c|c|c|c|}
\hline age & $\begin{array}{l}\text { Sweden } \\
(1978-93)\end{array}$ & $\begin{array}{l}\text { Norway } \\
(1974-89)\end{array}$ & $\begin{array}{l}\text { Finland } \\
(1979-89)\end{array}$ & $\begin{array}{c}\text { France } \\
(1988-94)\end{array}$ & $\begin{array}{c}\text { USA } \\
(1989-95)\end{array}$ \\
\hline 16 & 0 & 0 & 0 & 0 & 0 \\
\hline 18 & 0 & 1 & 1 & 0 & 2 \\
\hline 20 & 1 & 7 & 6 & 4 & 9 \\
\hline 22 & 5 & 18 & 14 & 6 & 18 \\
\hline 24 & 11 & 30 & 23 & 16 & 27 \\
\hline 25 & 15 & 37 & 29 & 21 & 32 \\
\hline 26 & 21 & 43 & 35 & 26 & 37 \\
\hline 28 & 31 & 56 & 49 & 37 & 46 \\
\hline 30 & 40 & 64 & 58 & 45 & 54 \\
\hline 35 & 56 & 76 & 70 & 60 & 68 \\
\hline 40 & 67 & 80 & 74 & 64 & 73 \\
\hline $\begin{array}{l}\text { mean age: } \\
\text { (at transition) }\end{array}$ & 29 & 26 & 26 & 28 & 26 \\
\hline 1st decile at age: & 24 & 21 & 22 & 23 & 21 \\
\hline 1st quartile at: & 27 & 24 & 25 & 26 & 24 \\
\hline median at age: & 32 & 27 & 29 & 32 & 29 \\
\hline 3rd quartile at: & -- & 35 & -- & -- & -- \\
\hline age & $\begin{array}{c}\text { Austria } \\
(1990-96)\end{array}$ & $\begin{array}{c}\text { West } \\
\text { Germany } \\
(1986-92)\end{array}$ & $\begin{array}{c}\text { Flanders } \\
(1985-92)\end{array}$ & $\begin{array}{c}\text { Italy } \\
(1990-95)\end{array}$ & $\begin{array}{c}\text { Spain } \\
(1989-95)\end{array}$ \\
\hline
\end{tabular}

$\begin{array}{lccccc}16 & 0 & 0 & 0 & 1 & 0 \\ 18 & 1 & 1 & 0 & 1 & 1 \\ 20 & 4 & 2 & 1 & 4 & 5 \\ 22 & 11 & 7 & 6 & 7 & 12 \\ 24 & 24 & 14 & 17 & 13 & 21 \\ 25 & 31 & 18 & 26 & 18 & 24 \\ 26 & 38 & 24 & 35 & 24 & 30 \\ 28 & 55 & 33 & 54 & 36 & 43 \\ 30 & 65 & 42 & 66 & 49 & 58 \\ 35 & 76 & 53 & 76 & 70 & 77 \\ 40 & 81 & 57 & 77 & 77 & 82 \\ & & & & & \\ \text { mean age: } & 27 & 27 & 27 & 28 & 27 \\ \text { (at transition) } & & & & & \\ \text { 1st decile at age: } & 22 & 23 & 23 & 23 & 22 \\ \text { 1st quartile at: } & 25 & 27 & 25 & 27 & 26 \\ \text { median at age: } & 28 & 33 & 28 & 31 & 30 \\ \text { 3rd quartile at: } & 35 & -- & 35 & 38 & 34\end{array}$


Table 10: Cumulative percent ever "parent and married" / Women

age

16

18

20

22

24

25

26

28

30

35

40

mean age:

(at transition)

1st decile at age:

1st quartile at:

median at age:

3 rd quartile at:

age

18

20

22

24

25

26

28

30

35

40

mean age:

(at transition)

1st decile at age:

1st quartile at:

median at age:

3rd quartile at:
GDR Hungary Czech $\mathrm{R}$ Slovenia Latvia $\begin{array}{lllll}(1984-89) & (1988-93) & (1992-97) & (1989-95) & (1989-95)\end{array}$

$\begin{array}{ccccc}0 & 0 & 0 & 0 & 0 \\ 1 & 2 & 1 & 1 & 3 \\ 8 & 14 & 15 & 8 & 17 \\ 28 & 34 & 34 & 24 & 38 \\ 49 & 52 & 50 & 44 & 53 \\ 56 & 60 & 57 & 52 & 58 \\ 62 & 68 & 64 & 59 & 63 \\ 70 & 77 & 74 & 70 & 70 \\ 74 & 81 & 79 & 76 & 74 \\ 79 & 87 & 84 & 81 & 80 \\ -- & 90 & 86 & 83 & 81\end{array}$

$24 \quad 24$

24

24

23

$\begin{array}{lllll}21 & 20 & 20 & 21 & 20 \\ 22 & 22 & 21 & 23 & 21 \\ 25 & 24 & 24 & 25 & 24 \\ 31 & 28 & 29 & 30 & 31\end{array}$

Lithuania Poland

(1989-95) (1986-91)

$0 \quad 0$

$2 \quad 2$

$13 \quad 12$

$38 \quad 32$

$61 \quad 52$

$67 \quad 59$

$74 \quad 66$

$79 \quad 75$

$84 \quad 79$

$88 \quad 83$

$89 \quad 85$

$23 \quad 24$

$20 \quad 20$

$21 \quad 22$

$23 \quad 24$

$27 \quad 28$ 
Table 11: Cumulative percent ever "parent and not in a union" / Men

$\begin{array}{lcccc}\text { age } & \begin{array}{c}\text { Sweden } \\ (1978-93)\end{array} & \begin{array}{c}\text { Norway } \\ (1974-89)\end{array} & \begin{array}{c}\text { Finland } \\ (1983-92)\end{array} & \begin{array}{c}\text { France } \\ (1988-94)\end{array} \\ 16 & 0 & 0 & 0 & 0 \\ 18 & 0 & 0 & 0 & 0 \\ 20 & 0 & 1 & 0 & 1 \\ 22 & 1 & 2 & 1 & 1 \\ 24 & 2 & 4 & 2 & 2 \\ 25 & 3 & 4 & 2 & 2 \\ 26 & 4 & 5 & 3 & 2 \\ 28 & 8 & 8 & 4 & 4 \\ 30 & 10 & 10 & 6 & 6 \\ 35 & 16 & 15 & 9 & 11 \\ 40 & 22 & 20 & 14 & 15 \\ & & & & \\ \text { mean age: } & 31 & 30 & 31 & 31 \\ \text { (at transition) } & & & & \\ \text { 1st decile at age: } & 30 & 30 & 36 & 34 \\ \text { 1st quartile at: } & -- & -- & -- & -- \\ \text { median at age: } & -- & -- & -- & -- \\ \text { 3rd quartile at: } & -- & -- & -- & --\end{array}$

age

16

18

20

22

24

25

26

28

30

35

40

mean age:

(at transition)

1st decile at age:

1st quartile at:

median at age:

3rd quartile at:
West

Austria Germany Flanders Italy Spain

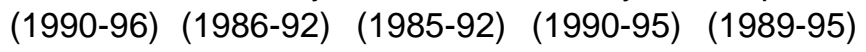

$\begin{array}{ccccc}0 & 0 & 0 & 0 & 0 \\ 0 & 0 & 0 & 0 & 1 \\ 1 & 0 & 1 & 0 & 1 \\ 3 & 1 & 1 & 0 & 2 \\ 5 & 1 & 1 & 0 & 2 \\ 6 & 2 & 1 & 0 & 2 \\ 7 & 2 & 2 & 1 & 3 \\ 9 & 3 & 3 & 1 & 3 \\ 11 & 4 & 3 & 1 & 3 \\ 14 & 9 & 7 & 2 & 5 \\ 17 & 13 & 11 & 4 & 6 \\ 28 & 32 & 32 & 32 & 29 \\ & & & & \\ 29 & 36 & 38 & -- & -- \\ -- & -- & -- & -- & -- \\ -- & -- & -- & -- & -- \\ -- & -- & -- & -- & -\end{array}$


Table 11: Cumulative percent ever "parent and not in a union" / Men

\begin{tabular}{|c|c|c|c|c|c|}
\hline age & $\begin{array}{c}\text { GDR } \\
(1984-89)\end{array}$ & $\begin{array}{c}\text { Hungary } \\
(1988-93)\end{array}$ & $\begin{array}{c}\text { Czech R } \\
\text { (NA) }\end{array}$ & $\begin{array}{c}\text { Slovenia } \\
(1989-95)\end{array}$ & $\begin{array}{l}\text { Latvia } \\
(1989-95)\end{array}$ \\
\hline 16 & 0 & 0 & & 0 & 0 \\
\hline 18 & 1 & 0 & & 0 & 0 \\
\hline 20 & 3 & 0 & & 0 & 1 \\
\hline 22 & 8 & 1 & & 1 & 3 \\
\hline 24 & 12 & 3 & & 3 & 6 \\
\hline 25 & 16 & 3 & & 4 & 8 \\
\hline 26 & 18 & 4 & & 5 & 10 \\
\hline 28 & 20 & 4 & & 6 & 12 \\
\hline 30 & 23 & 7 & & 8 & 15 \\
\hline 35 & 29 & 10 & & 10 & 21 \\
\hline 40 & -- & 14 & & 12 & 24 \\
\hline $\begin{array}{l}\text { mean age: } \\
\text { (at transition) }\end{array}$ & 27 & 30 & & 28 & 28 \\
\hline 1st decile at age: & 24 & 35 & & 33 & 26 \\
\hline 1st quartile at: & 32 & -- & & -- & -- \\
\hline median at age: & -- & -- & & -- & -- \\
\hline 3rd quartile at: & -- & -- & & -- & -- \\
\hline
\end{tabular}

$\begin{array}{ccc}\text { Lithuania } & \text { Poland } \\ (1989-95) & \text { (NA) }\end{array}$

$\begin{array}{lc}16 & 0 \\ 18 & 0 \\ 20 & 1 \\ 22 & 1 \\ 24 & 4 \\ 25 & 5 \\ 26 & 7 \\ 28 & 8 \\ 30 & 11 \\ 35 & 15 \\ 40 & 19 \\ & \\ \text { mean age: } & \\ \text { (at transition) } & 29 \\ & \\ \text { 1st decile at age: } & \\ \text { 1st quartile at: } & 29 \\ \text { median at age: } & -- \\ \text { 3rd quartile at: } & -- \\ \end{array}$


Table 11: Cumulative percent ever "parent and not in a union" / Women

$\begin{array}{lccccc}\text { age } & \begin{array}{c}\text { Sweden } \\ (1978-93)\end{array} & \begin{array}{c}\text { Norway } \\ (1974-89)\end{array} & \begin{array}{c}\text { Finland } \\ (1979-89)\end{array} & \begin{array}{c}\text { France } \\ (1988-94)\end{array} & \begin{array}{c}\text { USA } \\ (1989-95)\end{array} \\ 16 & 0 & 0 & 0 & 0 & 1 \\ 18 & 0 & 1 & 1 & 1 & 6 \\ 20 & 2 & 4 & 2 & 2 & 12 \\ 22 & 4 & 7 & 3 & 4 & 19 \\ 24 & 7 & 10 & 5 & 6 & 24 \\ 25 & 8 & 11 & 6 & 8 & 26 \\ 26 & 10 & 12 & 7 & 8 & 28 \\ 28 & 12 & 14 & 9 & 11 & 31 \\ 30 & 15 & 16 & 12 & 14 & 33 \\ 35 & 21 & 21 & 17 & 20 & 39 \\ 40 & 27 & 27 & 22 & 25 & 44 \\ & & & & & \\ \text { mean age: } & 29 & 28 & 29 & 29 & 25 \\ \text { (at transition) } & & & & & \\ & & & & & \\ \text { 1st decile at age: } & 26 & 24 & 29 & 28 & 20 \\ \text { 1st quartile at: } & 38 & 39 & -- & 40 & 25 \\ \text { median at age: } & -- & -- & -- & -- & -- \\ \text { 3rd quartile at: } & -- & -- & -- & -- & --\end{array}$

$\begin{array}{ccccc}\text { Austria } & \text { Germany } & \text { Flanders } & \text { Italy } & \text { Spain } \\ (1990-96) & (1986-92) & (1985-92) & (1990-95) & (1989-95)\end{array}$

$\begin{array}{lccccc}16 & 0 & 0 & 0 & 0 & 0 \\ 18 & 1 & 0 & 0 & 1 & 1 \\ 20 & 5 & 1 & 1 & 1 & 2 \\ 22 & 9 & 2 & 1 & 1 & 3 \\ 24 & 13 & 4 & 2 & 1 & 4 \\ 25 & 15 & 5 & 2 & 1 & 4 \\ 26 & 17 & 6 & 3 & 1 & 5 \\ 28 & 21 & 9 & 4 & 2 & 6 \\ 30 & 25 & 12 & 6 & 3 & 7 \\ 35 & 31 & 17 & 10 & 6 & 10 \\ 40 & 35 & 21 & 15 & 8 & 12 \\ & & & & & \\ \text { mean age: } & 27 & 29 & 32 & 30 & 28 \\ \text { (at transition) } & & & & & \\ \text { 1st decile at age: } & 23 & 29 & 35 & -- & 35 \\ \text { 1st quartile at: } & 30 & -- & -- & -- & -- \\ \text { median at age: } & -- & -- & -- & -- & -- \\ \text { 3rd quartile at: } & -- & -- & -- & -- & -\end{array}$


Table 11: Cumulative percent ever "parent and not in a union" / Women

$\begin{array}{lccccc}\text { age } & \begin{array}{c}\text { GDR } \\ (1984-89)\end{array} & \begin{array}{c}\text { Hungary } \\ (1988-93)\end{array} & \begin{array}{c}\text { Czech R } \\ (1992-97)\end{array} & \begin{array}{c}\text { Slovenia } \\ (1989-95)\end{array} & \begin{array}{c}\text { Latvia } \\ (1989-95)\end{array} \\ 16 & & & & & \\ 18 & 0 & 0 & 0 & 0 & 0 \\ 20 & 2 & 1 & 1 & 1 & 2 \\ 22 & 8 & 3 & 4 & 3 & 6 \\ 24 & 18 & 5 & 9 & 6 & 12 \\ 25 & 24 & 8 & 13 & 10 & 18 \\ 26 & 27 & 9 & 16 & 10 & 22 \\ 28 & 29 & 10 & 17 & 11 & 25 \\ 30 & 34 & 13 & 21 & 13 & 30 \\ 35 & 38 & 16 & 23 & 15 & 34 \\ 40 & 45 & 22 & 29 & 17 & 44 \\ & -- & 26 & 35 & 20 & 49 \\ \text { mean age: } & & & & & \\ \text { (at transition) } & 25 & 28 & 27 & 26 & 27 \\ & & & & & \\ \text { 1st decile at age: } & 21 & 26 & 23 & 24 & 22 \\ \text { 1st quartile at: } & 25 & 39 & 32 & -- & 26 \\ \text { median at age: } & -- & -- & -- & -- & - \\ \text { 3rd quartile at: } & -- & -- & -- & -- & -\end{array}$

$\begin{array}{ccc}\text { age } & \text { Lithuania } & \text { Poland } \\ (1989-95) & (1986-91)\end{array}$

$\begin{array}{lcc}16 & 0 & 0 \\ 18 & 0 & 1 \\ 20 & 2 & 4 \\ 22 & 4 & 7 \\ 24 & 8 & 9 \\ 25 & 10 & 10 \\ 26 & 12 & 11 \\ 28 & 15 & 13 \\ 30 & 17 & 14 \\ 35 & 24 & 18 \\ 40 & 29 & 21 \\ & & \\ \text { mean age: } & & \\ \text { (at transition) } & 28 & 27 \\ & & \\ \text { 1st decile at age: } & & \\ \text { 1st quartile at: } & 25 & 25 \\ \text { median at age: } & 36 & -- \\ \text { 3rd quartile at: } & -- & -- \\ & -- & --\end{array}$


Table 12: Cumulative percent ever "married ever during a first union" (including those who marry directly) / Men

$\begin{array}{cccccc}\text { age } & \text { Sweden } & \text { Norway } & \text { Finland } & \text { France } & \text { USA } \\ (1978-93) & (1974-89) & (1983-92) & (1988-94) & \text { (NA) }\end{array}$

$\begin{array}{lcccc}16 & 0 & 0 & 0 & 0 \\ 18 & 0 & 0 & 0 & 1 \\ 20 & 1 & 3 & 1 & 1 \\ 22 & 2 & 11 & 5 & 5 \\ 24 & 7 & 24 & 13 & 11 \\ 25 & 10 & 30 & 17 & 15 \\ 26 & 15 & 36 & 22 & 22 \\ 28 & 21 & 46 & 35 & 31 \\ 30 & 29 & 58 & 46 & 40 \\ 35 & 43 & 71 & 58 & 47 \\ 40 & 48 & 75 & 62 & 52 \\ & & & & \\ \text { mean age: } & 29 & 27 & 28 & 28 \\ \text { (at transition) } & & & & \\ & & & & \\ \text { 1st decile at age: } & 25 & 22 & 24 & 24 \\ \text { 1st quartile at: } & 29 & 25 & 27 & 27 \\ \text { median at age: } & -- & 29 & 32 & 38 \\ \text { 3rd quartile at: } & -- & 40 & -- & --\end{array}$

age

16

18

20

22

24

25

26

28

30

35

40

mean age:

(at transition)

1st decile at age:

1st quartile at:

median at age:

3rd quartile at:
West

Austria Germany Flanders Italy Spain (1990-96) (1986-92) (1985-92) $\quad(1990-95) \quad(1989-95)$

$\begin{array}{ccccc}0 & 0 & 0 & 0 & 0 \\ 0 & 0 & 0 & 0 & 0 \\ 2 & 1 & 0 & 0 & 2 \\ 5 & 4 & 7 & 1 & 6 \\ 13 & 8 & 26 & 6 & 13 \\ 18 & 11 & 36 & 10 & 19 \\ 23 & 14 & 47 & 13 & 25 \\ 31 & 20 & 58 & 27 & 40 \\ 42 & 28 & 66 & 38 & 50 \\ 48 & 38 & 73 & 56 & 68 \\ 50 & 41 & -- & 69 & 73\end{array}$

$\begin{array}{lllll}27 & 28 & 26 & 30 & 28\end{array}$

$\begin{array}{lllll}23 & 25 & 23 & 25 & 23\end{array}$

$\begin{array}{lllll}27 & 29 & 24 & 28 & 26\end{array}$

$\begin{array}{lllll}37 & -- & 27 & 33 & 30\end{array}$


Table 12: Cumulative percent ever "married ever during a first union" (including those who marry directly) / Men

\begin{tabular}{|c|c|c|c|c|c|}
\hline age & $\begin{array}{c}\text { GDR } \\
(1984-89)\end{array}$ & $\begin{array}{l}\text { Hungary } \\
(1988-93)\end{array}$ & $\begin{array}{c}\text { Czech R } \\
\text { (NA) }\end{array}$ & $\begin{array}{c}\text { Slovenia } \\
(1989-95)\end{array}$ & $\begin{array}{c}\text { Latvia } \\
(1989-95)\end{array}$ \\
\hline 16 & 0 & 0 & & 0 & 0 \\
\hline 18 & 0 & 0 & & 0 & 0 \\
\hline 20 & 2 & 4 & & 0 & 8 \\
\hline 22 & 14 & 15 & & 6 & 25 \\
\hline 24 & 32 & 34 & & 23 & 46 \\
\hline 25 & 42 & 42 & & 31 & 53 \\
\hline 26 & 50 & 48 & & 37 & 60 \\
\hline 28 & 60 & 59 & & 54 & 67 \\
\hline 30 & 67 & 64 & & 65 & 71 \\
\hline 35 & 73 & 69 & & 76 & 74 \\
\hline 40 & -- & 71 & & 82 & 75 \\
\hline $\begin{array}{l}\text { mean age: } \\
\text { (at transition) }\end{array}$ & 25 & 25 & & 27 & 24 \\
\hline 1st decile at age: & 22 & 22 & & 23 & 21 \\
\hline 1st quartile at: & 24 & 23 & & 25 & 22 \\
\hline median at age: & 26 & 27 & & 28 & 25 \\
\hline 3rd quartile at: & -- & -- & & 34 & 36 \\
\hline
\end{tabular}

$\begin{array}{cc}\text { Lithuania } & \text { Poland } \\ (1989-95) & \text { (NA) }\end{array}$

$16-0$

$18-1$

$20 \quad 9$

$22 \quad 29$

$24 \quad 54$

$25 \quad 63$

$26 \quad 73$

$28 \quad 82$

$30 \quad 88$

$35 \quad 93$

$40 \quad 94$

mean age: $\quad 24$

(at transition)

1st decile at age: $\quad 21$

1st quartile at: $\quad 22$

median at age: $\quad 24$

3rd quartile at: $\quad 27$ 
Table 12: Cumulative percent ever "married ever during a first union" (including those who marry directly) / Women

age

16

18

20

22

24

25

26

28

30

35

40

mean age:

(at transition)

1st decile at age:

1st quartile at:

median at age:

$3 r d$ quartile at:

age

16

18

20

22

24

25

26

28

30

35

40

mean age:

(at transition)

1st decile at age:

1st quartile at:

median at age:

3rd quartile at:
Sweden Norway Finland France USA $\begin{array}{lllll}(1978-93) & (1974-89) & (1979-89) & (1988-94) & (1989-95)\end{array}$

$\begin{array}{ccccc}0 & 0 & 0 & 0 & 0 \\ 0 & 2 & 2 & 1 & 4 \\ 2 & 11 & 8 & 7 & 15 \\ 7 & 27 & 20 & 13 & 28 \\ 14 & 41 & 34 & 21 & 41 \\ 18 & 48 & 40 & 27 & 45 \\ 24 & 53 & 46 & 33 & 49 \\ 31 & 61 & 55 & 43 & 57 \\ 36 & 67 & 61 & 45 & 62 \\ 45 & 74 & 69 & 55 & 69 \\ 53 & 76 & 72 & 58 & 71 \\ & & & & \\ 28 & 24 & 25 & 26 & 24 \\ & & & & \\ & & & & \\ 23 & 20 & 21 & 22 & 20 \\ 27 & 22 & 23 & 25 & 22 \\ 36 & 26 & 27 & 33 & 27 \\ -- & 36 & -- & -- & --\end{array}$

West

Austria Germany Flanders Italy Spain (1990-96) (1986-92) (1985-92) $\quad(1990-95) \quad(1989-95)$

$\begin{array}{ccccc}0 & 0 & 0 & 0 & 0 \\ 2 & 2 & 1 & 0 & 2 \\ 7 & 5 & 8 & 4 & 8 \\ 16 & 13 & 26 & 11 & 17 \\ 31 & 21 & 48 & 21 & 29 \\ 35 & 26 & 58 & 27 & 38 \\ 41 & 30 & 64 & 36 & 45 \\ 54 & 37 & 74 & 49 & 61 \\ 60 & 42 & 79 & 58 & 70 \\ 68 & 49 & 83 & 75 & 81 \\ 70 & 51 & -- & 80 & 83\end{array}$

$25 \quad 25$

$24 \quad 27$

26

$\begin{array}{lllll}21 & 22 & 21 & 22 & 21 \\ 24 & 25 & 22 & 25 & 24 \\ 28 & 36 & 25 & 29 & 27 \\ -- & -- & 29 & 35 & 33\end{array}$


Table 12: Cumulative percent ever "married ever during a first union" (including those who marry directly) / Women

age

16

18

20

22

24

25

26

28

30

35

40

mean age:

(at transition)

1st decile at age:

1st quartile at:

median at age:

3rd quartile at:

age

16

18

20

22

24

25

26

28

30

35

40

mean age:

(at transition)

1st decile at age:

1st quartile at:

median at age:

3rd quartile at:
GDR Hungary Czech R Slovenia Latvia $\begin{array}{lllll}(1984-89) & (1988-93) & (1992-97) & (1989-95) & (1989-95)\end{array}$

$\begin{array}{lllll}0 & 0 & 0 & 0 & 0\end{array}$

$\begin{array}{lllll}1 & 5 & 3 & 1 & 5\end{array}$

$14 \quad 26$

$37 \quad 48$

$56 \quad 66$

$61 \quad 72$

$64 \quad 76$

$70 \quad 80$

$73 \quad 81$

$76 \quad 84$

$--\quad 86$

$23 \quad 22$

$22 \quad 23$

11

31

29

$\begin{array}{lll}22 & 11 & 29 \\ 43 & 31 & 48\end{array}$

$\begin{array}{lll}60 & 49 & 58\end{array}$

$\begin{array}{lll}69 & 58 & 62\end{array}$

$\begin{array}{lll}72 & 63 & 67\end{array}$

$\begin{array}{lll}79 & 73 & 72\end{array}$

$\begin{array}{lll}83 & 77 & 76\end{array}$

$85 \quad 82 \quad 78$

$86 \quad 82 \quad 80$

$23 \quad 24 \quad 22$

$\begin{array}{lllll}20 & 19 & 20 & 20 & 19\end{array}$

$\begin{array}{lllll}21 & 20 & 21 & 22 & 20\end{array}$

$\begin{array}{lllll}24 & 23 & 23 & 25 & 23\end{array}$

$\begin{array}{lllll}31 & 26 & 27 & 29 & 30\end{array}$

Lithuania Poland

(1989-95) (1986-91)

$0 \quad 0$

$5 \quad 4$

$29 \quad 21$

$55 \quad 46$

$72 \quad 65$

$77 \quad 72$

$81 \quad 76$

$87 \quad 83$

$90 \quad 85$

$93 \quad 88$

$93 \quad 90$

$22 \quad 23$

$19 \quad 19$

$20 \quad 21$

$22 \quad 23$

$25 \quad 26$ 
Table 13: Cumulative percent ever "having a first birth ever in a first union" / Men

$\begin{array}{lcccc}\text { age } & \begin{array}{c}\text { Sweden } \\ (1978-93)\end{array} & \begin{array}{c}\text { Norway } \\ (1974-89)\end{array} & \begin{array}{c}\text { Finland } \\ (1983-92)\end{array} & \begin{array}{c}\text { France } \\ (1988-94)\end{array} \\ 16 & 0 & 0 & 0 & 0 \\ 18 & 0 & 0 & 0 & 0 \\ 20 & 1 & 2 & 1 & 0 \\ 22 & 5 & 7 & 3 & 3 \\ 24 & 13 & 16 & 8 & 7 \\ 25 & 17 & 23 & 13 & 10 \\ 26 & 21 & 29 & 16 & 14 \\ 28 & 31 & 41 & 26 & 30 \\ 30 & 42 & 53 & 39 & 43 \\ 35 & 55 & 67 & 55 & 56 \\ 40 & 57 & 70 & 61 & 62 \\ & & & & \\ \text { mean age: } & 28 & 27 & 29 & 29 \\ \text { (at transition) } & & & & \\ & & & & \\ \text { 1st decile at age: } & 24 & 23 & 25 & 25 \\ \text { 1st quartile at: } & 27 & 26 & 28 & 28 \\ \text { median at age: } & 33 & 30 & 32 & 32 \\ \text { 3rd quartile at: } & -- & -- & -- & -\end{array}$

age

16

18

20

22

24

25

26

28

30

35

40

mean age:

(at transition)

1st decile at age:

1st quartile at:

median at age:

3rd quartile at:
West

Austria Germany Flanders Italy Spain

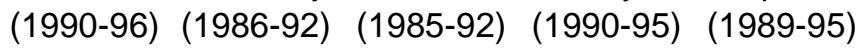

$\begin{array}{ccccc}0 & 0 & 0 & 0 & 0 \\ 0 & 0 & 0 & 0 & 0 \\ 0 & 1 & 0 & 0 & 1 \\ 2 & 2 & 2 & 0 & 3 \\ 6 & 4 & 8 & 3 & 7 \\ 11 & 6 & 13 & 4 & 10 \\ 16 & 8 & 21 & 7 & 15 \\ 24 & 14 & 39 & 14 & 24 \\ 33 & 21 & 53 & 25 & 38 \\ 44 & 34 & 69 & 49 & 61 \\ 50 & 37 & 73 & 58 & 69 \\ 29 & 29 & 28 & 31 & 30\end{array}$

$\begin{array}{lllll}25 & 27 & 25 & 28 & 25 \\ 29 & 31 & 27 & 30 & 29 \\ 38 & -- & 30 & 36 & 33 \\ -- & -- & -- & -- & --\end{array}$


Table 13: Cumulative percent ever "having a first birth ever in a first union" / Men

\begin{tabular}{|c|c|c|c|c|c|}
\hline age & $\begin{array}{c}\text { GDR } \\
(1984-89)\end{array}$ & $\begin{array}{l}\text { Hungary } \\
(1988-93)\end{array}$ & $\begin{array}{c}\text { Czech R } \\
\text { (NA) }\end{array}$ & $\begin{array}{c}\text { Slovenia } \\
(1989-95)\end{array}$ & $\begin{array}{c}\text { Latvia } \\
(1989-95)\end{array}$ \\
\hline 16 & 0 & 0 & & 0 & 0 \\
\hline 18 & 1 & 0 & & 0 & 0 \\
\hline 20 & 1 & 3 & & 1 & 5 \\
\hline 22 & 9 & 8 & & 4 & 15 \\
\hline 24 & 21 & 23 & & 24 & 34 \\
\hline 25 & 27 & 31 & & 33 & 44 \\
\hline 26 & 34 & 37 & & 42 & 51 \\
\hline 28 & 46 & 50 & & 55 & 63 \\
\hline 30 & 52 & 55 & & 67 & 68 \\
\hline 35 & 60 & 65 & & 77 & 71 \\
\hline 40 & -- & 68 & & 80 & 73 \\
\hline $\begin{array}{l}\text { mean age: } \\
\text { (at transition) }\end{array}$ & 26 & 26 & & 26 & 25 \\
\hline 1st decile at age: & 23 & 23 & & 23 & 21 \\
\hline 1st quartile at: & 25 & 25 & & 25 & 24 \\
\hline median at age: & 30 & 28 & & 28 & 26 \\
\hline 3rd quartile at: & -- & -- & & 33 & -- \\
\hline
\end{tabular}

$\begin{array}{ccc}\text { Lithuania } & \text { Poland } \\ (1989-95) & \text { (NA) }\end{array}$

$\begin{array}{lc}16 & 0 \\ 18 & 0 \\ 20 & 4 \\ 22 & 14 \\ 24 & 35 \\ 25 & 43 \\ 26 & 52 \\ 28 & 66 \\ 30 & 76 \\ 35 & 81 \\ 40 & 83 \\ & \\ \text { mean age: } & 25 \\ \text { (at transition) } & \\ & \\ \text { 1st decile at age: } & 22 \\ \text { 1st quartile at: } & 24 \\ \text { median at age: } & 26 \\ \text { 3rd quartile at: } & 30\end{array}$


Table 13: Cumulative percent ever "having a first birth ever in a first union" / Women

\begin{tabular}{|c|c|c|c|c|c|}
\hline age & $\begin{array}{c}\text { Sweden } \\
(1978-93)\end{array}$ & $\begin{array}{c}\text { Norway } \\
(1974-89)\end{array}$ & $\begin{array}{c}\text { Finland } \\
(1979-89)\end{array}$ & $\begin{array}{c}\text { France } \\
(1988-94)\end{array}$ & $\begin{array}{c}\text { USA } \\
(1989-95)\end{array}$ \\
\hline 16 & 0 & 0 & 0 & 0 & 0 \\
\hline 18 & 1 & 1 & 2 & 1 & 3 \\
\hline 20 & 5 & 8 & 7 & 5 & 10 \\
\hline 22 & 12 & 21 & 16 & 10 & 17 \\
\hline 24 & 23 & 33 & 25 & 21 & 24 \\
\hline 25 & 28 & 39 & 32 & 27 & 27 \\
\hline 26 & 33 & 44 & 38 & 33 & 30 \\
\hline 28 & 44 & 55 & 50 & 45 & 37 \\
\hline 30 & 52 & 62 & 58 & 53 & 42 \\
\hline 35 & 60 & 68 & 68 & 64 & 51 \\
\hline 40 & 62 & 70 & 71 & 67 & 53 \\
\hline $\begin{array}{l}\text { mean age: } \\
\text { (at transition) }\end{array}$ & 26 & 25 & 26 & 27 & 25 \\
\hline 1st decile at age: & 22 & 21 & 21 & 22 & 20 \\
\hline 1st quartile at: & 25 & 23 & 24 & 25 & 25 \\
\hline median at age: & 30 & 27 & 28 & 30 & 34 \\
\hline 3rd quartile at: & -- & -- & -- & -- & -- \\
\hline age & $\begin{array}{c}\text { Austria } \\
(1990-96)\end{array}$ & $\begin{array}{c}\text { West } \\
\text { Germany } \\
(1986-92)\end{array}$ & $\begin{array}{c}\text { Flanders } \\
\text { (1985-92) }\end{array}$ & $\begin{array}{c}\text { Italy } \\
(1990-95)\end{array}$ & $\begin{array}{c}\text { Spain } \\
(1989-95)\end{array}$ \\
\hline
\end{tabular}

$\begin{array}{lccccc}16 & 0 & 0 & 0 & 1 & 0 \\ 18 & 1 & 0 & 0 & 2 & 1 \\ 20 & 7 & 2 & 2 & 3 & 6 \\ 22 & 16 & 7 & 7 & 7 & 12 \\ 24 & 30 & 14 & 18 & 13 & 20 \\ 25 & 36 & 17 & 26 & 18 & 24 \\ 26 & 43 & 23 & 36 & 24 & 29 \\ 28 & 55 & 32 & 54 & 35 & 43 \\ 30 & 61 & 40 & 66 & 48 & 57 \\ 35 & 67 & 49 & 74 & 68 & 75 \\ 40 & 69 & 50 & 75 & 74 & 79 \\ & & & & & \\ \text { mean age: } & 25 & 27 & 26 & 28 & 27 \\ \text { (at transition) } & & & & & \\ \text { 1st decile at age: } & 21 & 23 & 23 & 23 & 22 \\ \text { 1st quartile at: } & 24 & 27 & 25 & 27 & 26 \\ \text { median at age: } & 27 & 36 & 28 & 31 & 30 \\ \text { 3rd quartile at: } & -- & -- & 39 & -- & 35\end{array}$


Table 13: Cumulative percent ever "having a first birth ever in a first union" / Women

\begin{tabular}{|c|c|c|c|c|c|}
\hline age & $\begin{array}{c}\text { GDR } \\
(1984-89)\end{array}$ & $\begin{array}{c}\text { Hungary } \\
(1988-93)\end{array}$ & $\begin{array}{c}\text { Czech R } \\
(1992-97)\end{array}$ & $\begin{array}{c}\text { Slovenia } \\
(1989-95)\end{array}$ & $\begin{array}{c}\text { Latvia } \\
(1989-95)\end{array}$ \\
\hline 16 & 0 & 0 & 0 & 0 & 0 \\
\hline 18 & 1 & 3 & 2 & 1 & 3 \\
\hline 20 & 7 & 15 & 16 & 9 & 17 \\
\hline 22 & 24 & 34 & 34 & 27 & 37 \\
\hline 24 & 40 & 52 & 47 & 48 & 50 \\
\hline 25 & 46 & 59 & 54 & 55 & 55 \\
\hline 26 & 51 & 65 & 60 & 63 & 59 \\
\hline 28 & 55 & 74 & 70 & 71 & 67 \\
\hline 30 & 58 & 76 & 74 & 75 & 70 \\
\hline 35 & 61 & 81 & 78 & 79 & 74 \\
\hline 40 & -- & 82 & 79 & 80 & 75 \\
\hline $\begin{array}{l}\text { mean age: } \\
\text { (at transition) }\end{array}$ & 23 & 23 & 23 & 24 & 23 \\
\hline 1st decile at age: & 21 & 20 & 20 & 21 & 20 \\
\hline 1st quartile at: & 23 & 22 & 21 & 22 & 21 \\
\hline median at age: & 26 & 24 & 25 & 25 & 24 \\
\hline 3rd quartile at: & -- & 29 & 31 & 30 & 37 \\
\hline age & $\begin{array}{l}\text { Lithuania } \\
(1989-95)\end{array}$ & $\begin{array}{l}\text { Poland } \\
(1986-91)\end{array}$ & & & \\
\hline 16 & 0 & 0 & & & \\
\hline 18 & 2 & 1 & & & \\
\hline 20 & 13 & 11 & & & \\
\hline 22 & 38 & 31 & & & \\
\hline 24 & 59 & 50 & & & \\
\hline 25 & 65 & 57 & & & \\
\hline 26 & 71 & 63 & & & \\
\hline 28 & 76 & 72 & & & \\
\hline 30 & 79 & 75 & & & \\
\hline 35 & 83 & 79 & & & \\
\hline 40 & 84 & 80 & & & \\
\hline $\begin{array}{l}\text { mean age: } \\
\text { (at transition) }\end{array}$ & 23 & 24 & & & \\
\hline 1st decile at age: & 20 & 20 & & & \\
\hline 1st quartile at: & 21 & 22 & & & \\
\hline median at age: & 23 & 24 & & & \\
\hline 3rd quartile at: & 28 & 30 & & & \\
\hline
\end{tabular}


Table 14: Cumulative percent ever "having a first birth ever in any union" / Men

$\begin{array}{lcccc}\text { age } & \begin{array}{c}\text { Sweden } \\ (1978-93)\end{array} & \begin{array}{c}\text { Norway } \\ (1974-89)\end{array} & \begin{array}{c}\text { Finland } \\ (1983-92)\end{array} & \begin{array}{c}\text { France } \\ (1988-94)\end{array} \\ 16 & 0 & 0 & 0 & 0 \\ 18 & 0 & 0 & 0 & 0 \\ 20 & 1 & 2 & 1 & 0 \\ 22 & 5 & 7 & 3 & 3 \\ 24 & 14 & 16 & 9 & 8 \\ 25 & 19 & 24 & 14 & 10 \\ 26 & 25 & 31 & 17 & 16 \\ 28 & 38 & 44 & 29 & 34 \\ 30 & 51 & 56 & 43 & 49 \\ 35 & 69 & 70 & 62 & 66 \\ 40 & 73 & 74 & 68 & 74 \\ & & & & \\ \text { mean age: } & 28 & 27 & 29 & 29 \\ \text { (at transition) } & & & & \\ \text { 1st decile at age: } & 24 & 23 & 25 & 25 \\ \text { 1st quartile at: } & 26 & 26 & 28 & 27 \\ \text { median at age: } & 30 & 29 & 31 & 31 \\ \text { 3rd quartile at: } & -- & -- & -- & --\end{array}$

age

16

18

20

22

24

25

26

28

30

35

40

mean age:

(at transition)

1st decile at age:

1st quartile at:

median at age:

3rd quartile at:
West

Austria Germany Flanders Italy Spain

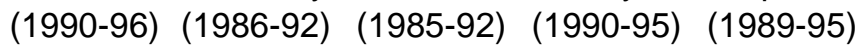

$\begin{array}{ccccc}0 & 0 & 0 & 0 & 0 \\ 0 & 0 & 0 & 0 & 0 \\ 1 & 1 & 0 & 0 & 1 \\ 3 & 2 & 2 & 0 & 3 \\ 7 & 4 & 8 & 3 & 8 \\ 12 & 6 & 14 & 4 & 10 \\ 17 & 8 & 21 & 7 & 15 \\ 26 & 14 & 40 & 14 & 25 \\ 38 & 24 & 55 & 26 & 39 \\ 53 & 39 & 72 & 50 & 64 \\ 63 & 43 & 77 & 60 & 72 \\ 29 & 30 & 28 & 31 & 30\end{array}$

$\begin{array}{ccccc}25 & 27 & 25 & 28 & 25 \\ 28 & 31 & 27 & 30 & 28 \\ 34 & -- & 30 & 35 & 32 \\ -- & -- & 40 & -- & --\end{array}$


Table 14: Cumulative percent ever "having a first birth ever in any union" / Men

\begin{tabular}{|c|c|c|c|c|c|}
\hline age & $\begin{array}{c}\text { GDR } \\
(1984-89)\end{array}$ & $\begin{array}{l}\text { Hungary } \\
(1988-93)\end{array}$ & $\begin{array}{c}\text { Czech R } \\
\text { (NA) }\end{array}$ & $\begin{array}{l}\text { Slovenia } \\
(1989-95)\end{array}$ & $\begin{array}{c}\text { Latvia } \\
(1989-95)\end{array}$ \\
\hline 16 & 0 & 0 & & 0 & 0 \\
\hline 18 & 1 & 0 & & 0 & 0 \\
\hline 20 & 1 & 3 & & 1 & 5 \\
\hline 22 & 9 & 8 & & 4 & 15 \\
\hline 24 & 22 & 23 & & 24 & 36 \\
\hline 25 & 28 & 32 & & 33 & 47 \\
\hline 26 & 35 & 39 & & 43 & 54 \\
\hline 28 & 48 & 52 & & 58 & 68 \\
\hline 30 & 55 & 61 & & 70 & 74 \\
\hline 35 & 66 & 73 & & 81 & 78 \\
\hline 40 & -- & 76 & & 85 & 79 \\
\hline $\begin{array}{l}\text { mean age: } \\
\text { (at transition) }\end{array}$ & 26 & 27 & & 27 & 25 \\
\hline 1st decile at age: & 23 & 23 & & 23 & 21 \\
\hline 1st quartile at: & 25 & 25 & & 25 & 23 \\
\hline median at age: & 29 & 28 & & 27 & 26 \\
\hline 3rd quartile at: & -- & 37 & & 31 & 32 \\
\hline
\end{tabular}

$\begin{array}{ccc}\text { Lithuania } & \text { Poland } \\ (1989-95) & \text { (NA) }\end{array}$

$\begin{array}{lc}16 & 0 \\ 18 & 0 \\ 20 & 4 \\ 22 & 14 \\ 24 & 35 \\ 25 & 44 \\ 26 & 52 \\ 28 & 66 \\ 30 & 76 \\ 35 & 82 \\ 40 & 84 \\ & \\ \text { mean age: } & 25 \\ \text { (at transition) } & \\ & \\ \text { 1st decile at age: } & 22 \\ \text { 1st quartile at: } & 24 \\ \text { median at age: } & 26 \\ \text { 3rd quartile at: } & 30\end{array}$


Table 14: Cumulative percent ever "having a first birth ever in any union" / Women

\begin{tabular}{|c|c|c|c|c|c|}
\hline age & $\begin{array}{l}\text { Sweden } \\
(1978-93)\end{array}$ & $\begin{array}{c}\text { Norway } \\
(1974-89)\end{array}$ & $\begin{array}{l}\text { Finland } \\
(1979-89)\end{array}$ & $\begin{array}{c}\text { France } \\
(1988-94)\end{array}$ & $\begin{array}{c}\text { USA } \\
(1989-95)\end{array}$ \\
\hline 16 & 0 & 0 & 0 & 0 & 0 \\
\hline 18 & 1 & 1 & 2 & 1 & 3 \\
\hline 20 & 5 & 8 & 7 & 5 & 10 \\
\hline 22 & 13 & 21 & 16 & 10 & 18 \\
\hline 24 & 27 & 34 & 26 & 22 & 27 \\
\hline 25 & 33 & 40 & 33 & 29 & 30 \\
\hline 26 & 39 & 46 & 40 & 35 & 34 \\
\hline 28 & 53 & 58 & 53 & 48 & 42 \\
\hline 30 & 63 & 65 & 63 & 58 & 50 \\
\hline 35 & 76 & 74 & 73 & 71 & 61 \\
\hline 40 & 79 & 76 & 77 & 75 & 65 \\
\hline $\begin{array}{l}\text { mean age: } \\
\text { (at transition) }\end{array}$ & 26 & 25 & 26 & 27 & 26 \\
\hline 1st decile at age: & 22 & 21 & 21 & 22 & 20 \\
\hline 1st quartile at: & 24 & 23 & 24 & 25 & 24 \\
\hline median at age: & 28 & 27 & 28 & 29 & 30 \\
\hline 3rd quartile at: & 35 & 37 & 36 & 38 & -- \\
\hline age & $\begin{array}{c}\text { Austria } \\
(1990-96)\end{array}$ & $\begin{array}{c}\text { West } \\
\text { Germany } \\
(1986-92)\end{array}$ & $\begin{array}{l}\text { Flanders } \\
\text { (1985-92) }\end{array}$ & $\begin{array}{c}\text { Italy } \\
(1990-95)\end{array}$ & $\begin{array}{c}\text { Spain } \\
(1989-95)\end{array}$ \\
\hline
\end{tabular}

$\begin{array}{lccccc}16 & 0 & 0 & 0 & 1 & 0 \\ 18 & 1 & 0 & 0 & 2 & 1 \\ 20 & 7 & 2 & 2 & 3 & 6 \\ 22 & 16 & 7 & 7 & 7 & 13 \\ 24 & 31 & 15 & 18 & 13 & 21 \\ 25 & 37 & 18 & 27 & 18 & 24 \\ 26 & 45 & 25 & 36 & 24 & 30 \\ 28 & 58 & 34 & 55 & 36 & 43 \\ 30 & 65 & 44 & 67 & 48 & 58 \\ 35 & 73 & 55 & 77 & 70 & 77 \\ 40 & 76 & 57 & 78 & 75 & 81 \\ & & & & & \\ \text { mean age: } & 25 & 27 & 27 & 28 & 27 \\ \text { (at transition) } & & & & & \\ & & & & & \\ \text { 1st decile at age: } & 21 & 23 & 23 & 23 & 21 \\ \text { 1st quartile at: } & 24 & 26 & 25 & 27 & 26 \\ \text { median at age: } & 27 & 32 & 28 & 31 & 30 \\ \text { 3rd quartile at: } & 37 & -- & 34 & 39 & 34\end{array}$


Table 14: Cumulative percent ever "having a first birth ever in any union" / Women

$\begin{array}{lccccc}\text { age } & \begin{array}{c}\text { GDR } \\ (1984-89)\end{array} & \begin{array}{c}\text { Hungary } \\ (1988-93)\end{array} & \begin{array}{c}\text { Czech R } \\ (1992-97)\end{array} & \begin{array}{c}\text { Slovenia } \\ (1989-95)\end{array} & \begin{array}{c}\text { Latvia } \\ (1989-95)\end{array} \\ 16 & 0 & 0 & 0 & 0 & 0 \\ 18 & 1 & 3 & 2 & 1 & 3 \\ 20 & 7 & 15 & 16 & 9 & 17 \\ 22 & 25 & 35 & 34 & 27 & 38 \\ 24 & 42 & 53 & 48 & 48 & 53 \\ 25 & 47 & 60 & 55 & 55 & 58 \\ 26 & 53 & 67 & 62 & 63 & 62 \\ 28 & 57 & 76 & 72 & 72 & 71 \\ 30 & 61 & 80 & 77 & 77 & 75 \\ 35 & 64 & 86 & 81 & 81 & 79 \\ 40 & -- & 87 & 83 & 82 & 80 \\ & & & & & \\ \text { mean age: } & 23 & 24 & 24 & 24 & 23 \\ \text { (at transition) } & & & & & \\ & & & & & \\ \text { 1st decile at age: } & 21 & 20 & 20 & 21 & 19 \\ \text { 1st quartile at: } & 22 & 21 & 21 & 22 & 21 \\ \text { median at age: } & 26 & 24 & 25 & 25 & 24 \\ \text { 3rd quartile at: } & -- & 28 & 29 & 29 & 30\end{array}$

$\begin{array}{llc}\text { age } & \text { Lithuania } & \text { Poland } \\ (1989-95) & (1986-91)\end{array}$

$\begin{array}{lcc}16 & 0 & 0 \\ 18 & 2 & 1 \\ 20 & 13 & 11 \\ 22 & 38 & 31 \\ 24 & 60 & 51 \\ 25 & 66 & 57 \\ 26 & 72 & 63 \\ 28 & 77 & 72 \\ 30 & 81 & 76 \\ 35 & 85 & 79 \\ 40 & 86 & 81 \\ & & \\ \text { mean age: } & 23 & 24 \\ \text { (at transition) } & & \\ & & \\ \text { 1st decile at age: } & 20 & 20 \\ \text { 1st quartile at: } & 21 & 22 \\ \text { median at age: } & 23 & 24 \\ \text { 3rd quartile at: } & 27 & 30\end{array}$


Table 15: Cumulative percent ever "having a first birth ever in any marriage" / Men

$\begin{array}{lcccc}\text { age } & \begin{array}{c}\text { Sweden } \\ (1978-93)\end{array} & \begin{array}{c}\text { Norway } \\ (1974-89)\end{array} & \begin{array}{c}\text { Finland } \\ (1983-92)\end{array} & \begin{array}{c}\text { France } \\ (1988-94)\end{array} \\ 16 & 0 & 0 & 0 & 0 \\ 18 & 0 & 0 & 0 & 0 \\ 20 & 0 & 1 & 0 & 0 \\ 22 & 1 & 5 & 2 & 2 \\ 24 & 3 & 10 & 5 & 4 \\ 25 & 4 & 16 & 9 & 6 \\ 26 & 5 & 20 & 10 & 9 \\ 28 & 9 & 29 & 18 & 22 \\ 30 & 14 & 42 & 30 & 32 \\ 35 & 23 & 57 & 47 & 43 \\ 40 & 26 & 62 & 53 & 50 \\ & & & & \\ \text { mean age: } & 30 & 28 & 29 & 29 \\ \text { (at transition) } & & & & \\ & & & & \\ \text { 1st decile at age: } & 29 & 24 & 26 & 27 \\ \text { 1st quartile at: } & 37 & 28 & 29 & 29 \\ \text { median at age: } & -- & 33 & 37 & 38 \\ \text { 3rd quartile at: } & -- & -- & -- & --\end{array}$

age

16

18

20

22

24

25

26

28

30

35

40

mean age:

(at transition)

1st decile at age:

1st quartile at:

median at age:

3rd quartile at:
West

Austria Germany Flanders Italy Spain

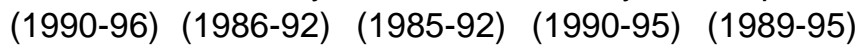

$\begin{array}{ccccc}0 & 0 & 0 & 0 & 0 \\ 0 & 0 & 0 & 0 & 0 \\ 0 & 0 & 0 & 0 & 1 \\ 1 & 1 & 1 & 0 & 2 \\ 3 & 3 & 7 & 2 & 6 \\ 7 & 5 & 12 & 4 & 9 \\ 10 & 6 & 18 & 7 & 13 \\ 17 & 12 & 37 & 14 & 22 \\ 26 & 21 & 51 & 25 & 37 \\ 37 & 35 & 68 & 50 & 61 \\ 43 & 38 & 71 & 58 & 68 \\ & & & & \\ 29 & 30 & 28 & 31 & 30 \\ & & & & \\ & & & & \\ 26 & 28 & 25 & 28 & 26 \\ 30 & 31 & 27 & 30 & 29 \\ -- & -- & 30 & 35 & 33 \\ -- & -- & -- & -- & --\end{array}$


Table 15: Cumulative percent ever "having a first birth ever in any marriage" / Men

$\begin{array}{lccccc}\text { age } & \begin{array}{c}\text { GDR } \\ (1984-89)\end{array} & \begin{array}{c}\text { Hungary } \\ (1988-93)\end{array} & \begin{array}{c}\text { Czech R } \\ (\mathrm{NA})\end{array} & \begin{array}{c}\text { Slovenia } \\ (1989-95)\end{array} & \begin{array}{c}\text { Latvia } \\ (1989-95)\end{array} \\ 16 & 0 & 0 & & & \\ 18 & 0 & 0 & & 0 & 0 \\ 20 & 0 & 2 & 0 & 5 \\ 22 & 5 & 6 & 2 & 14 \\ 24 & 15 & 21 & 16 & 33 \\ 25 & 20 & 29 & 20 & 43 \\ 26 & 25 & 36 & 27 & 50 \\ 28 & 36 & 48 & 39 & 62 \\ 30 & 42 & 57 & 50 & 67 \\ 35 & 51 & 68 & 61 & 70 \\ 40 & -- & 72 & 66 & 71 \\ & & & & \\ \text { mean age: } & 27 & 27 & & & \\ \text { (at transition) } & & & & & \\ & & & & & \\ \text { 1st decile at age: } & 24 & 23 & & 24 & 21 \\ \text { 1st quartile at: } & 26 & 25 & -- & -\end{array}$

$\begin{array}{ccc}\text { Lithuania } & \text { Poland } \\ (1989-95) & \text { (NA) }\end{array}$

$\begin{array}{lc}16 & 0 \\ 18 & 0 \\ 20 & 4 \\ 22 & 14 \\ 24 & 34 \\ 25 & 43 \\ 26 & 51 \\ 28 & 65 \\ 30 & 74 \\ 35 & 81 \\ 40 & 82 \\ & \\ \text { mean age: } & 25 \\ \text { (at transition) } & \\ & \\ \text { 1st decile at age: } & 22 \\ \text { 1st quartile at: } & 24 \\ \text { median at age: } & 26 \\ \text { 3rd quartile at: } & 31\end{array}$


Table 15: Cumulative percent ever "having a first birth ever in any marriage" / Women

\begin{tabular}{|c|c|c|c|c|c|}
\hline age & $\begin{array}{l}\text { Sweden } \\
(1978-93)\end{array}$ & $\begin{array}{c}\text { Norway } \\
(1974-89)\end{array}$ & $\begin{array}{l}\text { Finland } \\
(1979-89)\end{array}$ & $\begin{array}{c}\text { France } \\
(1988-94)\end{array}$ & $\begin{array}{c}\text { USA } \\
(1989-95)\end{array}$ \\
\hline 16 & 0 & 0 & 0 & 0 & 0 \\
\hline 18 & 0 & 1 & 1 & 0 & 2 \\
\hline 20 & 1 & 5 & 4 & 2 & 6 \\
\hline 22 & 2 & 14 & 11 & 3 & 12 \\
\hline 24 & 5 & 24 & 19 & 12 & 19 \\
\hline 25 & 7 & 29 & 25 & 17 & 22 \\
\hline 26 & 9 & 35 & 30 & 22 & 26 \\
\hline 28 & 15 & 45 & 42 & 31 & 34 \\
\hline 30 & 20 & 52 & 51 & 39 & 41 \\
\hline 35 & 26 & 60 & 60 & 47 & 53 \\
\hline 40 & 28 & 63 & 64 & 51 & 57 \\
\hline $\begin{array}{l}\text { mean age: } \\
\text { (at transition) }\end{array}$ & 28 & 26 & 26 & 27 & 27 \\
\hline 1st decile at age: & 27 & 22 & 22 & 24 & 22 \\
\hline 1st quartile at: & 33 & 25 & 25 & 27 & 26 \\
\hline median at age: & -- & 30 & 30 & 38 & 34 \\
\hline 3rd quartile at: & -- & -- & -- & -- & -- \\
\hline age & $\begin{array}{c}\text { Austria } \\
(1990-96)\end{array}$ & $\begin{array}{c}\text { West } \\
\text { Germany } \\
(1986-92)\end{array}$ & $\begin{array}{c}\text { Flanders } \\
(1985-92)\end{array}$ & $\begin{array}{c}\text { Italy } \\
(1990-95)\end{array}$ & $\begin{array}{c}\text { Spain } \\
(1989-95)\end{array}$ \\
\hline
\end{tabular}

$\begin{array}{lccccc}16 & 0 & 0 & 0 & 1 & 0 \\ 18 & 1 & 0 & 0 & 1 & 1 \\ 20 & 3 & 2 & 1 & 3 & 4 \\ 22 & 8 & 6 & 6 & 6 & 11 \\ 24 & 18 & 12 & 16 & 12 & 19 \\ 25 & 23 & 15 & 25 & 17 & 22 \\ 26 & 29 & 21 & 34 & 23 & 27 \\ 28 & 39 & 29 & 53 & 34 & 40 \\ 30 & 45 & 37 & 64 & 47 & 55 \\ 35 & 52 & 46 & 73 & 66 & 73 \\ 40 & 54 & 48 & 74 & 72 & 78 \\ & & & & & \\ \text { mean age: } & 26 & 27 & 27 & 28 & 28 \\ \text { (at transition) } & & & & & \\ \text { 1st decile at age: } & & & & & \\ \text { 1st quartile at: } & 23 & 24 & 23 & 24 & 22 \\ \text { median at age: } & 26 & 27 & 25 & 27 & 26 \\ \text { 3rd quartile at: } & 33 & -- & 28 & 31 & 30 \\ & -- & -- & -- & -- & 36\end{array}$


Table 15: Cumulative percent ever "having a first birth ever in any marriage" / Women

\begin{tabular}{|c|c|c|c|c|c|}
\hline age & $\begin{array}{c}\text { GDR } \\
(1984-89)\end{array}$ & $\begin{array}{c}\text { Hungary } \\
(1988-93)\end{array}$ & $\begin{array}{c}\text { Czech R } \\
(1992-97)\end{array}$ & $\begin{array}{c}\text { Slovenia } \\
(1989-95)\end{array}$ & $\begin{array}{c}\text { Latvia } \\
(1989-95)\end{array}$ \\
\hline 16 & 0 & 0 & 0 & 0 & 0 \\
\hline 18 & 0 & 2 & 1 & 1 & 2 \\
\hline 20 & 4 & 13 & 14 & 5 & 15 \\
\hline 22 & 17 & 32 & 31 & 18 & 33 \\
\hline 24 & 30 & 49 & 43 & 35 & 47 \\
\hline 25 & 35 & 57 & 51 & 41 & 51 \\
\hline 26 & 39 & 64 & 57 & 46 & 54 \\
\hline 28 & 43 & 72 & 66 & 53 & 61 \\
\hline 30 & 45 & 76 & 71 & 57 & 64 \\
\hline 35 & 47 & 81 & 75 & 60 & 68 \\
\hline 40 & -- & 83 & 76 & 62 & 69 \\
\hline $\begin{array}{l}\text { mean age: } \\
\text { (at transition) }\end{array}$ & 23 & 24 & 24 & 24 & 23 \\
\hline 1st decile at age: & 21 & 20 & 20 & 21 & 20 \\
\hline 1st quartile at: & 23 & 22 & 22 & 23 & 21 \\
\hline median at age: & -- & 25 & 25 & 27 & 25 \\
\hline 3rd quartile at: & -- & 30 & 35 & -- & -- \\
\hline age & $\begin{array}{l}\text { Lithuania } \\
(1989-95)\end{array}$ & $\begin{array}{l}\text { Poland } \\
(1986-91)\end{array}$ & & & \\
\hline 16 & 0 & 0 & & & \\
\hline 18 & 2 & 1 & & & \\
\hline 20 & 12 & 11 & & & \\
\hline 22 & 37 & 31 & & & \\
\hline 24 & 58 & 50 & & & \\
\hline 25 & 65 & 56 & & & \\
\hline 26 & 70 & 62 & & & \\
\hline 28 & 76 & 71 & & & \\
\hline 30 & 79 & 74 & & & \\
\hline 35 & 83 & 78 & & & \\
\hline 40 & 84 & 79 & & & \\
\hline $\begin{array}{l}\text { mean age: } \\
\text { (at transition) }\end{array}$ & 23 & 24 & & & \\
\hline 1st decile at age: & 20 & 20 & & & \\
\hline 1st quartile at: & 22 & 22 & & & \\
\hline median at age: & 24 & 24 & & & \\
\hline 3rd quartile at: & 28 & 31 & & & \\
\hline
\end{tabular}


Table 16: Cumulative percent ever "having a first birth ever out of a union" / Men

$\begin{array}{lcccc}\text { age } & \begin{array}{c}\text { Sweden } \\ (1978-93)\end{array} & \begin{array}{c}\text { Norway } \\ (1974-89)\end{array} & \begin{array}{c}\text { Finland } \\ (1983-92)\end{array} & \begin{array}{c}\text { France } \\ (1988-94)\end{array} \\ 16 & 0 & 0 & 0 & 0 \\ 18 & 0 & 0 & 0 & 0 \\ 20 & 0 & 1 & 0 & 1 \\ 22 & 1 & 2 & 1 & 1 \\ 24 & 1 & 3 & 1 & 1 \\ 25 & 1 & 3 & 1 & 1 \\ 26 & 2 & 3 & 1 & 1 \\ 28 & 2 & 4 & 1 & 2 \\ 30 & 2 & 4 & 2 & 2 \\ 35 & 3 & 5 & 2 & 2 \\ 40 & 3 & 6 & 2 & 2 \\ & & & & \\ \text { mean age: } & 26 & 25 & 27 & 23 \\ \text { (at transition) } & & & & \\ \text { 1st decile at age: } & -- & -- & -- & - \\ \text { 1st quartile at: } & -- & -- & -- & -- \\ \text { median at age: } & -- & -- & -- & - \\ \text { 3rd quartile at: } & -- & -- & -- & --\end{array}$

age

16

18

20

22

24

25

26

28

30

35

40

mean age:

(at transition)

1st decile at age:

1st quartile at:

median at age:

3rd quartile at:
West

Austria Germany Flanders Italy Spain

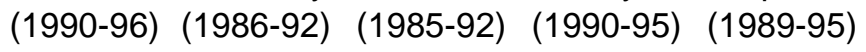

$\begin{array}{lllll}0 & 0 & 0 & 0 & 0 \\ 0 & 0 & 0 & 0 & 1 \\ 1 & 0 & 1 & 0 & 1 \\ 3 & 1 & 1 & 0 & 1 \\ 4 & 1 & 1 & 0 & 1 \\ 5 & 1 & 1 & 0 & 2 \\ 5 & 2 & 1 & 0 & 2 \\ 6 & 2 & 1 & 0 & 2 \\ 7 & 2 & 1 & 0 & 2 \\ 7 & 2 & 1 & 1 & 2 \\ 7 & 3 & 1 & 1 & 2 \\ & & & & \\ 24 & 26 & 22 & 29 & 21 \\ & & & & \\ -- & -- & -- & -- & -- \\ -- & -- & -- & -- & -- \\ -- & -- & -- & -- & - \\ -- & -- & -- & -- & -\end{array}$


Table 16: Cumulative percent ever "having a first birth ever out of a union" / Men

\begin{tabular}{|c|c|c|c|c|c|}
\hline age & $\begin{array}{c}\text { GDR } \\
(1984-89)\end{array}$ & $\begin{array}{c}\text { Hungary } \\
(1988-93)\end{array}$ & $\begin{array}{c}\text { Czech R } \\
\text { (NA) }\end{array}$ & $\begin{array}{c}\text { Slovenia } \\
(1989-95)\end{array}$ & $\begin{array}{c}\text { Latvia } \\
(1989-95)\end{array}$ \\
\hline 16 & 0 & 0 & & 0 & 0 \\
\hline 18 & 1 & 0 & & 0 & 0 \\
\hline 20 & 2 & 0 & & 0 & 1 \\
\hline 22 & 7 & 1 & & 1 & 1 \\
\hline 24 & 10 & 2 & & 2 & 3 \\
\hline 25 & 12 & 2 & & 3 & 3 \\
\hline 26 & 14 & 2 & & 4 & 3 \\
\hline 28 & 15 & 2 & & 5 & 3 \\
\hline 30 & 16 & 2 & & 5 & 3 \\
\hline 35 & 17 & 2 & & 5 & 3 \\
\hline 40 & -- & 2 & & 5 & 3 \\
\hline $\begin{array}{l}\text { mean age: } \\
\text { (at transition) }\end{array}$ & 23 & 22 & & 24 & 23 \\
\hline 1st decile at age: & 24 & -- & & -- & -- \\
\hline 1st quartile at: & -- & -- & & -- & -- \\
\hline median at age: & -- & -- & & -- & -- \\
\hline 3rd quartile at: & -- & -- & & -- & -- \\
\hline
\end{tabular}

$\begin{array}{ccc}\text { Lithuania } & \text { Poland } \\ (1989-95) & \text { (NA) }\end{array}$

$\begin{array}{ll}16 & 0 \\ 18 & 0 \\ 20 & 1 \\ 22 & 1 \\ 24 & 2 \\ 25 & 3 \\ 26 & 3 \\ 28 & 3 \\ 30 & 3 \\ 35 & 3 \\ 40 & 3 \\ & \\ \text { mean age: } & \\ \text { (at transition) } & 22 \\ & \\ \text { 1st decile at age: } & -- \\ \text { 1st quartile at: } & - \\ \text { median at age: } & - \\ \text { 3rd quartile at: } & --\end{array}$


Table 16: Cumulative percent ever "having a first birth ever out of a union" / Women

\begin{tabular}{|c|c|c|c|c|c|}
\hline age & $\begin{array}{l}\text { Sweden } \\
(1978-93)\end{array}$ & $\begin{array}{c}\text { Norway } \\
(1974-89)\end{array}$ & $\begin{array}{c}\text { Finland } \\
(1979-89)\end{array}$ & $\begin{array}{c}\text { France } \\
(1988-94)\end{array}$ & $\begin{array}{c}\text { USA } \\
(1989-95)\end{array}$ \\
\hline 16 & 0 & 0 & 0 & 0 & 1 \\
\hline 18 & 0 & 1 & 0 & 1 & 5 \\
\hline 20 & 1 & 4 & 2 & 2 & 11 \\
\hline 22 & 2 & 5 & 2 & 3 & 14 \\
\hline 24 & 3 & 6 & 3 & 4 & 17 \\
\hline 25 & 3 & 6 & 3 & 4 & 17 \\
\hline 26 & 3 & 7 & 3 & 4 & 18 \\
\hline 28 & 4 & 7 & 3 & 5 & 18 \\
\hline 30 & 4 & 7 & 4 & 5 & 19 \\
\hline 35 & 4 & 8 & 4 & 6 & 19 \\
\hline 40 & 5 & 8 & 4 & 6 & 20 \\
\hline $\begin{array}{l}\text { mean age: } \\
\text { (at transition) }\end{array}$ & 25 & 22 & 24 & 23 & 21 \\
\hline 1st decile at age: & -- & -- & -- & -- & 20 \\
\hline 1st quartile at: & -- & -- & -- & -- & -- \\
\hline median at age: & -- & -- & -- & -- & -- \\
\hline 3rd quartile at: & -- & -- & -- & -- & -- \\
\hline age & $\begin{array}{c}\text { Austria } \\
(1990-96)\end{array}$ & $\begin{array}{c}\text { West } \\
\text { Germany } \\
(1986-92)\end{array}$ & $\begin{array}{c}\text { Flanders } \\
\text { (1985-92) }\end{array}$ & $\begin{array}{c}\text { Italy } \\
(1990-95)\end{array}$ & $\begin{array}{c}\text { Spain } \\
(1989-95)\end{array}$ \\
\hline
\end{tabular}

$\begin{array}{lccccc}16 & 0 & 0 & 0 & 0 & 0 \\ 18 & 1 & 0 & 0 & 1 & 1 \\ 20 & 5 & 1 & 0 & 1 & 1 \\ 22 & 8 & 2 & 0 & 1 & 2 \\ 24 & 9 & 2 & 1 & 1 & 2 \\ 25 & 11 & 3 & 1 & 1 & 2 \\ 26 & 11 & 3 & 1 & 1 & 2 \\ 28 & 13 & 4 & 1 & 1 & 3 \\ 30 & 13 & 4 & 1 & 1 & 3 \\ 35 & 14 & 5 & 1 & 3 & 3 \\ 40 & 14 & 5 & 1 & 3 & 3 \\ & & & & & \\ \text { mean age: } & & & & \\ \text { (at transition) } & 22 & 25 & 23 & 26 & 23 \\ \text { 1st decile at age: } & & & & & - \\ \text { 1st quartile at: } & 25 & -- & -- & -- & - \\ \text { median at age: } & -- & -- & -- & -- & - \\ \text { 3rd quartile at: } & -- & -- & -- & -- & - \\ & -- & -- & -- & -- & -\end{array}$


Table 16: Cumulative percent ever "having a first birth ever out of a union" / Women

$\begin{array}{lccccc}\text { age } & \begin{array}{c}\text { GDR } \\ (1984-89)\end{array} & \begin{array}{c}\text { Hungary } \\ (1988-93)\end{array} & \begin{array}{c}\text { Czech R } \\ (1992-97)\end{array} & \begin{array}{c}\text { Slovenia } \\ (1989-95)\end{array} & \begin{array}{c}\text { Latvia } \\ (1989-95)\end{array} \\ 16 & & & & & \\ 18 & 0 & 0 & 0 & 0 & 0 \\ 20 & 2 & 1 & 1 & 1 & 2 \\ 22 & 8 & 2 & 3 & 3 & 5 \\ 24 & 16 & 3 & 4 & 6 & 7 \\ 25 & 20 & 4 & 5 & 8 & 8 \\ 26 & 21 & 4 & 6 & 8 & 9 \\ 28 & 22 & 4 & 6 & 8 & 10 \\ 30 & 23 & 4 & 7 & 9 & 11 \\ 35 & 24 & 4 & 7 & 9 & 11 \\ 40 & 25 & 5 & 7 & 10 & 12 \\ & -- & 5 & 7 & 10 & 12 \\ \text { mean age: } & & & & & \\ \text { (at transition) } & 22 & 22 & 21 & 23 & 22 \\ & & & & & \\ \text { 1st decile at age: } & 21 & -- & -- & 32 & 26 \\ \text { 1st quartile at: } & 33 & -- & -- & -- & -- \\ \text { median at age: } & -- & -- & -- & -- & - \\ \text { 3rd quartile at: } & -- & -- & -- & -- & --\end{array}$

$\begin{array}{ccc}\text { Lithuania } & \text { Poland } \\ & (1989-95) & (1986-91)\end{array}$

$\begin{array}{lcc}16 & 0 & 0 \\ 18 & 0 & 1 \\ 20 & 2 & 3 \\ 22 & 3 & 6 \\ 24 & 4 & 8 \\ 25 & 5 & 8 \\ 26 & 5 & 9 \\ 28 & 6 & 10 \\ 30 & 6 & 10 \\ 35 & 6 & 11 \\ 40 & 6 & 11 \\ & & \\ \text { mean age: } & 23 & 23 \\ \text { (at transition) } & & \\ & & \\ \text { 1st decile at age: } & -- & 28 \\ \text { 1st quartile at: } & -- & -- \\ \text { median at age: } & -- & -- \\ \text { 3rd quartile at: } & -- & -\end{array}$


Table 17: Cumulative percent parents, by time since formation of a union by a childless couple, single-decrement life-table method with censoring at union dissolution

duration in years

Sweden Norway Finland France USA

$(1985-93) \quad(1983-89) \quad(1983-92) \quad(1988-94) \quad(1989-95)$

$\begin{array}{lccccc}0 & 0 & 0 & 0 & 0 & 0 \\ 1 & 8 & 14 & 11 & 8 & 14 \\ 2 & 23 & 31 & 26 & 24 & 30 \\ 3 & 38 & 46 & 36 & 39 & 41 \\ 4 & 51 & 57 & 48 & 51 & 52 \\ 5 & 61 & 66 & 56 & 62 & 62 \\ 7 & 75 & 79 & 69 & 75 & 74 \\ 10 & 86 & 86 & 79 & 86 & 82 \\ 15 & 91 & 88 & 85 & 93 & 86\end{array}$

$\begin{array}{llllll}\text { mean dur: } & 4 & 4 & 4 & 4 & 4\end{array}$ (at first childbirth / conditional on childbirth and disregarding union disruption)

1st decile at dur:

1st quartile at:

median at dur:

3rd quartile at:

$\begin{array}{lllll}2 & 1 & 1 & 2 & 1 \\ 3 & 2 & 2 & 3 & 2 \\ 4 & 4 & 5 & 4 & 4 \\ 7 & 7 & 9 & 7 & 8\end{array}$

$\begin{array}{lccccc}\text { duration in years } & \text { Austria } & \text { Germany } & \text { Flanders } & \text { Italy } & \text { Spain } \\ & (1990-96) & (1986-92) & (1985-92) & (1990-95) & (1989-95)\end{array}$

$\begin{array}{lccccc}0 & 0 & 0 & 0 & 0 & 0 \\ 1 & 16 & 14 & 7 & 19 & 21 \\ 2 & 32 & 26 & 25 & 44 & 43 \\ 3 & 47 & 36 & 44 & 61 & 59 \\ 4 & 57 & 46 & 58 & 71 & 70 \\ 5 & 66 & 54 & 68 & 79 & 79 \\ 7 & 79 & 66 & 81 & 87 & 86 \\ 10 & 86 & 75 & 89 & 91 & 91 \\ 15 & 90 & 81 & 91 & 94 & 94\end{array}$

$\begin{array}{llllll}\text { mean dur: } & 4 & 4 & 4 & 3 & 3\end{array}$

(at first childbirth / conditional on childbirth and disregarding union disruption)

1st decile at dur:

1st quartile at:

median at dur:

3rd quartile at:

$\begin{array}{ccccc}1 & 1 & 2 & 1 & 1 \\ 2 & 2 & 2 & 2 & 2 \\ 4 & 5 & 4 & 3 & 3 \\ 7 & 10 & 6 & 5 & 5\end{array}$


Table 17: Cumulative percent parents, by time since formation of a union by a childless couple, single-decrement life-table method with censoring at union dissolution

duration in years

$\begin{array}{ccccc}\text { GDR } & \text { Hungary } & \text { Czech R } & \text { Slovenia } & \text { Latvia } \\ (1984-89) & (1988-93) & (1992-97) & (1989-95) & (1989-95)\end{array}$

$\begin{array}{lccccc}0 & 0 & 0 & 0 & 0 & 0 \\ 1 & 31 & 28 & 34 & 46 & 33 \\ 2 & 57 & 56 & 54 & 69 & 61 \\ 3 & 71 & 72 & 67 & 80 & 75 \\ 4 & 79 & 81 & 77 & 85 & 81 \\ 5 & 84 & 86 & 83 & 88 & 86 \\ 7 & 90 & 90 & 89 & 92 & 88 \\ 10 & 93 & 93 & -- & 94 & 89 \\ 15 & 94 & 95 & -- & 96 & 89\end{array}$

$\begin{array}{llllll}\text { mean dur: } & 2 & 2 & -- & 2 & 2\end{array}$ (at first childbirth / conditional on childbirth and disregarding union disruption)

1st decile at dur:

1st quartile at:

median at dur:

3rd quartile at:

$\begin{array}{lllll}1 & 1 & 1 & 1 & 1 \\ 1 & 1 & 1 & 1 & 1 \\ 2 & 2 & 2 & 2 & 2 \\ 4 & 4 & 4 & 3 & 3\end{array}$

$\begin{array}{lcc}\text { duration in years } & \text { Lithuania } & \text { Poland } \\ & (1989-95) & (1986-91)\end{array}$

$\begin{array}{lcc}0 & 0 & 0 \\ 1 & 37 & 49 \\ 2 & 68 & 81 \\ 3 & 81 & 88 \\ 4 & 85 & 90 \\ 5 & 88 & 93 \\ 7 & 91 & 94 \\ 10 & 92 & 95 \\ 15 & 94 & 96\end{array}$

mean dur:

2

0

49

81

90

93

94

95

(at first childbirth / conditional on childbirth and disregarding union disruption)

1st decile at dur:

1st quartile at:

median at dur:

3rd quartile at:

$\begin{array}{ll}1 & 1 \\ 1 & 1 \\ 2 & 2 \\ 3 & 2\end{array}$


Table 18: Cumulative percent separated, by time since formation of a childless union, single-decrement life-table method with censoring at childbirth

$\begin{array}{lccccc}\text { duration in years } & \text { Sweden } & \text { Norway } & \text { Finland } & \text { France } & \text { USA } \\ & (1985-93) & (1983-89) & (1983-92) & (1988-94) & (1989-95)\end{array}$

$\begin{array}{lccccc}0 & 0 & 0 & 0 & 0 & 0 \\ 1 & 9 & 7 & 6 & 7 & 18 \\ 2 & 19 & 15 & 12 & 15 & 28 \\ 3 & 29 & 23 & 19 & 20 & 36 \\ 4 & 36 & 30 & 25 & 28 & 39 \\ 5 & 42 & 33 & 30 & 34 & 44 \\ 7 & 52 & 40 & 37 & 42 & 49 \\ 10 & 60 & 46 & 43 & 53 & 55 \\ 15 & 72 & 50 & 49 & 62 & 62\end{array}$

mean dur: $\quad \begin{array}{llllll}5 & 5 & 5 & 5 & 4\end{array}$ (at union disruption / conditional on disruption and disregarding childbearing)

1st decile at dur:

1st quartile at:

median at dur:

3rd quartile at:

$\begin{array}{ccccc}2 & 2 & 2 & 2 & 1 \\ 3 & 4 & 4 & 4 & 2 \\ 7 & 15 & -- & 9 & 8 \\ -- & -- & -- & -- & --\end{array}$

duration in years

West

Austria Germany Flanders Italy Spain $(1990-96) \quad(1986-92) \quad(1985-92) \quad(1990-95) \quad(1989-95)$

$\begin{array}{cccccc}0 & 0 & 0 & 0 & 0 & 0 \\ 1 & 4 & 5 & 2 & 4 & 7 \\ 2 & 14 & 13 & 5 & 6 & 10 \\ 3 & 22 & 22 & 7 & 9 & 12 \\ 4 & 27 & 28 & 9 & 10 & 15 \\ 5 & 31 & 33 & 13 & 13 & 19 \\ 7 & 42 & 42 & 19 & 16 & 25 \\ 10 & 49 & 48 & 25 & 19 & 31 \\ 15 & 57 & 54 & 35 & 27 & 33\end{array}$

$\begin{array}{llllll}\text { mean dur: } & 5 & 5 & 7 & 6 & 5\end{array}$ (at union disruption / conditional on disruption and disregarding childbearing)

1st decile at dur:

1st quartile at:

$\begin{array}{ccccc}2 & 2 & 5 & 4 & 2 \\ 4 & 4 & 10 & 15 & 7 \\ 11 & 11 & -- & -- & -- \\ -- & -- & -- & -- & --\end{array}$

3rd quartile at: 
Table 18: Cumulative percent separated, by time since formation of a childless union, single-decrement life-table method with censoring at childbirth

$\begin{array}{lccccc}\text { duration in years } & \text { GDR } & \text { Hungary } & \text { Czech R } & \text { Slovenia } & \text { Latvia } \\ & (1984-89) & (1988-93) & (1992-97) & (1989-95) & (1989-95)\end{array}$

\begin{tabular}{|c|c|c|c|c|c|}
\hline 0 & 0 & 0 & 0 & 0 & 0 \\
\hline 1 & 5 & 5 & 4 & 3 & 10 \\
\hline 2 & 12 & 13 & 10 & 7 & 22 \\
\hline 3 & 20 & 20 & 16 & 15 & 30 \\
\hline 4 & 25 & 26 & 23 & 18 & 43 \\
\hline 5 & 29 & 28 & 26 & 21 & 54 \\
\hline 7 & 37 & 36 & 26 & 24 & 58 \\
\hline 10 & 39 & 48 & -- & 26 & 63 \\
\hline 15 & 47 & 54 & -- & 30 & 68 \\
\hline mean dur: & 5 & 5 & -- & 5 & 4 \\
\hline (at union di & & tion & ireg & childl & \\
\hline 1st decile at dur: & 2 & 2 & 2 & 3 & 1 \\
\hline 1st quartile at: & 4 & 4 & 5 & 8 & 3 \\
\hline median at dur: & -- & 12 & -- & -- & 5 \\
\hline 3rd quartile at: & -- & -- & -- & -- & - \\
\hline
\end{tabular}

$\begin{array}{lcc}\text { duration in years } & \begin{array}{c}\text { Lithuania } \\ (1989-95)\end{array} & \begin{array}{c}\text { Poland } \\ (1986-91)\end{array} \\ 0 & 0 & 0 \\ 1 & 3 & 1 \\ 2 & 6 & 3 \\ 3 & 11 & 3 \\ 4 & 17 & 6 \\ 5 & 27 & 8 \\ 7 & 32 & 8 \\ 10 & 36 & 8 \\ 15 & 43 & 19\end{array}$

mean dur: $\quad 5 \quad 9$

(at union disruption / conditional on disruption and disregarding childbearing)

1st decile at dur: $\quad 3 \quad 11$

1st quartile at: $\quad 5 \quad--$

median at dur: $\quad--\quad \quad--$

3rd quartile at: $\quad$-- $\quad$-- 
Table 19: Cumulative percent married, by time since formation of a consensual union, single-decrement life-table method with censoring at union dissolution

$\begin{array}{lccccc}\text { duration in years } & \text { Sweden } & \text { Norway } & \text { Finland } & \text { France } & \text { USA } \\ & (1985-93) & (1983-89) & (1983-92) & (1988-94) & (1989-95)\end{array}$

$\begin{array}{lccccc}0 & 0 & 0 & 0 & 0 & 0 \\ 1 & 6 & 10 & 15 & 10 & 27 \\ 2 & 14 & 23 & 29 & 25 & 47 \\ 3 & 23 & 38 & 41 & 35 & 59 \\ 4 & 32 & 46 & 49 & 41 & 66 \\ 5 & 40 & 51 & 57 & 45 & 71 \\ 7 & 53 & 63 & 65 & 54 & 80 \\ 10 & 67 & 70 & 72 & 57 & 84 \\ 15 & 78 & -- & 76 & 70 & 91\end{array}$

$\begin{array}{llllll}\text { mean dur: } & 6 & -- & 4 & 5 & 3\end{array}$

(at marriage / conditional on marriage and disregarding union disruption)

1st decile at dur:

1st quartile at:

median at dur:

3rd quartile at:

$\begin{array}{ccccc}2 & 1 & 1 & 1 & 1 \\ 4 & 3 & 2 & 2 & 1 \\ 7 & 5 & 5 & 7 & 3 \\ 13 & 13 & 13 & -- & 6\end{array}$

$\begin{array}{cccccc}\text { duration in years } & \text { Wustria } & \text { Germany } & \text { Flanders } & \text { Italy } & \text { Spain } \\ & (1990-96) & (1986-92) & (1985-92) & (1990-95) & (1989-95)\end{array}$

$\begin{array}{lccccc}0 & 0 & 0 & 0 & 0 & 0 \\ 1 & 9 & 14 & 16 & 16 & 10 \\ 2 & 23 & 30 & 32 & 30 & 22 \\ 3 & 36 & 41 & 44 & 39 & 32 \\ 4 & 44 & 49 & 52 & 47 & 38 \\ 5 & 51 & 56 & 59 & 57 & 44 \\ 7 & 63 & 63 & 68 & 70 & 51 \\ 10 & 75 & 69 & 77 & 79 & 61 \\ 15 & 81 & -- & -- & -- & --\end{array}$

$\begin{array}{lccccc}\begin{array}{l}\text { mean dur: } \\ \text { (at marriage / conditional on marriage and disregarding union disruption) }\end{array} & - \\ & & & & & - \\ \text { 1st decile at dur: } & 2 & 1 & 1 & 1 & 1 \\ \text { 1st quartile at: } & 3 & 2 & 2 & 2 & 3 \\ \text { median at dur: } & 5 & 5 & 4 & 5 & 7 \\ \text { 3rd quartile at: } & 10 & 13 & 9 & 8 & --\end{array}$


Table 19: Cumulative percent married, by time since formation of a consensual union, single-decrement life-table method with censoring at union dissolution

$\begin{array}{cccccc}\text { duration in years } & \text { GDR } & \text { Hungary } & \text { Czech R } & \text { Slovenia } & \text { Latvia } \\ & (1984-89) & (1988-93) & (1992-97) & (1989-95) & (1989-95)\end{array}$

$\begin{array}{lccccc}0 & 0 & 0 & 0 & 0 & 0 \\ 1 & 25 & 25 & 24 & 21 & 30 \\ 2 & 45 & 40 & 51 & 37 & 44 \\ 3 & 56 & 49 & 60 & 47 & 52 \\ 4 & 63 & 53 & 65 & 52 & 57 \\ 5 & 69 & 58 & 67 & 56 & 59 \\ 7 & 74 & 63 & 75 & 65 & 59 \\ 10 & 80 & 67 & -- & 72 & 63 \\ 15 & -- & -- & -- & 73 & --\end{array}$

mean dur: $330 \begin{array}{llll}3 & -- & 3\end{array}$

(at marriage / conditional on marriage and disregarding union disruption)

1st decile at dur:

1st quartile at:

median at dur:

3rd quartile at:

$\begin{array}{lllll}1 & 1 & 1 & 1 & 1 \\ 1 & 1 & 2 & 2 & 1 \\ 3 & 4 & 2 & 4 & 3 \\ 8 & -- & 7 & -- & --\end{array}$

duration in years

Lithuania Poland

(1989-95) (1986-91)

$\begin{array}{lcc}0 & 0 & 0 \\ 1 & 48 & 24 \\ 2 & 59 & 37 \\ 3 & 65 & 43 \\ 4 & 70 & 45 \\ 5 & 73 & 47 \\ 7 & 77 & -- \\ 10 & 77 & - \\ 15 & -- & --\end{array}$

mean dur:

(at marriage / conditional on marriage and disregarding union disruption)

1st decile at dur:

1st quartile at:

median at dur:

0

4

3

45

47

3rd quartile at:

$\begin{array}{ll}1 & 1 \\ 1 & 2 \\ 2 & 6 \\ 6 & --\end{array}$


Table 20: Cumulative percent separated, by time since formation of a consensual union, single-decrement life-table method with censoring at marriage formation

$\begin{array}{lccccc}\text { duration in years } & \text { Sweden } & \text { Norway } & \text { Finland } & \text { France } & \text { USA } \\ & (1985-93) & (1983-89) & (1983-92) & (1988-94) & (1989-95)\end{array}$

$\begin{array}{lccccc}0 & 0 & 0 & 0 & 0 & 0 \\ 1 & 8 & 8 & 6 & 8 & 28 \\ 2 & 19 & 17 & 14 & 16 & 46 \\ 3 & 28 & 27 & 22 & 23 & 57 \\ 4 & 36 & 34 & 32 & 31 & 64 \\ 5 & 41 & 38 & 37 & 37 & 69 \\ 7 & 50 & 47 & 46 & 48 & 76 \\ 10 & 56 & 55 & 53 & 56 & 85 \\ 15 & 64 & -- & 60 & 66 & 91\end{array}$

mean dur: $\quad 5 \quad--\quad 5 \quad 5 \quad 5 \quad 3$ (at union disruption / conditional on disruption and disregarding marriage formation)

1st decile at dur:

1st quartile at:

median at dur:

3rd quartile at:

$\begin{array}{lllll}2 & 2 & 2 & 2 & 1 \\ 3 & 3 & 4 & 4 & 1 \\ 7 & 9 & 9 & 8 & 3 \\ -- & -- & -- & -- & 7\end{array}$

\begin{tabular}{cccccc} 
& \multicolumn{5}{c}{ West } \\
duration in years & Austria & Germany & Flanders & Italy & Spain \\
& $(1990-96)$ & $(1986-92)$ & $(1985-92)$ & $(1990-95)$ & $(1989-95)$
\end{tabular}

$\begin{array}{lccccc}0 & 0 & 0 & 0 & 0 & 0 \\ 1 & 4 & 6 & 4 & 19 & 22 \\ 2 & 14 & 17 & 10 & 26 & 32 \\ 3 & 23 & 30 & 16 & 35 & 37 \\ 4 & 29 & 37 & 20 & 39 & 43 \\ 5 & 33 & 44 & 27 & 42 & 49 \\ 7 & 44 & 54 & 36 & 46 & 57 \\ 10 & 45 & 61 & 40 & 51 & 64 \\ 15 & 55 & -- & -- & -- & --\end{array}$

mean dur:

64

(at union disruption / conditional on disruption and disregarding marriage formation)

1st decile at dur:

1st quartile at:

median at dur:

3rd quartile at:

$\begin{array}{lllcc}2 & 2 & 2 & 1 & 1 \\ 4 & 3 & 5 & 2 & 2 \\ 8 & 7 & -- & 10 & 6 \\ -- & -- & -- & -- & --\end{array}$


Table 20: Cumulative percent separated, by time since formation of a consensual union, single-decrement life-table method with censoring at marriage formation

$\begin{array}{lccccc}\text { duration in years } & \text { GDR } & \text { Hungary } & \text { Czech R } & \text { Slovenia } & \text { Latvia } \\ & (1984-89) & (1988-93) & (1992-97) & (1989-95) & (1989-95)\end{array}$

$\begin{array}{lccccc}0 & 0 & 0 & 0 & 0 & 0 \\ 1 & 9 & 11 & 8 & 4 & 15 \\ 2 & 19 & 26 & 15 & 8 & 30 \\ 3 & 29 & 35 & 25 & 15 & 37 \\ 4 & 40 & 45 & 30 & 18 & 48 \\ 5 & 42 & 50 & 37 & 22 & 56 \\ 7 & 50 & 56 & 40 & 24 & 67 \\ 10 & 50 & 68 & -- & 28 & 76 \\ 15 & -- & -- & -- & 35 & --\end{array}$

mean dur: $\quad 4 \quad--\quad 5 \quad--\quad 5$

(at union disruption / conditional on disruption and disregarding marriage formation)

1st decile at dur:

1st quartile at:

median at dur:

3rd quartile at:

$\begin{array}{ccccc}2 & 1 & 2 & 3 & 1 \\ 3 & 2 & 3 & 8 & 2 \\ 7 & 5 & -- & -- & 5 \\ -- & -- & -- & -- & 10\end{array}$

$$
\begin{array}{lcc}
\text { duration in years } & \text { Lithuania } & \text { Poland } \\
& (1989-95) & (1986-91)
\end{array}
$$

$\begin{array}{lcc}0 & 0 & 0 \\ 1 & 11 & 2 \\ 2 & 21 & 7 \\ 3 & 33 & 7 \\ 4 & 42 & 7 \\ 5 & 52 & 7 \\ 7 & 64 & -- \\ 10 & 68 & -- \\ 15 & -- & --\end{array}$

mean dur:

(at union disruption / conditional on disruption and disregarding marriage formation)

1st decile at dur:

1st quartile at:

median at dur:

3rd quartile at: 
Table 21: Cumulative percent married, by time since formation of a consensual union, competing-risks life-table method with union dissolution as a competing event

$\begin{array}{lccccc}\text { duration in years } & \text { Sweden } & \text { Norway } & \text { Finland } & \text { France } & \text { USA } \\ & (1985-93) & (1983-89) & (1983-92) & (1988-94) & (1989-95)\end{array}$

$\begin{array}{lccccc}0 & 0 & 0 & 0 & 0 & 0 \\ 1 & 6 & 10 & 15 & 10 & 23 \\ 2 & 13 & 21 & 27 & 22 & 36 \\ 3 & 20 & 32 & 37 & 31 & 42 \\ 4 & 26 & 38 & 43 & 35 & 45 \\ 5 & 31 & 42 & 48 & 38 & 46 \\ 7 & 38 & 48 & 53 & 43 & 49 \\ 10 & 44 & 52 & 56 & 45 & 50 \\ 15 & 49 & -- & 58 & 50 & 50 \\ & & & & & \\ \text { mean dur: } & 5 & -- & 3 & 4 & 2 \\ \text { (at marriage / conditional on marriage) } & & & & \\ & & & & & \\ \text { 1st decile at dur: } & 2 & 1 & 1 & 1 & 1 \\ \text { 1st quartile at: } & 4 & 3 & 2 & 3 & 2 \\ \text { median at dur: } & -- & 8 & 6 & 14 & 10 \\ \text { 3rd quartile at: } & -- & -- & -- & -- & --\end{array}$

$\begin{array}{cccccc} & \text { West } & & \\ \text { duration in years } & \text { Austria } & \text { Germany } & \text { Flanders } & \text { Italy } & \text { Spain } \\ & (1990-96) & (1986-92) & (1985-92) & (1990-95) & (1989-95)\end{array}$

$\begin{array}{lccccc}0 & 0 & 0 & 0 & 0 & 0 \\ 1 & 9 & 14 & 16 & 14 & 9 \\ 2 & 21 & 28 & 30 & 26 & 18 \\ 3 & 33 & 36 & 41 & 32 & 24 \\ 4 & 38 & 42 & 47 & 37 & 28 \\ 5 & 43 & 46 & 53 & 43 & 31 \\ 7 & 50 & 50 & 59 & 50 & 34 \\ 10 & 56 & 52 & 65 & 55 & 38 \\ 15 & 59 & -- & -- & -- & -- \\ & & & & & \\ \text { mean dur: } & & & & & -- \\ \text { (at marriage / conditional on marriage) } & 3 & -- & -- & \\ & & & & & \\ \text { 1st decile at dur: } & 2 & 1 & 1 & 1 & 2 \\ \text { 1st quartile at: } & 3 & 2 & 2 & 2 & 4 \\ \text { median at dur: } & 7 & 7 & 5 & 7 & -- \\ \text { 3rd quartile at: } & -- & -- & -- & -- & -\end{array}$


Table 21: Cumulative percent married, by time since formation of a consensual union, competing-risks life-table method with union dissolution as a competing event
duration in years
GDR Hungary Czech R Slovenia Latvia
(1984-89) (1988-93) (1992-97) (1989-95) (1989-95)

$\begin{array}{lccccc}0 & 0 & 0 & 0 & 0 & 0 \\ 1 & 24 & 24 & 24 & 20 & 28 \\ 2 & 41 & 36 & 47 & 36 & 39 \\ 3 & 49 & 42 & 54 & 45 & 44 \\ 4 & 54 & 44 & 58 & 49 & 47 \\ 5 & 58 & 47 & 59 & 52 & 48 \\ 7 & 60 & 50 & 64 & 59 & 48 \\ 10 & 63 & 51 & -- & 64 & 49 \\ 15 & -- & -- & -- & 65 & -- \\ & & & & & \\ \text { mean dur: } & 2 & -- & -- & 3 & -- \\ \text { (at marriage / conditional on marriage) } & & & & \\ & & & & & \\ \text { 1st decile at dur: } & 1 & 1 & 1 & 1 & 1 \\ \text { 1st quartile at: } & 2 & 2 & 2 & 2 & 1 \\ \text { median at dur: } & 4 & 7 & 3 & 5 & - \\ \text { 3rd quartile at: } & -- & -- & -- & -- & -\end{array}$

$$
\begin{array}{cc}
\text { Lithuania } & \text { Poland } \\
(1989-95) & (1986-91)
\end{array}
$$

$\begin{array}{lcc}0 & 0 & 0 \\ 1 & 45 & 24 \\ 2 & 55 & 36 \\ 3 & 59 & 41 \\ 4 & 62 & 43 \\ 5 & 64 & 46 \\ 7 & 66 & -- \\ 10 & 66 & -- \\ 15 & -- & --\end{array}$

mean dur:

(at marriage / conditional on marriage)

1st decile at dur:

1st quartile at:

median at dur:

3rd quartile at: 
Table 22: Cumulative percent separated, by time since formation of a consensual union, competing-risks life-table method with marriage formation as a competing event

$\begin{array}{lccccc}\text { duration in years } & \text { Sweden } & \text { Norway } & \text { Finland } & \text { France } & \text { USA } \\ & (1985-93) & (1983-89) & (1983-92) & (1988-94) & (1989-95)\end{array}$

$\begin{array}{lccccc}0 & 0 & 0 & 0 & 0 & 0 \\ 1 & 8 & 8 & 6 & 8 & 24 \\ 2 & 17 & 15 & 12 & 15 & 35 \\ 3 & 25 & 22 & 17 & 19 & 41 \\ 4 & 31 & 26 & 22 & 24 & 43 \\ 5 & 34 & 28 & 25 & 28 & 45 \\ 7 & 39 & 32 & 28 & 33 & 46 \\ 10 & 41 & 35 & 30 & 36 & 48 \\ 15 & 43 & -- & 32 & 40 & 49\end{array}$

mean dur:

3

4

(at union disruption / conditional on disruption)

1st decile at dur:

1st quartile at:

median at dur:

3rd quartile at:

$\begin{array}{lllll}2 & 2 & 2 & 2 & 1 \\ 3 & 4 & 5 & 5 & 2 \\ -- & -- & -- & -- & -- \\ -- & -- & -- & -- & --\end{array}$

$\begin{array}{lccccc}\text { duration in years } & \text { Austria } & \text { Germany } & \text { Flanders } & \text { Italy } & \text { Spain } \\ & (1990-96) & (1986-92) & (1985-92) & (1990-95) & (1989-95)\end{array}$

$\begin{array}{lccccc}0 & 0 & 0 & 0 & 0 & 0 \\ 1 & 4 & 6 & 4 & 18 & 21 \\ 2 & 12 & 14 & 8 & 23 & 29 \\ 3 & 18 & 22 & 12 & 29 & 33 \\ 4 & 22 & 27 & 14 & 31 & 37 \\ 5 & 24 & 29 & 17 & 32 & 40 \\ 7 & 29 & 34 & 21 & 34 & 44 \\ 10 & 33 & 36 & 22 & 35 & 48 \\ 15 & 33 & -- & -- & -- & --\end{array}$

mean dur:

4

3

(at union disruption / conditional on disruption)

1st decile at dur:

1st quartile at:

$\begin{array}{lllll}2 & 2 & 3 & 1 & 1 \\ 6 & 4 & -- & 3 & 2 \\ -- & -- & -- & -- & -- \\ -- & -- & -- & -- & --\end{array}$

3rd quartile at: 
Table 22: Cumulative percent separated, by time since formation of a consensual union, competing-risks life-table method with marriage formation as a competing event

duration in years

$$
\begin{array}{ccccc}
\text { GDR } & \text { Hungary } & \text { Czech R } & \text { Slovenia } & \text { Latvia } \\
(1984-89) & (1988-93) & (1992-97) & (1989-95) & (1989-95)
\end{array}
$$

$\begin{array}{cccccc}0 & 0 & 0 & 0 & 0 & 0 \\ 1 & 8 & 10 & 7 & 3 & 12 \\ 2 & 14 & 20 & 11 & 6 & 22 \\ 3 & 19 & 25 & 16 & 10 & 26 \\ 4 & 24 & 30 & 18 & 12 & 31 \\ 5 & 25 & 32 & 20 & 14 & 34 \\ 7 & 27 & 34 & 21 & 15 & 38 \\ 10 & 27 & 38 & -- & 16 & 42 \\ 15 & -- & -- & -- & 18 & --\end{array}$

mean dur:

3

(at union disruption / conditional on disruption)

1st decile at dur:

1st quartile at:

21

median at dur:

3rd quartile at:$$
5
$$

$--\quad--$

duration in years

$$
\begin{array}{cc}
\text { Lithuania } & \text { Poland } \\
(1989-95) & (1986-91)
\end{array}
$$

$\begin{array}{lll}0 & 0 & 0 \\ 1 & 8 & 2 \\ 2 & 13 & 5 \\ 3 & 17 & 5 \\ 4 & 20 & 5 \\ 5 & 23 & 5 \\ 7 & 26 & -- \\ 10 & 27 & -- \\ 15 & -- & --\end{array}$

mean dur:

(at union disruption / conditional on disruption)

1st decile at dur:

1st quartile at:

$\begin{array}{ll}2 & -- \\ 6 & -- \\ -- & -- \\ -- & --\end{array}$

3rd quartile at: 
$=>$ Table 23: Cumulative percent no longer in a consensual union, by time since union formation

$\begin{array}{lccccc}\text { Duration in years } & \text { Sweden } & \text { Norway } & \text { Finland } & \text { France } & \text { USA } \\ & (1985-93) & (1983-89) & (1983-92) & (1988-94) & (1989-95)\end{array}$

$\begin{array}{lccccc}0 & 0 & 0 & 0 & 0 & 0 \\ 1 & 14 & 18 & 21 & 17 & 48 \\ 2 & 30 & 36 & 39 & 37 & 71 \\ 3 & 45 & 54 & 54 & 50 & 82 \\ 4 & 57 & 65 & 65 & 60 & 88 \\ 5 & 65 & 70 & 73 & 65 & 91 \\ 7 & 77 & 80 & 81 & 76 & 95 \\ 10 & 86 & 87 & 87 & 81 & 98 \\ 15 & 92 & -- & 90 & 90 & 99\end{array}$

\begin{tabular}{|c|c|c|c|c|}
\hline $\begin{array}{l}\text { mean dur: } \\
\text { (at exit /conditional on exit) }\end{array}$ & 4 & -- & 3 & 4 \\
\hline 1st decile at dur: & 1 & 1 & 1 & 1 \\
\hline 1st quartile at: & 2 & 2 & 2 & 2 \\
\hline median at dur: & 4 & 3 & 3 & 3 \\
\hline 3rd quartile at: & 7 & 6 & 6 & 7 \\
\hline $\begin{array}{l}\text { mean dur: } \\
\text { (of all episodes / truncated }\end{array}$ & 5 & -- & 4 & 5 \\
\hline
\end{tabular}

$\begin{array}{cccccc} & \text { West } & & \\ \text { duration in years } & \text { Austria } & \text { Germany } & \text { Flanders } & \text { Italy } & \text { Spain } \\ & (1990-96) & (1986-92) & (1985-92) & (1990-95) & (1989-95)\end{array}$

$\begin{array}{lccccc}0 & 0 & 0 & 0 & 0 & 0 \\ 1 & 13 & 19 & 20 & 32 & 30 \\ 2 & 34 & 42 & 39 & 48 & 47 \\ 3 & 51 & 59 & 53 & 61 & 57 \\ 4 & 60 & 68 & 61 & 68 & 65 \\ 5 & 67 & 75 & 70 & 75 & 72 \\ 7 & 79 & 83 & 80 & 84 & 79 \\ 10 & 89 & 88 & 87 & 90 & 86 \\ 15 & 94 & -- & -- & -- & --\end{array}$

mean dur:

$4 \quad 3$

(at exit /conditional on exit)

1st decile at dur:

1st quartile at:

median at dur:

3rd quartile at:

$\begin{array}{lllll}1 & 1 & 1 & 1 & 1 \\ 2 & 2 & 2 & 1 & 1 \\ 3 & 3 & 3 & 3 & 3 \\ 7 & 5 & 6 & 5 & 6\end{array}$

mean dur:

44

(of all episodes / truncated after 15 years) 
$=>$ Table 23: Cumulative percent no longer in a consensual union, by time since union formation

$\begin{array}{cccccc}\text { duration in years } & \text { GDR } & \text { Hungary } & \text { Czech R } & \text { Slovenia } & \text { Latvia } \\ & (1984-89) & (1988-93) & (1992-97) & (1989-95) & (1989-95)\end{array}$

$\begin{array}{lccccc}0 & 0 & 0 & 0 & 0 & 0 \\ 1 & 32 & 34 & 30 & 24 & 40 \\ 2 & 56 & 55 & 59 & 42 & 61 \\ 3 & 68 & 67 & 70 & 56 & 70 \\ 4 & 78 & 74 & 76 & 61 & 77 \\ 5 & 82 & 79 & 79 & 66 & 82 \\ 7 & 87 & 84 & 85 & 73 & 86 \\ 10 & 90 & 90 & -- & 80 & 91 \\ 15 & -- & -- & -- & 83 & --\end{array}$

mean dur:

2

3

(at exit /conditional on exit)

1st decile at dur:

1st quartile at:

median at dur:

3rd quartile at:

$\begin{array}{lll}1 & 1 & 1 \\ 1 & 1 & 1 \\ 2 & 2 & 2 \\ 4 & 5 & 4\end{array}$

$1 \quad 1$

21

32

mean dur:

3

8

4

(of all episodes / truncated after 15 years)

duration in years

Lithuania Poland

(1989-95) (1986-91)

$\begin{array}{lcc}0 & 0 & 0 \\ 1 & 53 & 26 \\ 2 & 68 & 41 \\ 3 & 77 & 47 \\ 4 & 82 & 49 \\ 5 & 87 & 51 \\ 7 & 92 & -- \\ 10 & 93 & -- \\ 15 & -- & --\end{array}$

mean dur:

(at exit /conditional on exit)

1st decile at dur:

1st quartile at:

median at dur:

3rd quartile at:

41

47

49

51

$--$

mean dur:

(of all episodes / truncated after 15 years) 
Table 24: Cumulative percent separated, by time since union formation, for unions begun as a cohabitation (without censoring at marriage formation)
duration in years
Sweden Norway Finland France USA
$(1985-93) \quad(1983-89) \quad(1983-92) \quad(1988-94) \quad(1989-95)$

0
1
2
3
4
5
7
10
15
20

$\begin{array}{ccccc}0 & 0 & 0 & 0 & 0 \\ 8 & 8 & 6 & 8 & 25 \\ 18 & 16 & 12 & 15 & 38 \\ 26 & 22 & 18 & 20 & 46 \\ 32 & 27 & 24 & 25 & 51 \\ 37 & 30 & 27 & 29 & 55 \\ 43 & 34 & 32 & 36 & 60 \\ 49 & 39 & 36 & 42 & 66 \\ 55 & 45 & 42 & 48 & 72 \\ 59 & 50 & 44 & 54 & 76\end{array}$

mean dur:

5

6

5

6

4

(at union disruption / conditional on disruption)

1st decile at dur:

1st quartile at:

median at dur:

$2 \quad 2$

2

5

$2 \quad 1$

3rd quartile at:

1120

mean dur:

11

13

(of all unions / truncated after 20 years)

West

duration in years

Austria Germany Flanders Italy Spain

$\begin{array}{lllll}(1990-96) & (1986-92) & (1985-92) & (1990-95) & (1989-95)\end{array}$

$\begin{array}{lccccc}0 & 0 & 0 & 0 & 0 & 0 \\ 1 & 4 & 5 & 4 & 18 & 21 \\ 2 & 12 & 15 & 9 & 23 & 29 \\ 3 & 19 & 23 & 13 & 29 & 33 \\ 4 & 24 & 28 & 16 & 31 & 39 \\ 5 & 27 & 32 & 21 & 33 & 42 \\ 7 & 33 & 38 & 25 & 36 & 47 \\ 10 & 40 & 44 & 28 & 38 & 53 \\ 15 & 45 & 51 & 38 & 43 & 55 \\ 20 & 51 & 55 & -- & 49 & 58\end{array}$

mean dur:

$6 \quad 6$

5

4

(at union disruption / conditional on disruption)

1st decile at dur:

1st quartile at:

median at dur:

$\begin{array}{lllll}2 & 2 & 3 & 1 & 1\end{array}$

3rd quartile at:

20

32

mean dur:

13

(of all unions / truncated after 20 years)

12

$--$

13

11

0
21
29
33
39
42
47
53
55
58
4


1
2
--


Table 24: Cumulative percent separated, by time since union formation, for unions begun as a cohabitation (without censoring at marriage formation)
duration in years
GDR Hungary Czech R Slovenia Latvia
$(1984-89) \quad(1988-93) \quad(1992-97) \quad(1989-95) \quad(1989-95)$

$\begin{array}{cccccc}0 & 0 & 0 & 0 & 0 & 0 \\ 1 & 8 & 10 & 7 & 3 & 13 \\ 2 & 15 & 20 & 13 & 6 & 24 \\ 3 & 21 & 26 & 19 & 11 & 29 \\ 4 & 27 & 32 & 23 & 13 & 37 \\ 5 & 30 & 35 & 26 & 14 & 42 \\ 7 & 37 & 40 & 29 & 16 & 47 \\ 10 & 41 & 46 & 32 & 20 & 53 \\ 15 & 49 & 53 & 39 & 22 & 57 \\ 20 & -- & 58 & 45 & 25 & 59\end{array}$

mean dur:

6

6

6

4

(at union disruption / conditional on disruption)

1st decile at dur:

1st quartile at:

median at dur:

3rd quartile at:

$2 \quad 1$

$\begin{array}{lll}2 & 3 & 1\end{array}$

mean dur:

$\begin{array}{cc}4 & 3 \\ 16 & 14\end{array}$

(of all unions / truncated after 20 years)

12

14

17

11

duration in years

Lithuania Poland

(1989-95) (1986-91)

$\begin{array}{lcc}0 & 0 & 0 \\ 1 & 8 & 5 \\ 2 & 15 & 10 \\ 3 & 20 & 12 \\ 4 & 25 & 12 \\ 5 & 32 & 15 \\ 7 & 41 & 15 \\ 10 & 44 & 17 \\ 15 & 55 & 21 \\ 20 & 58 & 30\end{array}$

mean dur:

6

9

(at union disruption / conditional on disruption)

1st decile at dur:

$2 \quad 2$

1st quartile at:

420

median at dur:

13

3rd quartile at:

mean dur:

12

17

(of all unions / truncated after 20 years) 
Table 25: Cumulative percent separated, by time since union formation, for unions begun as a marriage

$\begin{array}{lccccc}\text { duration in years } & \text { Sweden } & \text { Norway } & \text { Finland } & \text { France } & \text { USA } \\ & (1985-93) & (1983-89) & (1983-92) & (1988-94) & (1989-95)\end{array}$

$\begin{array}{lccccc}0 & 0 & 0 & 0 & 0 & 0 \\ 1 & 3 & 0 & 1 & 1 & 5 \\ 2 & 5 & 2 & 3 & 2 & 10 \\ 3 & 5 & 5 & 5 & 3 & 14 \\ 4 & 5 & 9 & 8 & 5 & 17 \\ 5 & 5 & 10 & 10 & 7 & 20 \\ 7 & 8 & 13 & 12 & 8 & 25 \\ 10 & 14 & 17 & 17 & 11 & 31 \\ 15 & 20 & 23 & 21 & 16 & 38 \\ 20 & 24 & 28 & 25 & 19 & 44 \\ \text { mean dur: } & 9 & 9 & 8 & 9 & 7 \\ \text { (at union disruption /conditional on disruption) } & & & \\ & & & & & \\ \text { 1st decile at dur: } & 8 & 5 & 5 & 9 & 2 \\ \text { 1st quartile at: } & -- & 17 & 20 & -- & 7 \\ \text { median at dur: } & -- & -- & -- & -- & -- \\ \text { 3rd quartile at: } & -- & -- & -- & -- & - \\ & & & & & \\ \text { mean dur: } & 17 & 17 & 17 & 18 & 14 \\ \text { (of all unions / truncated after } & 20 \text { years) } & & & & \end{array}$

$\begin{array}{cccccc} & \text { West } & & \\ \text { duration in years } & \text { Austria } & \text { Germany } & \text { Flanders } & \text { Italy } & \text { Spain } \\ & (1990-96) & (1986-92) & (1985-92) & (1990-95) & (1989-95)\end{array}$

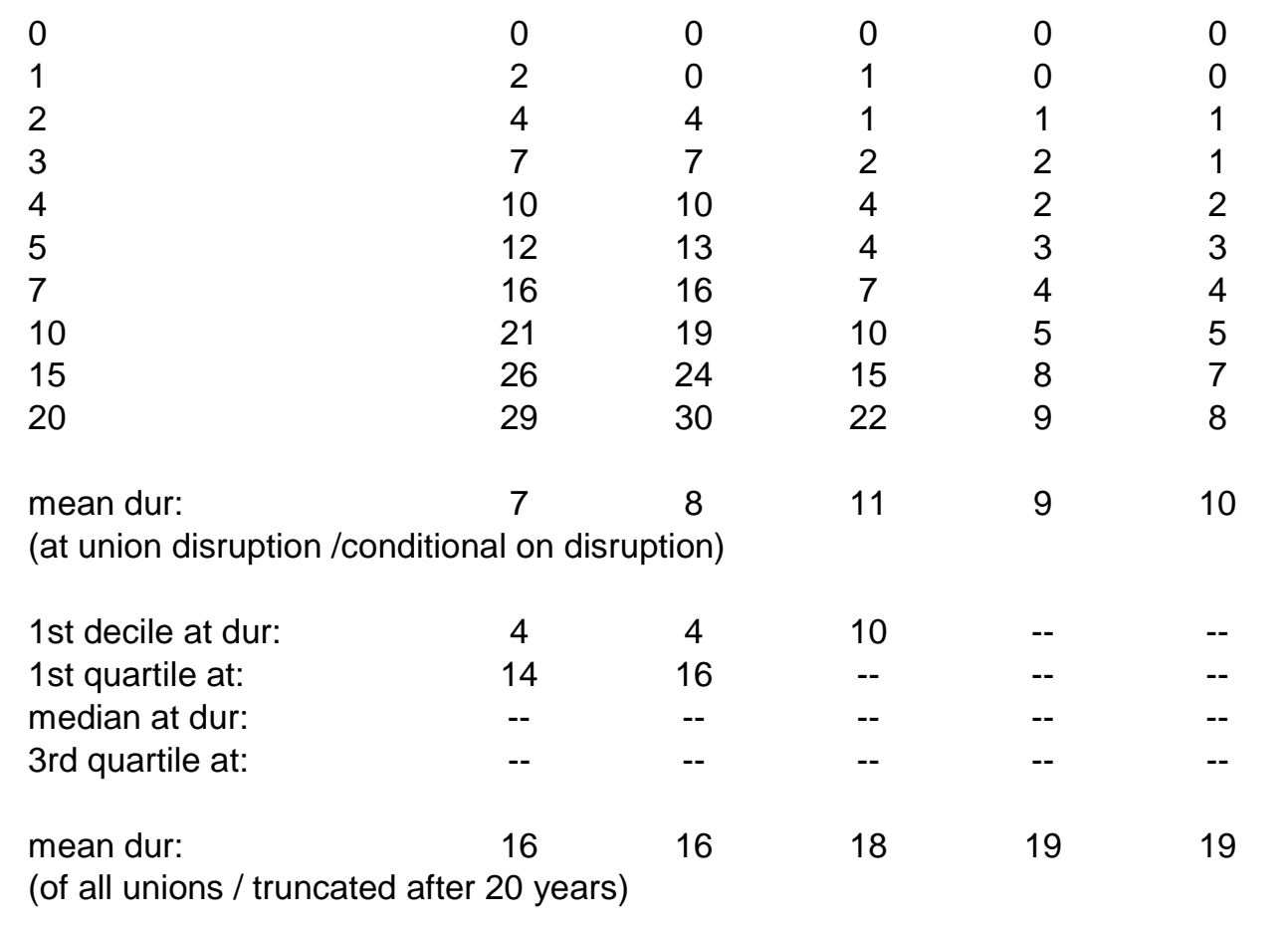


Table 25: Cumulative percent separated, by time since union formation, for unions begun as a marriage

$\begin{array}{cccccc}\text { duration in years } & \text { GDR } & \text { Hungary } & \text { Czech R } & \text { Slovenia } & \text { Latvia } \\ & (1984-89) & (1988-93) & (1992-97) & (1989-95) & (1989-95)\end{array}$

\begin{tabular}{|c|c|c|c|c|c|}
\hline 0 & 0 & 0 & 0 & 0 & 0 \\
\hline 1 & 1 & 2 & 1 & 1 & 2 \\
\hline 2 & 3 & 4 & 3 & 1 & 6 \\
\hline 3 & 5 & 6 & 6 & 1 & 10 \\
\hline 4 & 7 & 7 & 7 & 2 & 15 \\
\hline 5 & 10 & 9 & 10 & 2 & 20 \\
\hline 7 & 13 & 12 & 14 & 3 & 24 \\
\hline 10 & 19 & 16 & 18 & 5 & 29 \\
\hline 15 & 24 & 20 & 26 & 7 & 35 \\
\hline 20 & -- & 24 & 31 & 9 & 38 \\
\hline & \multicolumn{4}{|c|}{ (at union disruption /conditional on disruption) } & 7 \\
\hline 1st decile at dur: & 5 & 6 & 5 & -- & 3 \\
\hline 1st quartile at: & 16 & -- & 13 & -- & 8 \\
\hline median at dur: & -- & -- & -- & -- & -- \\
\hline 3rd quartile at: & -- & -- & -- & -- & -- \\
\hline mean dur: & -- & 17 & 16 & 19 & 15 \\
\hline
\end{tabular}

$\begin{array}{lcc}\text { duration in years } & \text { Lithuania } & \text { Poland } \\ (1989-95) & (1986-91)\end{array}$

$\begin{array}{lcc}0 & 0 & 0 \\ 1 & 1 & 1 \\ 2 & 2 & 2 \\ 3 & 3 & 2 \\ 4 & 6 & 4 \\ 5 & 9 & 4 \\ 7 & 11 & 5 \\ 10 & 15 & 6 \\ 15 & 19 & 8 \\ 20 & 23 & 11\end{array}$

mean dur: $\quad 8 \quad 9$

(at union disruption /conditional on disruption)

1st decile at dur: $\quad 6 \quad 18$

1st quartile at: $\quad$-- $\quad$--

median at dur: $\quad$-- $\quad$--

3rd quartile at: $\quad--$

mean dur: $\quad 17 \quad 19$

(of all unions / truncated after 20 years) 
=> Table 26: Cumulative percent separated, by time since union formation, all unions

$\begin{array}{lccccc}\text { duration in years } & \text { Sweden } & \text { Norway } & \text { Finland } & \text { France } & \text { USA } \\ & (1985-93) & (1983-89) & (1983-92) & (1988-94) & (1989-95)\end{array}$

$\begin{array}{lccccc}0 & 0 & 0 & 0 & 0 & 0 \\ 1 & 8 & 7 & 5 & 7 & 19 \\ 2 & 17 & 14 & 11 & 13 & 30 \\ 3 & 26 & 20 & 16 & 18 & 36 \\ 4 & 32 & 24 & 21 & 22 & 41 \\ 5 & 36 & 27 & 25 & 26 & 44 \\ 7 & 42 & 31 & 29 & 31 & 49 \\ 10 & 48 & 35 & 34 & 36 & 55 \\ 15 & 54 & 40 & 38 & 41 & 61 \\ 20 & 58 & 45 & 41 & 45 & 65 \\ \text { mean dur: } & 5 & 6 & 6 & 6 & 5 \\ \text { (at union disruption / conditional on disruption) } & & & \\ & & & & & \\ \text { 1st decile at dur: } & 2 & 2 & 2 & 2 & 1 \\ \text { 1st quartile at: } & 3 & 5 & 5 & 5 & 2 \\ \text { median at dur: } & 12 & -- & -- & -- & 8 \\ \text { 3rd quartile at: } & -- & -- & -- & -- & - \\ & & & & & \\ \text { mean dur: } & 12 & 14 & 14 & 14 & 10 \\ \text { (of all unions / truncated after } 20 \text { years) } & & & & \end{array}$

$\begin{array}{cccccc} & \text { West } & & \\ \text { duration in years } & \text { Austria } & \text { Germany } & \text { Flanders } & \text { Italy } & \text { Spain } \\ & (1990-96) & (1986-92) & (1985-92) & (1990-95) & (1989-95)\end{array}$

$\begin{array}{lccccc}0 & 0 & 0 & 0 & 0 & 0 \\ 1 & 4 & 4 & 2 & 4 & 6 \\ 2 & 11 & 12 & 4 & 5 & 9 \\ 3 & 17 & 19 & 6 & 7 & 10 \\ 4 & 21 & 24 & 8 & 8 & 12 \\ 5 & 23 & 27 & 10 & 9 & 14 \\ 7 & 29 & 32 & 13 & 10 & 16 \\ 10 & 35 & 37 & 16 & 12 & 18 \\ 15 & 40 & 43 & 21 & 14 & 20 \\ 20 & 44 & 47 & 27 & 16 & 21 \\ \text { mean dur: } & & & & & \\ \text { (at union disruption / } & 6 & 6 & 9 & 6 & 5 \\ \text { conditional on disruption) } & & & & & \\ \text { 1st decile at dur: } & & & & & \\ \text { 1st quartile at: } & 2 & 2 & 5 & 6 & 3 \\ \text { median at dur: } & 6 & 5 & 19 & -- & -- \\ \text { 3rd quartile at: } & -- & -- & -- & -- & - \\ & -- & -- & -- & -- & \\ \text { mean dur: } & & & & & \\ \text { (of all unions / truncated after } & 14 & 13 & 17 & 18 & 17\end{array}$


=> Table 26: Cumulative percent separated, by time since union formation, all unions

$\begin{array}{cccccc}\text { duration in years } & \text { GDR } & \text { Hungary } & \text { Czech R } & \text { Slovenia } & \text { Latvia } \\ (1984-89) & (1988-93) & (1992-97) & (1989-95) & (1989-95)\end{array}$

$\begin{array}{lccccc}0 & 0 & 0 & 0 & 0 & 0 \\ 1 & 5 & 5 & 5 & 2 & 9 \\ 2 & 10 & 10 & 9 & 4 & 16 \\ 3 & 14 & 14 & 13 & 7 & 21 \\ 4 & 18 & 16 & 16 & 8 & 28 \\ 5 & 21 & 19 & 19 & 9 & 33 \\ 7 & 26 & 22 & 23 & 11 & 37 \\ 10 & 31 & 26 & 26 & 13 & 42 \\ 15 & 37 & 30 & 33 & 15 & 47 \\ 20 & -- & 34 & 38 & 17 & 50 \\ \text { mean dur: } & 8 & 6 & 7 & 6 & 5 \\ \text { (at union disruption / conditional on disruption) } & & & \\ & & & & & \\ \text { 1st decile at dur: } & 2 & 2 & 3 & 6 & 2 \\ \text { 1st quartile at: } & 7 & 9 & 8 & -- & 4 \\ \text { median at dur: } & 19 & -- & -- & -- & 20 \\ \text { 3rd quartile at: } & -- & -- & -- & -- & - \\ & & & & & \\ \text { mean dur: } & 14 & 15 & 15 & 18 & 13 \\ \text { (of all unions / truncated after } 20 \text { years) } & & & & \end{array}$

$\begin{array}{lcc}0 & 0 & 0 \\ 1 & 3 & 1 \\ 2 & 5 & 3 \\ 3 & 8 & 3 \\ 4 & 11 & 4 \\ 5 & 14 & 5 \\ 7 & 18 & 6 \\ 10 & 21 & 7 \\ 15 & 26 & 9 \\ 20 & 30 & 12 \\ & & \\ \text { mean dur: } & 7 & 9 \\ \text { (at union disruption / conditional on disruption) } \\ \text { 1st decile at dur: } & 4 & 16 \\ \text { 1st quartile at: } & 14 & -- \\ \text { median at dur: } & -- & -- \\ \text { 3rd quartile at: } & -- & -- \\ & & \\ \text { mean dur: } & 16 & 19 \\ \text { (of all unions / truncated after } & 20 \text { years) } & \end{array}$


Table 27: Cumulative percent separated, by time since marriage formation, all marriages

$\begin{array}{lccccc}\text { duration in years } & \text { Sweden } & \text { Norway } & \text { Finland } & \text { France } & \text { USA } \\ & (1985-93) & (1983-89) & (1983-92) & (1988-94) & (1989-95)\end{array}$

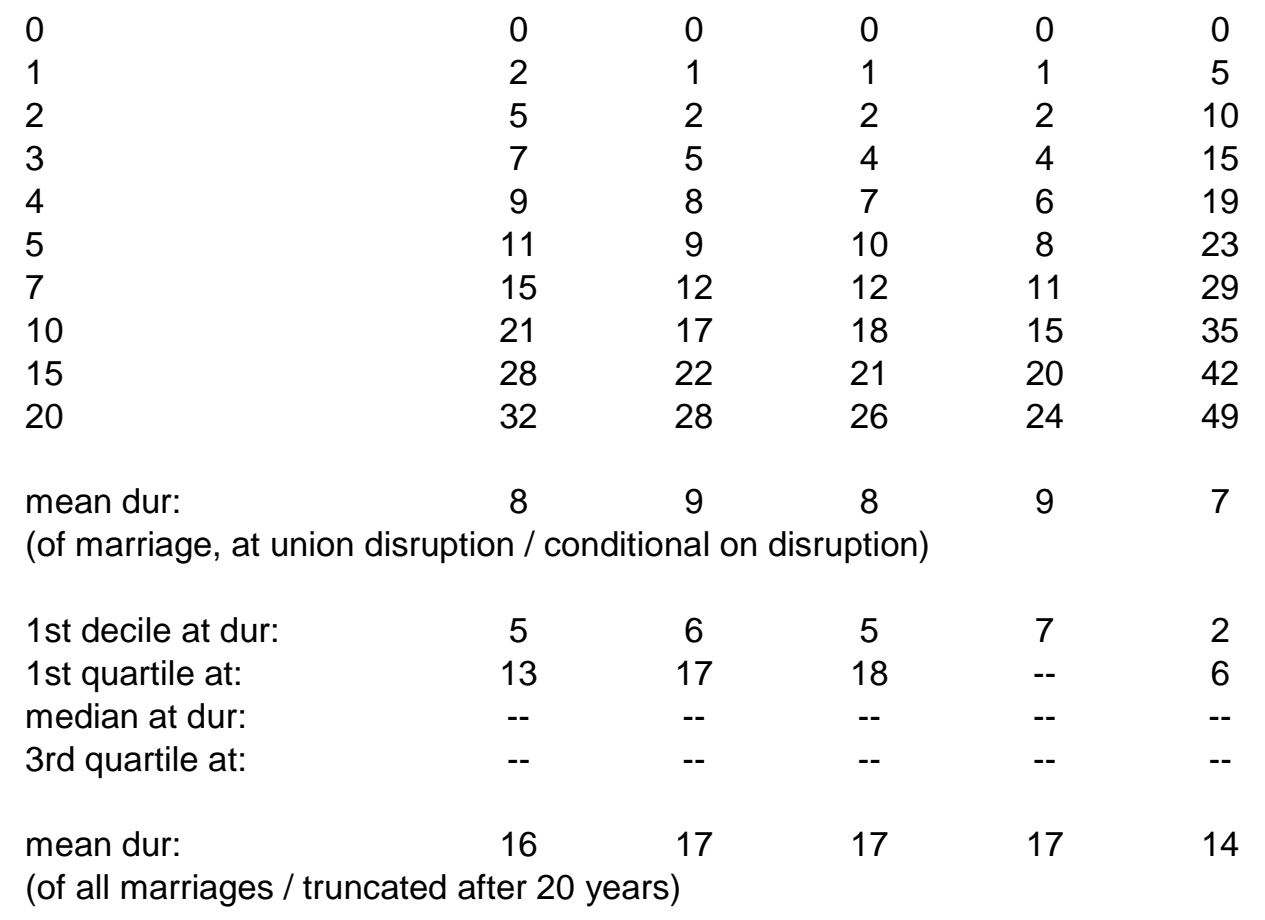

$\begin{array}{lccccc}0 & 0 & 0 & 0 & 0 & 0 \\ 1 & 1 & 1 & 1 & 0 & 0 \\ 2 & 3 & 4 & 2 & 1 & 1 \\ 3 & 5 & 7 & 3 & 2 & 2 \\ 4 & 8 & 10 & 4 & 2 & 2 \\ 5 & 11 & 12 & 5 & 3 & 4 \\ 7 & 14 & 17 & 7 & 4 & 5 \\ 10 & 19 & 21 & 11 & 5 & 6 \\ 15 & 25 & 27 & 16 & 8 & 8 \\ 20 & 30 & 33 & 23 & 10 & 9 \\ \text { mean dur: } & 8 & 8 & 11 & 9 & 9 \\ \text { (of marriage, at union disruption / conditional on disruption) } & & \\ \text { 1st decile at dur: } & 5 & 4 & 9 & 19 & -- \\ \text { 1st quartile at: } & 14 & 13 & -- & -- & -- \\ \text { median at dur: } & -- & -- & -- & -- & -- \\ \text { 3rd quartile at: } & -- & -- & -- & -- & \\ \text { mean dur: } & 16 & 16 & 18 & 19 & 19 \\ \text { (of all marriages / truncated after } 20 \text { years) } & & & & \end{array}$


Table 27: Cumulative percent separated, by time since marriage formation, all marriages

$\begin{array}{cccccc}\text { duration in years } & \text { GDR } & \text { Hungary } & \text { Czech R } & \text { Slovenia } & \text { Latvia } \\ & (1984-89) & (1988-93) & (1992-97) & (1989-95) & (1989-95)\end{array}$

\begin{tabular}{|c|c|c|c|c|c|}
\hline 0 & 0 & 0 & 0 & 0 & 0 \\
\hline 1 & 1 & 2 & 2 & 1 & 3 \\
\hline 2 & 3 & 4 & 4 & 1 & 8 \\
\hline 3 & 5 & 6 & 8 & 2 & 12 \\
\hline 4 & 8 & 7 & 10 & 2 & 17 \\
\hline 5 & 11 & 9 & 12 & 2 & 2 \\
\hline 7 & 16 & 13 & 16 & 4 & 2 \\
\hline 10 & 22 & 17 & 20 & 6 & 2 \\
\hline 15 & 28 & 21 & 28 & 8 & 35 \\
\hline 20 & -- & 25 & 34 & 10 & 3 \\
\hline mean dur: & -- & 8 & 9 & 9 & 7 \\
\hline \multicolumn{6}{|c|}{ (of marriage, at union disruption / conditional on disruption) } \\
\hline 1st decile at dur: & 5 & 6 & 4 & 19 & 3 \\
\hline 1st quartile at: & 12 & 19 & 13 & -- & 7 \\
\hline median at dur: & -- & -- & -- & -- & - \\
\hline 3rd quartile at: & -- & -- & -- & -- & \\
\hline mean dur: & -- & 17 & 16 & 19 & ( \\
\hline
\end{tabular}

(of all marriages / truncated after 20 years)
duration in years

$$
\begin{array}{cc}
\text { Lithuania } & \text { Poland } \\
(1989-95) & (1986-91)
\end{array}
$$

\begin{tabular}{lcc}
0 & 0 & 0 \\
1 & 1 & 1 \\
2 & 3 & 2 \\
3 & 4 & 3 \\
4 & 7 & 4 \\
5 & 10 & 5 \\
7 & 13 & 5 \\
10 & 17 & 7 \\
15 & 21 & 9 \\
20 & 25 & 12 \\
& & \\
mean dur: & 8 & 9 \\
(of marriage, at union disruption / conditional on disruption) \\
1st decile at dur: & 5 & 17 \\
1st quartile at: & 19 & -- \\
median at dur: & -- & -- \\
3rd quartile at: & -- & -- \\
& \multicolumn{2}{|c}{} \\
mean dur: & 17 & 19 \\
(of all marriages / truncated after 20 years)
\end{tabular}


Table 28: Cumulative percent separated, by time since union formation/entry into parenthood, unions of parents

$\begin{array}{lccccc}\text { duration in years } & \text { Sweden } & \text { Norway } & \text { Finland } & \text { France } & \text { USA } \\ & (1985-93) & (1983-89) & (1983-92) & (1988-94) & (1989-95)\end{array}$

\begin{tabular}{|c|c|c|c|c|c|}
\hline 0 & 0 & 0 & 0 & 0 & 0 \\
\hline 1 & 4 & 4 & 2 & 2 & 14 \\
\hline 2 & 8 & 8 & 4 & 4 & 21 \\
\hline 3 & 12 & 11 & 8 & 7 & 27 \\
\hline 4 & 16 & 14 & 12 & 10 & 31 \\
\hline 5 & 20 & 16 & 14 & 12 & 34 \\
\hline 7 & 24 & 20 & 17 & 16 & 41 \\
\hline 10 & 29 & 24 & 22 & 20 & 46 \\
\hline 15 & 36 & 30 & 26 & 26 & 53 \\
\hline 20 & 43 & 35 & 30 & 31 & 59 \\
\hline \multicolumn{6}{|c|}{ (at union disruption / conditional on disruption) } \\
\hline 1st decile at dur: & 3 & 3 & 4 & 4 & 1 \\
\hline 1st quartile at: & 8 & 11 & 15 & 14 & 3 \\
\hline median at dur: & -- & -- & -- & -- & 13 \\
\hline 3rd quartile at: & -- & -- & -- & -- & -- \\
\hline $\begin{array}{l}\text { mean dur: } \\
\text { (of all episodes / }\end{array}$ & 15 & 16 & 16 & 16 & 12 \\
\hline
\end{tabular}

\begin{tabular}{|c|c|c|c|c|c|}
\hline & & West & & & \\
\hline duration in & $\begin{array}{c}\text { Austria } \\
(1990-96)\end{array}$ & $\begin{array}{c}\text { Germany } \\
(1986-92)\end{array}$ & $\begin{array}{c}\text { Flanders } \\
(1985-92)\end{array}$ & $\begin{array}{c}\text { Italy } \\
(1990-95)\end{array}$ & $\begin{array}{c}\text { Spain } \\
(1989-95)\end{array}$ \\
\hline
\end{tabular}

\begin{tabular}{|c|c|c|c|c|c|}
\hline 0 & 0 & 0 & 0 & 0 & 0 \\
\hline 1 & 2 & 2 & 1 & 0 & 2 \\
\hline 2 & 4 & 6 & 2 & 1 & 3 \\
\hline 3 & 8 & 8 & 3 & 2 & 4 \\
\hline 4 & 12 & 11 & 4 & 2 & 5 \\
\hline 5 & 14 & 14 & 5 & 3 & 6 \\
\hline 7 & 18 & 17 & 7 & 4 & 7 \\
\hline 10 & 23 & 22 & 12 & 6 & 8 \\
\hline 15 & 28 & 30 & 16 & 8 & 10 \\
\hline 20 & 33 & 35 & 23 & 10 & 12 \\
\hline \multicolumn{6}{|c|}{ (at union disruption / conditional on disruption) } \\
\hline 1st decile at dur: & 4 & 4 & 9 & 20 & 13 \\
\hline 1st quartile at: & 12 & 12 & -- & -- & -- \\
\hline median at dur: & -- & -- & -- & -- & -- \\
\hline 3rd quartile at: & -- & -- & -- & -- & -- \\
\hline $\begin{array}{l}\text { mean dur: } \\
\text { (of all episodes / }\end{array}$ & $\begin{array}{l}16 \\
\text { er } 20\end{array}$ & 16 & 18 & 19 & 18 \\
\hline
\end{tabular}


Table 28: Cumulative percent separated, by time since union formation/entry into parenthood, unions of parents

$\begin{array}{cccccc}\text { duration in years } & \text { GDR } & \text { Hungary } & \text { Czech R } & \text { Slovenia } & \text { Latvia } \\ & (1984-89) & (1988-93) & (1992-97) & (1989-95) & (1989-95)\end{array}$

\begin{tabular}{|c|c|c|c|c|c|}
\hline 0 & 0 & 0 & 0 & 0 & 0 \\
\hline 1 & 4 & 3 & 3 & 1 & 5 \\
\hline 2 & 7 & 5 & 8 & 2 & 11 \\
\hline 3 & 11 & 7 & 12 & 3 & 16 \\
\hline 4 & 14 & 9 & 13 & 4 & 21 \\
\hline 5 & 17 & 11 & 16 & 5 & 24 \\
\hline 7 & 21 & 14 & 19 & 6 & 28 \\
\hline 10 & 26 & 18 & 22 & 9 & 32 \\
\hline 15 & 32 & 21 & 29 & 11 & 38 \\
\hline 20 & -- & 26 & 35 & 13 & 42 \\
\hline \multicolumn{5}{|c|}{ (at union disruption / conditional on disruption) } & 6 \\
\hline 1st decile at dur: & 3 & 5 & 3 & 12 & 2 \\
\hline 1st quartile at: & 9 & 18 & 12 & -- & 6 \\
\hline median at dur: & -- & -- & -- & -- & - \\
\hline 3rd quartile at: & -- & -- & -- & -- & - \\
\hline mean dur: & -- & 17 & 16 & 18 & 14 \\
\hline
\end{tabular}

(of all episodes / truncated after 20 years)

$$
\begin{array}{lcc}
\text { duration in years } & \text { Lithuania } & \text { Poland } \\
& (1989-95) & (1986-91)
\end{array}
$$

$\begin{array}{lcc}0 & 0 & 0 \\ 1 & 2 & 1 \\ 2 & 4 & 2 \\ 3 & 7 & 3 \\ 4 & 9 & 4 \\ 5 & 11 & 4 \\ 7 & 14 & 4 \\ 10 & 18 & 6 \\ 15 & 22 & 8 \\ 20 & 26 & 11\end{array}$

mean dur: $\quad 8 \quad 9$

(at union disruption / conditional on disruption)

1st decile at dur: $\quad 5 \quad 18$

1st quartile at: $\quad 18 \quad--$

median at dur: $\quad--\quad$

3rd quartile at: $\quad--\quad \quad--$

mean dur: $\quad 17 \quad 19$

(of all episodes / truncated after 20 years) 
Table 29: Percent of time spent in different family types at ages 15-39 years / Men

\begin{tabular}{|c|c|c|c|}
\hline $\begin{array}{c}\text { Sweden } \\
(1978-93)\end{array}$ & $\begin{array}{c}\text { Norway } \\
(1974-89)\end{array}$ & $\begin{array}{l}\text { Finland } \\
(1983-92)\end{array}$ & $\begin{array}{r}\text { Fra } \\
(1988\end{array}$ \\
\hline 23 & 28 & 32 & \\
\hline 15 & 12 & 13 & \\
\hline 0 & 1 & 1 & \\
\hline 14 & 7 & 10 & \\
\hline 11 & 3 & 4 & \\
\hline 3 & 6 & 6 & \\
\hline 26 & 40 & 31 & \\
\hline 5 & 2 & 2 & \\
\hline 3 & 3 & 2 & \\
\hline 54 & 55 & 50 & \\
\hline 37 & 43 & 34 & \\
\hline 17 & 13 & 16 & \\
\hline 41 & 46 & 37 & \\
\hline 37 & 43 & 34 & \\
\hline 4 & 3 & 2 & \\
\hline
\end{tabular}

In parental home /no family

Single and never in union /no child

Single and never in union /parent

In consensual union /no child

In consensual union /parent

In marriage /no child

In marriage /parent

Single after family disruption /no child

Single after family disruption /parent

Time in union

-in union as a parent

-in union but no child

Time as parent

-as parent in union

-as parent out of union

West

Austria Germany Flanders Italy Spain

(1990-96) (1986-92) (1985-92) (1990-95) (1989-95)

In parental home /no family

32

$\begin{array}{cccc}34 & 40 & 52 & 48 \\ 19 & 5 & 8 & 4 \\ 1 & 0 & 0 & 0 \\ 7 & 4 & 1 & 3 \\ 2 & 2 & 0 & 1 \\ 7 & 10 & 8 & 7 \\ 23 & 37 & 29 & 35 \\ 4 & 2 & 2 & 2 \\ 3 & 2 & 0 & 1\end{array}$

Time in union

39

51

38

46

-in union as a parent

25

38

29

USA

(NA)

-in union but no child

35

14

13

36

Time as parent

40

$9 \quad 10$

-as parent in union

35

25

-as parent out of union

3

3

30
29
1

37

36 
Table 29: Percent of time spent in different family types at ages 15-39 years / Men

\begin{tabular}{|c|c|c|c|c|}
\hline $\begin{array}{l}\text { GDR } \\
\text { (NA) }\end{array}$ & $\begin{array}{c}\text { Hungary } \\
(1988-93)\end{array}$ & $\begin{array}{c}\text { Czech R } \\
\text { (NA) }\end{array}$ & $\begin{array}{c}\text { Slovenia } \\
(1989-95)\end{array}$ & $\begin{array}{c}\text { Latvia } \\
(1989-95)\end{array}$ \\
\hline & 41 & & 30 & 32 \\
\hline & 2 & & 10 & 4 \\
\hline & 0 & & 0 & 0 \\
\hline & 2 & & 4 & 3 \\
\hline & 1 & & 6 & 2 \\
\hline & 6 & & 4 & 5 \\
\hline & 43 & & 43 & 48 \\
\hline & 2 & & 1 & 2 \\
\hline & 2 & & 1 & 4 \\
\hline & 52 & & 57 & 58 \\
\hline & 44 & & 49 & 50 \\
\hline & 8 & & 8 & 8 \\
\hline & 46 & & 50 & 54 \\
\hline & 44 & & 49 & 50 \\
\hline & 2 & & 2 & 4 \\
\hline
\end{tabular}

In parental home /no family

Single and never in union /no child

single and never in union /parent

in consensual union /no child

in consensual union /parent

in marriage /no child

in marriage /parent

single after family disruption /no child

single after family disruption /parent

time in union

-in union as a parent

-in union but no child

time as parent

-as parent in union

-as parent out of union

Lithuania Poland
(1989-95)

in parental home /no family $\quad 30$

single and never in union /no child 8

single and never in union /parent 0

in consensual union /no child 2

in consensual union /parent 1

in marriage /no child 8

in marriage /parent 48

single after family disruption /no child $\quad 1$

single after family disruption /parent 3

time in union $\quad 58$

-in union as a parent $\quad 49$

-in union but no child 9

time as parent 52

-as parent in union $\quad 49$

-as parent out of union 3 
Table 29: Percent of time spent in different family types at ages 15-39 years / Women

\begin{tabular}{|c|c|c|c|c|c|}
\hline & $\begin{array}{l}\text { Sweden } \\
(1978-93)\end{array}$ & $\begin{array}{c}\text { Norway } \\
(1974-89)\end{array}$ & $\begin{array}{c}\text { Finland } \\
(1979-89)\end{array}$ & $\begin{array}{c}\text { France } \\
(1988-94)\end{array}$ & $\begin{array}{c}\text { USA } \\
(1989-95)\end{array}$ \\
\hline in parental home /no family & 17 & 21 & 22 & 26 & 19 \\
\hline single and never in union /no child & 12 & 9 & 11 & 7 & 12 \\
\hline single and never in union /parent & 1 & 1 & 1 & 1 & 3 \\
\hline in consensual union /no child & 14 & 6 & 9 & 9 & 4 \\
\hline in consensual union /parent & 13 & 4 & 4 & 6 & 4 \\
\hline in marriage /no child & 3 & 6 & 6 & 5 & 10 \\
\hline in marriage /parent & 31 & 47 & 41 & 38 & 34 \\
\hline single after family disruption / no child & 4 & 2 & 2 & 3 & 4 \\
\hline single after family disruption /parent & 6 & 4 & 4 & 4 & 8 \\
\hline time in union & 61 & 64 & 60 & 58 & 53 \\
\hline -in union as a parent & 44 & 51 & 45 & 44 & 39 \\
\hline -in union but no child & 18 & 12 & 15 & 15 & 14 \\
\hline time as parent & 50 & 56 & 50 & 50 & 50 \\
\hline -as parent in union & 44 & 51 & 45 & 44 & 39 \\
\hline \multirow[t]{2}{*}{-as parent out of union } & 6 & 5 & 5 & 6 & 11 \\
\hline & $\begin{array}{c}\text { Austria } \\
(1990-96)\end{array}$ & $\begin{array}{c}\text { West } \\
\text { Germany } \\
(1986-92)\end{array}$ & $\begin{array}{c}\text { Flanders } \\
(1985-92)\end{array}$ & $\begin{array}{c}\text { Italy } \\
(1990-95)\end{array}$ & $\begin{array}{c}\text { Spain } \\
(1989-95)\end{array}$ \\
\hline in parental home /no family & 23 & 27 & 31 & 44 & 38 \\
\hline single and never in union /no child & 7 & 13 & 5 & 4 & 3 \\
\hline single and never in union /parent & 2 & 1 & 0 & 0 & 0 \\
\hline in consensual union /no child & 7 & 6 & 4 & 1 & 2 \\
\hline in consensual union /parent & 6 & 3 & 1 & 1 & 1 \\
\hline in marriage /no child & 5 & 8 & 11 & 8 & 7 \\
\hline in marriage /parent & 43 & 32 & 44 & 41 & 46 \\
\hline single after family disruption / no child & 2 & 3 & 1 & 1 & 1 \\
\hline single after family disruption /parent & 5 & 5 & 3 & 1 & 2 \\
\hline time in union & 61 & 50 & 60 & 50 & 56 \\
\hline -in union as a parent & 48 & 35 & 45 & 42 & 47 \\
\hline -in union but no child & 12 & 15 & 15 & 9 & 9 \\
\hline time as parent & 56 & 42 & 48 & 43 & 49 \\
\hline -as parent in union & 48 & 35 & 45 & 42 & 47 \\
\hline -as parent out of union & 7 & 6 & 3 & 2 & 2 \\
\hline
\end{tabular}


Table 29: Percent of time spent in different family types at ages 15-39 years / Women

$\begin{array}{lcccc}\text { GDR } & \text { Hungary } & \text { Czech R } & \text { Slovenia } & \text { Latvia } \\ \text { (NA) } & (1988-93) & (1992-97) & (1989-95) & (1989-95)\end{array}$

in parental home /no family

single and never in union /no child

single and never in union /parent

in consensual union /no child

in consensual union /parent

in marriage /no child

(NA)

in marriage /parent

single after family disruption /no child

single after family disruption /parent

time in union

-in union as a parent

-in union but no child

time as parent

-as parent in union

-as parent out of union

$\begin{array}{cccc}26 & 25 & 27 & 25 \\ 2 & 1 & 3 & 3 \\ 1 & 1 & 1 & 1 \\ 2 & 3 & 3 & 3 \\ 3 & 4 & 6 & 4 \\ 7 & 5 & 3 & 5 \\ 54 & 55 & 54 & 48 \\ 1 & 1 & 0 & 2 \\ 5 & 5 & 3 & 9 \\ & & & \\ 65 & 67 & 66 & 61 \\ 56 & 59 & 60 & 53 \\ 9 & 8 & 6 & 8 \\ & & & \\ 62 & 65 & 64 & 63 \\ 56 & 59 & 60 & 53 \\ 6 & 6 & 4 & 10\end{array}$

Lithuania Poland

(1989-95) (1986-91)

in parental home /no family

single and never in union /no child

$26 \quad 30$

single and never in union /parent

in consensual union /no child

63

in consensual union /parent

in marriage /no child

in marriage /parent

single after family disruption /no child

single after family disruption /parent

time in union

-in union as a parent

-in union but no child

time as parent

-as parent in union

-as parent out of union

13

10

11

$6 \quad 6$

$52 \quad 53$

$1 \quad 0$

63

$60 \quad 60$

$53 \quad 54$

76

$60 \quad 60$

$53 \quad 54$

$6 \quad 6$ 
Table 30: Relative distribution of births (percent)

$\begin{array}{ccccc}\text { Sweden } & \text { Norway } & \text { Finland } & \text { France } & \text { USA } \\ (1987-93) & (1983-89) & (1983-89) & (1988-94) & (1989-95\end{array}$

children born to mother never in union children born after union disruption $==>$ children born to lone mother children born in marriage children born in a consensual union

children born to mother never in union children born after union disruption $==>$ children born to lone mother children born in marriage children born in a consensual union

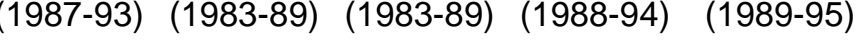

$\begin{array}{ccccc}1 & 5 & 1 & 3 & 11 \\ 3 & 2 & 1 & 7 & 6 \\ 5 & 7 & 3 & 10 & 17 \\ 51 & 71 & 85 & 68 & 72 \\ 45 & 22 & 13 & 23 & 11\end{array}$

West

Austria Germany Flanders Italy Spain (1990-96) (1986-92) (1985-92) (1990-95) (1989-95)

7
3
10
70
19

$\begin{array}{cc}4 & 0 \\ 2 & 1 \\ 6 & 1 \\ 83 & 94 \\ 11 & 4\end{array}$

$\begin{array}{cc}2 & 2 \\ 0 & 0 \\ 2 & 2 \\ 94 & 93 \\ 4 & 4\end{array}$


Table 30: Relative distribution of births (percent)

GDR Hungary Czech R Slovenia Latvia

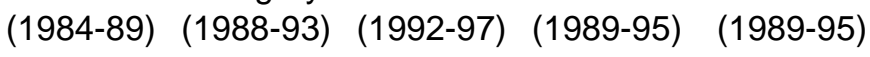

children born to mother never in union $15 \quad 2$

$3 \quad 2$

children born after union disruption

$==>$ children born to lone mother

children born in marriage

2

4

6

children born in a consensual union

90

6

5

4

140

$\begin{array}{cccc}4 & 6 & 11 \\ 69 & 78 & 79\end{array}$

79

Lithuania Poland

(1989-95) (1986-91)

children born to mother never in union children born after union disruption

$\begin{array}{cc}3 & 8 \\ 2 & 1 \\ 5 & 9 \\ 93 & 89 \\ 2 & 2\end{array}$

$==>$ children born to lone mother

children born in marriage

2

16

children born in a consensual union 
Table 31: Cumulative percent ever out of union, by age of child

age

0

1

2

3

4

6

9

12

15

age

0
1
2
3
4
6
9
12
15

15 $\begin{array}{ccccc}\text { Sweden } & \text { Norway } & \text { Finland } & \text { France } & \text { USA } \\ (1987-93) & (1983-89) & (1983-89) & (1988-94) & (1989-95)\end{array}$

$\begin{array}{ccccc}5 & 7 & 3 & 10 & 17 \\ 7 & 9 & 4 & 12 & 21 \\ 9 & 11 & 6 & 13 & 25 \\ 13 & 13 & 8 & 15 & 28 \\ 15 & 15 & 10 & 17 & 31 \\ 20 & 17 & 13 & 20 & 36 \\ 24 & 21 & 16 & 24 & 41 \\ 30 & 24 & 19 & 28 & 46 \\ 34 & 26 & 22 & 31 & 50\end{array}$

\section{West}

Austria Germany Flanders Italy Spain (1990-96) (1986-92) (1985-92) (1990-95) (1989-95)

$\begin{array}{ccccc}10 & 6 & 1 & 2 & 2 \\ 12 & 8 & 2 & 2 & 3 \\ 13 & 10 & 3 & 3 & 4 \\ 15 & 12 & 4 & 3 & 5 \\ 19 & 14 & 5 & 4 & 6 \\ 22 & 17 & 8 & 4 & 7 \\ 26 & 21 & 11 & 6 & 9 \\ 30 & 27 & 14 & 7 & 11 \\ 34 & 34 & 17 & 9 & 13\end{array}$


Table 31: Cumulative percent ever out of union, by age of child

age

0

1

2

3

4

6

9

12

15

age

0

1

2

3

4

6

9

12

15
GDR Hungary Czech R Slovenia Latvia (1984-89) (1988-93) (1992-97) (1989-95) (1989-95)

$\begin{array}{lcccc}18 & 3 & 4 & 6 & 11 \\ 19 & 5 & 6 & 7 & 15 \\ 22 & 6 & 9 & 7 & 18 \\ 25 & 8 & 11 & 8 & 22 \\ 28 & 10 & 13 & 8 & 25 \\ 32 & 13 & 16 & 10 & 29 \\ 37 & 17 & 20 & 12 & 35 \\ 42 & 21 & 24 & 14 & 40 \\ 46 & 24 & 29 & 15 & 44\end{array}$

Lithuania Poland

(1989-95) (1986-91)

$\begin{array}{cc}5 & 9 \\ 7 & 10 \\ 8 & 11 \\ 10 & 11 \\ 12 & 12 \\ 16 & 12 \\ 20 & 14 \\ 24 & 16 \\ 29 & 18\end{array}$


Table 32: Cumulative percent ever out of marriage, by age of child

age

0

1

2

3

4

6

9

12

15

age

0
1
2
3
4
6
9
12
15

Sweden Norway Finland France USA (1987-93) (1983-89) (1983-89) (1988-94) (1989-95)

$\begin{array}{lllll}49 & 29 & 15 & 32 & 28 \\ 50 & 29 & 16 & 33 & 30 \\ 51 & 30 & 17 & 34 & 33 \\ 52 & 31 & 19 & 35 & 35 \\ 53 & 33 & 20 & 36 & 37 \\ 55 & 34 & 22 & 37 & 41 \\ 57 & 37 & 25 & 40 & 45 \\ 60 & 40 & 27 & 42 & 49 \\ 61 & 42 & 30 & 44 & 53\end{array}$

\section{West}

Austria Germany Flanders Italy Spain (1990-96) (1986-92) (1985-92) (1990-95) (1989-95)

$\begin{array}{llccc}30 & 17 & 6 & 6 & 7 \\ 30 & 18 & 6 & 6 & 7 \\ 31 & 20 & 7 & 6 & 8 \\ 33 & 21 & 8 & 7 & 8 \\ 35 & 23 & 8 & 7 & 9 \\ 37 & 25 & 11 & 8 & 10 \\ 40 & 28 & 14 & 9 & 12 \\ 43 & 33 & 16 & 11 & 14 \\ 46 & 39 & 19 & 12 & 15\end{array}$


Table 32: Cumulative percent ever out of marriage, by age of child

age

0

1

2

3

4

6

9

12

15

age

0
1
2
3
4
6
9
12
15

GDR Hungary Czech R Slovenia Latvia (1984-89) (1988-93) (1992-97) (1989-95) (1989-95)

$\begin{array}{cc}33 & 10 \\ 33 & 11 \\ 35 & 11 \\ 37 & 13 \\ 40 & 15 \\ 43 & 18 \\ 47 & 21 \\ 50 & 25 \\ 54 & 28 \\ & \\ & \\ & \\ \text { ithuania } & \text { Poland } \\ \text { 989-95) } & \text { (1986-91) }\end{array}$

$\begin{array}{cc}7 & 11 \\ 8 & 12 \\ 10 & 13 \\ 12 & 13 \\ 14 & 13 \\ 17 & 14 \\ 21 & 16 \\ 25 & 18 \\ 29 & 20\end{array}$

21

24

27

29

32

35

40

45

48

\section{(1989-95) (1986-91)}


Table 33: Cumulative percent ever out of union, by age of child, for children born in a union

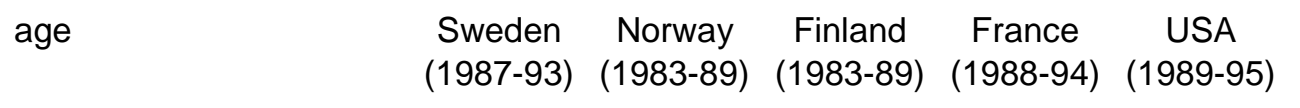

\begin{tabular}{|c|c|c|c|c|}
\hline 0 & 0 & 0 & 0 & 0 \\
\hline 1 & 2 & 2 & 1 & 2 \\
\hline 2 & 5 & 4 & 3 & 4 \\
\hline 3 & 9 & 6 & 6 & 6 \\
\hline 4 & 11 & 8 & 7 & 8 \\
\hline 6 & 16 & 10 & 10 & 12 \\
\hline 9 & 21 & 15 & 14 & 16 \\
\hline 12 & 26 & 18 & 17 & 20 \\
\hline 15 & 30 & 21 & 20 & 23 \\
\hline $\begin{array}{l}\text { mean age: } \\
\text { (at union disruptio }\end{array}$ & $\begin{array}{c}6 \\
\text { nal o }\end{array}$ & $\begin{array}{c}6 \\
\text { otion }\end{array}$ & 6 & 7 \\
\hline 1st decile at age: & 4 & 6 & 6 & 5 \\
\hline 1st quartile at: & 11 & -- & -- & -- \\
\hline median at age: & -- & -- & -- & -- \\
\hline 3rd quartile at: & -- & -- & -- & -- \\
\hline mean duration: & 12 & 13 & 13 & 13 \\
\hline
\end{tabular}

(of all episodes / truncated after 15 years)

\begin{tabular}{|c|c|c|c|c|c|}
\hline age & $\begin{array}{c}\text { Austria } \\
(1990-96)\end{array}$ & $\begin{array}{c}\text { West } \\
\text { Germany } \\
(1986-92)\end{array}$ & $\begin{array}{l}\text { Flanders } \\
(1985-92)\end{array}$ & $\begin{array}{c}\text { Italy } \\
(1990-95)\end{array}$ & $\begin{array}{c}\text { Spain } \\
(1989-95)\end{array}$ \\
\hline 0 & 0 & 0 & 0 & 0 & 0 \\
\hline 1 & 2 & 2 & 1 & 0 & 1 \\
\hline 2 & 3 & 4 & 2 & 1 & 2 \\
\hline 3 & 6 & 6 & 3 & 1 & 3 \\
\hline 4 & 9 & 8 & 4 & 2 & 4 \\
\hline 6 & 13 & 11 & 6 & 2 & 5 \\
\hline 9 & 18 & 16 & 10 & 4 & 7 \\
\hline 12 & 22 & 22 & 13 & 5 & 9 \\
\hline 15 & 26 & 29 & 15 & 7 & 10 \\
\hline $\begin{array}{l}\text { mean age: } \\
\text { (at union disruptio }\end{array}$ & $\begin{array}{c}7 \\
\text { litional on di }\end{array}$ & $\begin{array}{c}8 \\
\text { sruption) }\end{array}$ & 8 & 8 & 7 \\
\hline 1st decile at age: & 5 & 5 & 9 & -- & 14 \\
\hline 1st quartile at: & 14 & 14 & -- & -- & -- \\
\hline median at age: & -- & -- & -- & -- & -- \\
\hline 3rd quartile at: & -- & -- & -- & -- & -- \\
\hline $\begin{array}{l}\text { mean duration: } \\
\text { (of all episodes / th }\end{array}$ & $\begin{array}{c}13 \\
\text { after } 15 \text { ye }\end{array}$ & ars) & 14 & 15 & 14 \\
\hline
\end{tabular}


Table 33: Cumulative percent ever out of union, by age of child, for children born in a union

\begin{tabular}{|c|c|c|c|c|c|}
\hline age & $\begin{array}{c}\text { GDR } \\
(1984-89)\end{array}$ & $\begin{array}{l}\text { Hungary } \\
(1988-93)\end{array}$ & $\begin{array}{c}\text { Czech R } \\
(1992-97)\end{array}$ & $\begin{array}{c}\text { Slovenia } \\
(1989-95)\end{array}$ & $\begin{array}{c}\text { Latvia } \\
(1989-95)\end{array}$ \\
\hline 0 & 0 & 0 & 0 & 0 & 0 \\
\hline 1 & 2 & 1 & 2 & 1 & 5 \\
\hline 2 & 5 & 2 & 4 & 1 & 9 \\
\hline 3 & 8 & 4 & 7 & 2 & 13 \\
\hline 4 & 12 & 7 & 8 & 2 & 17 \\
\hline 6 & 17 & 10 & 12 & 4 & 21 \\
\hline 9 & 24 & 14 & 17 & 6 & 28 \\
\hline 12 & 29 & 18 & 21 & 8 & 33 \\
\hline 15 & 34 & 22 & 25 & 10 & 38 \\
\hline \multicolumn{6}{|c|}{ (at union disruption / conditional on disruption) } \\
\hline 1st decile at age: & 4 & 6 & 5 & 15 & 3 \\
\hline 1st quartile at: & 10 & -- & 15 & -- & 8 \\
\hline median at age: & -- & -- & -- & -- & -- \\
\hline 3rd quartile at: & -- & -- & -- & -- & -- \\
\hline $\begin{array}{l}\text { mean duration: } \\
\text { (of all episodes / }\end{array}$ & $\begin{array}{l}12 \\
\text { fter } 15 \text { y }\end{array}$ & 13 & 13 & 14 & 12 \\
\hline
\end{tabular}

\begin{tabular}{lcc} 
age & $\begin{array}{c}\text { Lithuania } \\
(1989-95)\end{array}$ & $\begin{array}{c}\text { Poland } \\
(1986-91)\end{array}$ \\
0 & 0 & 0 \\
1 & 2 & 1 \\
2 & 3 & 2 \\
3 & 5 & 2 \\
4 & 8 & 3 \\
6 & 11 & 4 \\
9 & 16 & 6 \\
12 & 20 & 8 \\
15 & 25 & 10 \\
& & \\
mean age: & 7 & 7 \\
(at union disruption / conditional on disruption) \\
1st decile at age: & \multicolumn{2}{c}{} \\
1st quartile at: & 5 & 14 \\
median at age: & -- & -- \\
3rd quartile at: & -- & -- \\
& \multicolumn{2}{c}{} \\
mean duration: & 13 & 14 \\
(of all episodes / truncated after 15 years)
\end{tabular}


Table 34: Cumulative percent ever out of union, by age of child, for children born in a consensual union

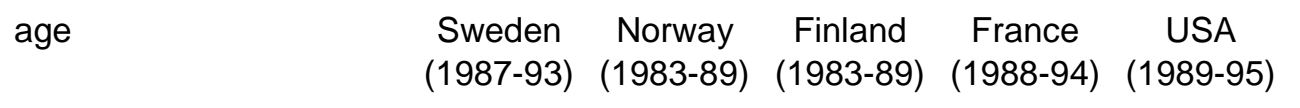

\begin{tabular}{|c|c|c|c|c|c|}
\hline 0 & 0 & 0 & 0 & 0 & 0 \\
\hline 1 & 4 & 8 & 4 & 5 & 18 \\
\hline 2 & 8 & 14 & 12 & 10 & 29 \\
\hline 3 & 13 & 18 & 19 & 16 & 39 \\
\hline 4 & 17 & 19 & 24 & 20 & 45 \\
\hline 6 & 23 & 21 & 29 & 27 & 56 \\
\hline 9 & 27 & 30 & 35 & 35 & 64 \\
\hline 12 & 32 & 32 & 37 & 49 & 70 \\
\hline 15 & 38 & 36 & 39 & 58 & 78 \\
\hline $\begin{array}{l}\text { mean age: } \\
\text { (at union disruptior }\end{array}$ & $\begin{array}{c}6 \\
\text { nal o }\end{array}$ & $\begin{array}{c}5 \\
\text { ption }\end{array}$ & 4 & 7 & 5 \\
\hline 1st decile at age: & 3 & 2 & 2 & 2 & 1 \\
\hline 1st quartile at: & 8 & 7 & 5 & 6 & 2 \\
\hline median at age: & -- & -- & -- & 13 & 6 \\
\hline 3rd quartile at: & -- & -- & -- & -- & 15 \\
\hline $\begin{array}{l}\text { mean duration: } \\
\text { (of all episodes / tr }\end{array}$ & $\begin{array}{l}12 \\
\operatorname{ter} 1\end{array}$ & 11 & 11 & 10 & 7 \\
\hline
\end{tabular}

$\begin{array}{lccccc}\text { age } & \begin{array}{c}\text { West } \\ \text { Austria } \\ (1990-96)\end{array} & \begin{array}{c}\text { Germany } \\ (1986-92)\end{array} & \begin{array}{c}\text { Flanders } \\ (1985-92)\end{array} & \begin{array}{c}\text { Italy } \\ (1990-95)\end{array} & \begin{array}{c}\text { Spain } \\ (1989-95)\end{array} \\ & & & & & \\ 0 & 0 & 0 & 0 & 0 & 0 \\ 1 & 6 & 4 & 4 & 0 & 9 \\ 2 & 8 & 11 & 10 & 2 & 18 \\ 3 & 13 & 16 & 15 & 2 & 24 \\ 4 & 20 & 20 & 25 & 4 & 26 \\ 6 & 26 & 27 & 25 & 7 & 31 \\ 9 & 31 & 37 & 34 & 7 & - \\ 12 & 37 & 53 & -- & -- & - \\ 15 & 43 & -- & -- & -- & -- \\ \text { mean age: } & 6 & -- & -- & -- & -- \\ \text { (at union disruption / conditional on disruption) } & & & \\ \text { 1st decile at age: } & & & & & \\ \text { 1st quartile at: } & 3 & 2 & 2 & -- & 2 \\ \text { median at age: } & 6 & 6 & 4 & -- & 4 \\ \text { 3rd quartile at: } & -- & 11 & -- & -- & - \\ \text { mean duration: } & -- & -- & -- & -- & - \\ \text { (of all episodes / truncated after 15 years) } & -- & -- & -- & -- \\ \end{array}$


Table 34: Cumulative percent ever out of union, by age of child, for children born in a consensual union $\begin{array}{ccccc}\text { GDR } & \text { Hungary } & \text { Czech R } & \text { Slovenia } & \text { Latvia } \\ (1984-89) & (1988-93) & (1992-97) & (1989-95) & (1989-95)\end{array}$

$\begin{array}{lccccc}0 & 0 & 0 & 0 & 0 & 0 \\ 1 & 6 & 7 & 9 & 3 & 16 \\ 2 & 13 & 11 & 20 & 7 & 24 \\ 3 & 19 & 17 & 27 & 8 & 36 \\ 4 & 23 & 26 & 32 & 10 & 41 \\ 6 & 28 & 42 & 32 & 12 & 51 \\ 9 & 38 & 48 & -- & 14 & 58 \\ 12 & 51 & 52 & -- & 19 & 63 \\ 15 & -- & 58 & -- & 19 & 69 \\ \text { mean age: } & -- & 5 & -- & 5 & 4 \\ \text { (at union disruption / conditional on disruption) } & & & \\ \text { 1st decile at age: } & & & & & \\ \text { 1st quartile at: } & 2 & 2 & 2 & 4 & 3 \\ \text { median at age: } & 5 & 4 & 3 & -- & 6 \\ \text { 3rd quartile at: } & 12 & 11 & -- & -- & - \\ & -- & -- & -- & -- & \\ \text { mean duration: } & & & & & \\ \text { (of all episodes / truncated after } & -- & 9 & -- & 13 & \end{array}$

(of all episodes / truncated after 15 years)

$\begin{array}{lcc}\text { age } & \begin{array}{c}\text { Lithuania } \\ (1989-95)\end{array} & \begin{array}{c}\text { Poland } \\ (1986-91)\end{array} \\ 0 & 0 & 0 \\ 1 & 15 & 0 \\ 2 & 19 & 9 \\ 3 & -- & 12 \\ 4 & -- & 12 \\ 6 & -- & 22 \\ 9 & -- & 26 \\ 12 & -- & -- \\ 15 & -- & -- \\ & & -- \\ \text { mean age: } & -- & \\ \text { (at union disruption / conditional on disruption) } \\ \text { 1st decile at age: } & & 3 \\ \text { 1st quartile at: } & 1 & 7 \\ \text { median at age: } & -- & -- \\ \text { 3rd quartile at: } & -- & -- \\ \text { mean duration: } & -- & - \\ \text { (of all episodes / truncated after 15 years) }\end{array}$


Table 35: Cumulative percent ever out of union/marriage, by age of child, for children born in a marriage

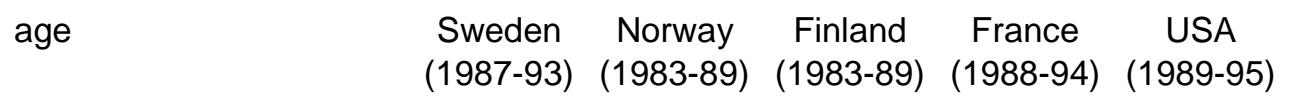

\begin{tabular}{|c|c|c|c|c|c|}
\hline 0 & 0 & 0 & 0 & 0 & 0 \\
\hline 1 & 1 & 1 & 1 & 1 & 3 \\
\hline 2 & 2 & 2 & 2 & 2 & 7 \\
\hline 3 & 5 & 4 & 4 & 3 & 10 \\
\hline 4 & 6 & 6 & 5 & 5 & 13 \\
\hline 6 & 10 & 8 & 8 & 7 & 18 \\
\hline 9 & 15 & 12 & 11 & 11 & 24 \\
\hline 12 & 21 & 16 & 14 & 14 & 29 \\
\hline 15 & 24 & 18 & 17 & 17 & 35 \\
\hline $\begin{array}{l}\text { mean age: } \\
\text { (at union disruptio }\end{array}$ & $\begin{array}{l}7 \\
\text { anal o }\end{array}$ & $\begin{array}{c}7 \\
\text { otion }\end{array}$ & 7 & 7 & 6 \\
\hline 1st decile at age: & 6 & 7 & 8 & 8 & 3 \\
\hline 1st quartile at: & -- & -- & -- & -- & 10 \\
\hline median at age: & -- & -- & -- & -- & -- \\
\hline 3rd quartile at: & -- & -- & -- & -- & -- \\
\hline mean duration: & 13 & 14 & 14 & 14 & 12 \\
\hline
\end{tabular}

(of all episodes / truncated after 15 years)

\begin{tabular}{|c|c|c|c|c|c|}
\hline age & $\begin{array}{c}\text { Austria } \\
(1990-96)\end{array}$ & $\begin{array}{c}\text { West } \\
\text { Germany } \\
(1986-92)\end{array}$ & $\begin{array}{c}\text { Flanders } \\
(1985-92)\end{array}$ & $\begin{array}{c}\text { Italy } \\
(1990-95)\end{array}$ & $\begin{array}{c}\text { Spain } \\
(1989-95)\end{array}$ \\
\hline 0 & 0 & 0 & 0 & 0 & 0 \\
\hline 1 & 1 & 1 & 1 & 0 & 1 \\
\hline 2 & 2 & 3 & 1 & 1 & 1 \\
\hline 3 & 4 & 5 & 2 & 1 & 2 \\
\hline 4 & 7 & 7 & 3 & 1 & 3 \\
\hline 6 & 11 & 9 & 6 & 2 & 4 \\
\hline 9 & 15 & 13 & 9 & 4 & 6 \\
\hline 12 & 19 & 19 & 12 & 5 & 7 \\
\hline 15 & 23 & 26 & 14 & 7 & 9 \\
\hline $\begin{array}{l}\text { mean age: } \\
\text { (at union disruptio }\end{array}$ & $\begin{array}{c}7 \\
\text { itional on di }\end{array}$ & $\begin{array}{c}8 \\
\text { sruption) }\end{array}$ & 7 & 8 & 7 \\
\hline 1st decile at age: & 6 & 7 & 10 & -- & -- \\
\hline 1st quartile at: & -- & 15 & -- & -- & -- \\
\hline median at age: & -- & -- & -- & -- & -- \\
\hline 3rd quartile at: & -- & -- & -- & -- & -- \\
\hline $\begin{array}{l}\text { mean duration: } \\
\text { (of all episodes / th }\end{array}$ & $\begin{array}{c}13 \\
\text { after } 15 \text { ye }\end{array}$ & ears) & 14 & 15 & 14 \\
\hline
\end{tabular}


Table 35: Cumulative percent ever out of union/marriage, by age of child, for children born in a marriage

\begin{tabular}{|c|c|c|c|c|c|}
\hline age & $\begin{array}{c}\text { GDR } \\
(1984-89)\end{array}$ & $\begin{array}{c}\text { Hungary } \\
(1988-93)\end{array}$ & $\begin{array}{c}\text { Czech R } \\
(1992-97)\end{array}$ & $\begin{array}{c}\text { Slovenia } \\
(1989-95)\end{array}$ & $\begin{array}{c}\text { Latvia } \\
(1989-95)\end{array}$ \\
\hline 0 & 0 & 0 & 0 & 0 & 0 \\
\hline 1 & 1 & 1 & 1 & 0 & 3 \\
\hline 2 & 3 & 2 & 3 & 0 & 7 \\
\hline 3 & 6 & 4 & 5 & 1 & 10 \\
\hline 4 & 10 & 5 & 7 & 1 & 14 \\
\hline 6 & 15 & 9 & 11 & 3 & 18 \\
\hline 9 & 21 & 12 & 15 & 5 & 24 \\
\hline 12 & 26 & 17 & 19 & 7 & 30 \\
\hline 15 & 31 & 20 & 24 & 8 & 34 \\
\hline mean age: & 7 & 7 & 7 & 8 & 6 \\
\hline \multicolumn{6}{|c|}{ (at union disruption / conditional on disruption) } \\
\hline 1st decile at age: & 4 & 7 & 5 & -- & 3 \\
\hline 1st quartile at: & 11 & -- & -- & -- & 10 \\
\hline median at age: & -- & -- & -- & -- & -- \\
\hline 3rd quartile at: & -- & -- & -- & -- & -- \\
\hline mean duration: & 12 & 13 & 13 & 14 & 12 \\
\hline
\end{tabular}

\begin{tabular}{lcc} 
age & $\begin{array}{c}\text { Lithuania } \\
(1989-95)\end{array}$ & $\begin{array}{c}\text { Poland } \\
(1986-91)\end{array}$ \\
0 & 0 & 0 \\
1 & 1 & 1 \\
2 & 3 & 2 \\
3 & 5 & 2 \\
4 & 7 & 3 \\
6 & 10 & 3 \\
9 & 15 & 5 \\
12 & 19 & 8 \\
15 & 24 & 10 \\
& & \\
mean age: & 7 & 8 \\
(at union disruption / conditional on disruption) \\
1st decile at age: & \multicolumn{2}{c}{} \\
1st quartile at: & -- & 15 \\
median at age: & -- & -- \\
3rd quartile at: & -- & -- \\
& \multicolumn{2}{c}{14} \\
mean duration: & 13 & 14 \\
(of all episodes / truncated after 15 years)
\end{tabular}


Table 36: Cumulative percent ever in union, by age of child, for children born to a lone mother

\begin{tabular}{|c|c|c|c|c|c|}
\hline age & $\begin{array}{l}\text { Sweden } \\
(1987-93)\end{array}$ & $\begin{array}{c}\text { Norway } \\
(1983-89)\end{array}$ & $\begin{array}{c}\text { Finland } \\
(1983-89)\end{array}$ & $\begin{array}{c}\text { France } \\
(1988-94)\end{array}$ & $\begin{array}{c}\text { USA } \\
(1989-95\end{array}$ \\
\hline 0 & 0 & 0 & 0 & 0 & 0 \\
\hline 1 & 19 & 29 & 25 & 8 & 18 \\
\hline 2 & 27 & 39 & 30 & 12 & 29 \\
\hline 3 & 29 & 54 & 39 & 13 & 37 \\
\hline 4 & 41 & 64 & 39 & 20 & 44 \\
\hline 6 & 43 & -- & 53 & 22 & 55 \\
\hline 9 & 52 & -- & 64 & 38 & 65 \\
\hline 12 & -- & -- & -- & 38 & 71 \\
\hline 15 & -- & -- & -- & 39 & 78 \\
\hline $\begin{array}{l}\text { mean age: } \\
\text { (at entry to union }\end{array}$ & onal on unio & n formation & -- & 5 & 5 \\
\hline 1st decile at age: & 1 & 1 & 1 & 2 & 1 \\
\hline 1st quartile at: & 2 & 1 & 1 & 8 & 2 \\
\hline median at age: & 9 & 3 & 6 & -- & 5 \\
\hline 3rd quartile at: & -- & 5 & -- & -- & 14 \\
\hline $\begin{array}{l}\text { mean duration: } \\
\text { (of all episodes / t }\end{array}$ & $\stackrel{--}{\text { fter } 15 \text { ye }}$ & rs) & -- & 11 & 7 \\
\hline
\end{tabular}

$\begin{array}{lccccc}\text { age } & \begin{array}{c}\text { West } \\ \text { Austria } \\ (1990-96)\end{array} & \begin{array}{c}\text { Germany } \\ (1986-92)\end{array} & \begin{array}{c}\text { Flanders } \\ (1985-92)\end{array} & \begin{array}{c}\text { Italy } \\ (1990-95)\end{array} & \begin{array}{c}\text { Spain } \\ (1989-95)\end{array} \\ & & & & & \\ 0 & 0 & 0 & 0 & 0 & 0 \\ 1 & 19 & 12 & 5 & 48 & 46 \\ 2 & 32 & 27 & 20 & -- & -- \\ 3 & 44 & 32 & -- & -- & -- \\ 4 & 51 & 35 & -- & -- & -- \\ 6 & 60 & -- & -- & -- & -- \\ 9 & 73 & -- & -- & -- & - \\ 12 & 80 & -- & -- & -- & - \\ 15 & 85 & -- & -- & -- & -- \\ \text { mean age: } & 4 & -- & -- & -- & -- \\ \text { (at entry to union / conditional on union formation) } & & & & \\ \text { 1st decile at age: } & 1 & 1 & 2 & 1 & 1 \\ \text { 1st quartile at: } & 2 & 2 & -- & 1 & 1 \\ \text { median at age: } & 4 & -- & -- & 2 & 2 \\ \text { 3rd quartile at: } & 11 & -- & -- & -- & -- \\ & \\ \text { mean duration: } & 6 & -- & -- & -- & -- \\ \text { (of all episodes / truncated after 15 years) } & & & & \end{array}$


Table 36: Cumulative percent ever in union, by age of child, for children born to a lone mother

\begin{tabular}{|c|c|c|c|c|c|}
\hline age & $\begin{array}{c}\text { GDR } \\
(1984-89)\end{array}$ & $\begin{array}{l}\text { Hungary } \\
(1988-93)\end{array}$ & $\begin{array}{c}\text { Czech R } \\
(1992-97)\end{array}$ & $\begin{array}{c}\text { Slovenia } \\
(1989-95)\end{array}$ & $\begin{array}{c}\text { Latvia } \\
\text { (1989-95 }\end{array}$ \\
\hline 0 & 0 & 0 & 0 & 0 & 0 \\
\hline 1 & 37 & 23 & 17 & 42 & 25 \\
\hline 2 & 55 & 37 & 35 & 59 & 43 \\
\hline 3 & 63 & 52 & -- & 66 & 52 \\
\hline 4 & 72 & 62 & -- & 78 & 58 \\
\hline 6 & 80 & -- & -- & 82 & 63 \\
\hline 9 & 87 & -- & -- & 85 & 69 \\
\hline 12 & -- & -- & -- & -- & 72 \\
\hline 15 & -- & -- & -- & -- & -- \\
\hline \multicolumn{6}{|c|}{ (at entry to union / conditional on union formation) } \\
\hline 1st decile at age: & 1 & 1 & 1 & 1 & 1 \\
\hline 1st quartile at: & 1 & 2 & 2 & 1 & 1 \\
\hline median at age: & 2 & 3 & -- & 2 & 3 \\
\hline 3rd quartile at: & 5 & -- & -- & 4 & -- \\
\hline $\begin{array}{l}\text { mean duration: } \\
\text { (of all episodes / } t\end{array}$ & $\begin{array}{c}-- \\
\text { after } 15 y\end{array}$ & rs) & -- & -- & -- \\
\hline
\end{tabular}

\begin{tabular}{|c|c|c|}
\hline age & $\begin{array}{l}\text { Lithuania } \\
(1989-95)\end{array}$ & $\begin{array}{l}\text { Poland } \\
(1986-91)\end{array}$ \\
\hline 0 & 0 & 0 \\
\hline 1 & 21 & 17 \\
\hline 2 & 26 & 21 \\
\hline 3 & 32 & 25 \\
\hline 4 & 36 & 28 \\
\hline 6 & 45 & 30 \\
\hline 9 & -- & 32 \\
\hline 12 & -- & 35 \\
\hline 15 & -- & 36 \\
\hline mean age: & -- & 3 \\
\hline \multicolumn{3}{|c|}{ (at entry to union / conditional on union formation) } \\
\hline 1st decile at age: & 1 & 1 \\
\hline 1st quartile at: & 2 & 3 \\
\hline median at age: & 7 & -- \\
\hline 3rd quartile at: & -- & -- \\
\hline mean duration: & -- & 11 \\
\hline
\end{tabular}


Table 37: Cumulative percent ever in marriage, by age of child, for children born to a lone mother $\begin{array}{ccccc}\text { Sweden } & \text { Norway } & \text { Finland } & \text { France } & \text { USA } \\ (1987-93) & (1983-89) & (1983-89) & (1988-94) & (1989-95)\end{array}$

\begin{tabular}{|c|c|c|c|c|c|}
\hline 0 & 0 & 0 & 0 & 0 & 0 \\
\hline 1 & 1 & 6 & 5 & 2 & 6 \\
\hline 2 & 3 & 13 & 5 & 3 & 11 \\
\hline 3 & 7 & 17 & 14 & 3 & 17 \\
\hline 4 & 11 & 23 & 16 & 3 & 23 \\
\hline 6 & 15 & 33 & 19 & 8 & 32 \\
\hline 9 & 25 & 43 & 29 & 19 & 42 \\
\hline 12 & 32 & 50 & 34 & 19 & 50 \\
\hline 15 & 35 & -- & -- & 19 & 58 \\
\hline \multicolumn{6}{|c|}{ (at entry to marriage / conditional on marriage formation) } \\
\hline 1st decile at age: & 4 & 2 & 3 & 7 & 2 \\
\hline 1st quartile at: & 9 & 5 & 8 & -- & 5 \\
\hline median at age: & -- & 12 & -- & -- & 12 \\
\hline 3rd quartile at: & -- & -- & -- & -- & -- \\
\hline $\begin{array}{l}\text { mean duration: } \\
\text { (of all episodes / t }\end{array}$ & $\begin{array}{l}12 \\
\text { er } 1\end{array}$ & 10 & -- & 13 & 10 \\
\hline
\end{tabular}

\begin{tabular}{|c|c|c|c|c|c|}
\hline age & $\begin{array}{c}\text { Austria } \\
(1990-96)\end{array}$ & $\begin{array}{c}\text { West } \\
\text { Germany } \\
(1986-92)\end{array}$ & $\begin{array}{l}\text { Flanders } \\
(1985-92)\end{array}$ & $\begin{array}{c}\text { Italy } \\
(1990-95)\end{array}$ & $\begin{array}{c}\text { Spain } \\
(1989-95)\end{array}$ \\
\hline 0 & 0 & 0 & 0 & 0 & 0 \\
\hline 1 & 5 & 9 & 5 & 28 & 37 \\
\hline 2 & 14 & 14 & 10 & -- & 44 \\
\hline 3 & 19 & 17 & -- & -- & 50 \\
\hline 4 & 26 & 24 & -- & -- & 56 \\
\hline 6 & 35 & 39 & -- & -- & -- \\
\hline 9 & 48 & 45 & -- & -- & -- \\
\hline 12 & 62 & -- & -- & -- & -- \\
\hline 15 & 69 & -- & -- & -- & -- \\
\hline \multicolumn{6}{|c|}{ (at entry to marriage / conditional on marriage formation) } \\
\hline 1st decile at age: & 2 & 2 & 2 & 1 & 1 \\
\hline 1st quartile at: & 4 & 5 & -- & 1 & 1 \\
\hline median at age: & 10 & -- & -- & -- & 3 \\
\hline 3rd quartile at: & -- & -- & -- & -- & -- \\
\hline $\begin{array}{l}\text { mean duration: } \\
\text { (of all episodes / } t\end{array}$ & $\begin{array}{c}9 \\
\text { after } 15 y\end{array}$ & rs) & -- & -- & -- \\
\hline
\end{tabular}


Table 37: Cumulative percent ever in marriage, by age of child, for children born to a lone mother

\begin{tabular}{|c|c|c|c|c|c|}
\hline age & $\begin{array}{c}\text { GDR } \\
(1984-89)\end{array}$ & $\begin{array}{l}\text { Hungary } \\
(1988-93)\end{array}$ & $\begin{array}{c}\text { Czech R } \\
(1992-97)\end{array}$ & $\begin{array}{c}\text { Slovenia } \\
(1989-95)\end{array}$ & $\begin{array}{c}\text { Latvia } \\
(1989-95\end{array}$ \\
\hline 0 & 0 & 0 & 0 & 0 & 0 \\
\hline 1 & 22 & 11 & 4 & 18 & 15 \\
\hline 2 & 34 & 18 & 13 & 28 & 27 \\
\hline 3 & 42 & 30 & 29 & 32 & 30 \\
\hline 4 & 50 & 39 & 33 & 39 & 34 \\
\hline 6 & 60 & -- & -- & 47 & 34 \\
\hline 9 & 67 & -- & -- & 55 & 40 \\
\hline 12 & 72 & -- & -- & 59 & 43 \\
\hline 15 & -- & -- & -- & -- & -- \\
\hline \multicolumn{5}{|c|}{ (at entry to marriage / conditional on marriage formation) } & -- \\
\hline 1st decile at age: & 1 & 1 & 2 & 1 & 1 \\
\hline 1st quartile at: & 2 & 3 & 3 & 2 & 2 \\
\hline median at age: & 4 & -- & -- & 8 & -- \\
\hline 3rd quartile at: & 14 & -- & -- & -- & -- \\
\hline $\begin{array}{l}\text { mean duration: } \\
\text { (of all episodes / t }\end{array}$ & after $15 \mathrm{yc}$ & ars) & -- & -- & -- \\
\hline
\end{tabular}

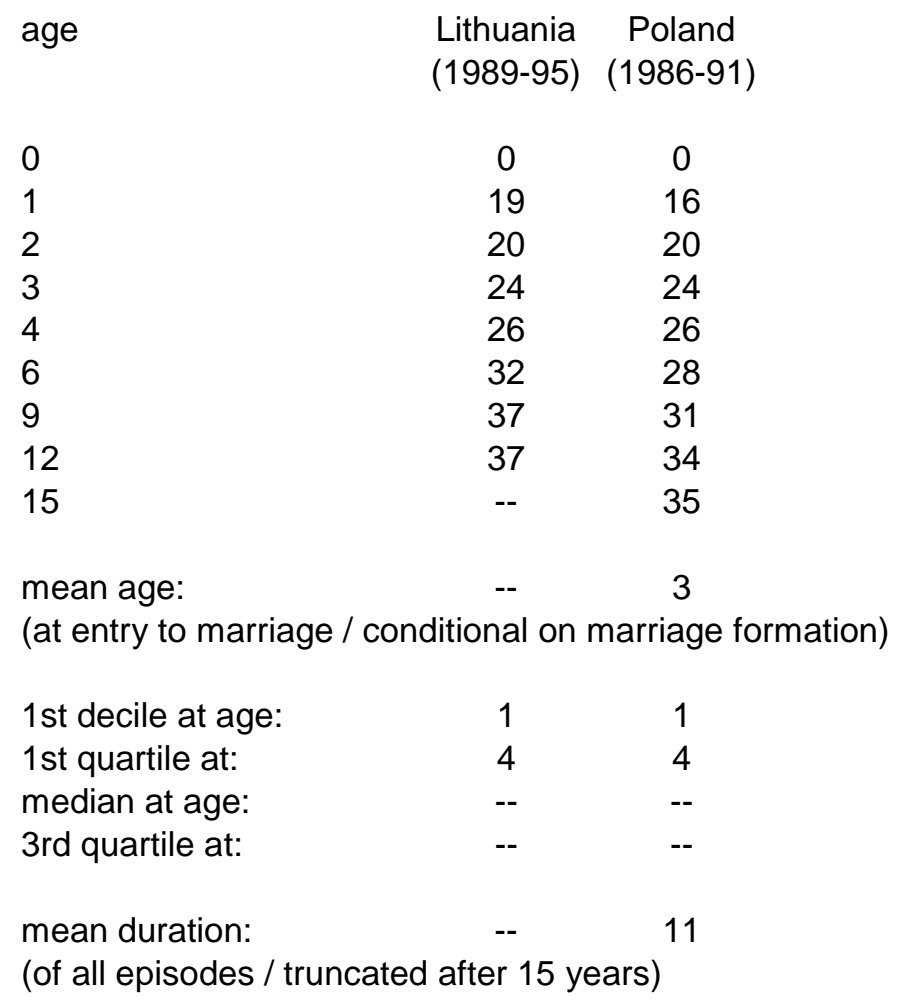


Table 38: Cumulative percent ever in marriage, by age of child, for children born in a consensual union

\begin{tabular}{|c|c|c|c|c|c|}
\hline age & $\begin{array}{c}\text { Sweden } \\
(1987-93)\end{array}$ & $\begin{array}{c}\text { Norway } \\
(1983-89)\end{array}$ & $\begin{array}{l}\text { Finland } \\
(1983-89)\end{array}$ & $\begin{array}{c}\text { France } \\
(1988-94)\end{array}$ & $\begin{array}{c}\text { USA } \\
(1989-95\end{array}$ \\
\hline 0 & 0 & 0 & 0 & 0 & 0 \\
\hline 1 & 17 & 16 & 22 & 9 & 15 \\
\hline 2 & 27 & 28 & 27 & 17 & 27 \\
\hline 3 & 38 & 36 & 31 & 19 & 35 \\
\hline 4 & 45 & 40 & 32 & 24 & 41 \\
\hline 6 & 56 & 45 & 41 & 30 & 52 \\
\hline 9 & 68 & 51 & 51 & 32 & 62 \\
\hline 12 & 76 & -- & 57 & 33 & 66 \\
\hline 15 & 81 & -- & -- & 33 & 69 \\
\hline \multicolumn{6}{|c|}{ (at entry to marriage / conditional on marriage formation) } \\
\hline 1st decile at age: & 1 & 1 & 1 & 2 & 1 \\
\hline 1st quartile at: & 2 & 2 & 2 & 5 & 2 \\
\hline median at age: & 5 & 9 & 8 & -- & 6 \\
\hline 3rd quartile at: & 12 & -- & -- & -- & -- \\
\hline $\begin{array}{l}\text { mean duration: } \\
\text { (of all episodes / t }\end{array}$ & $\begin{array}{c}7 \\
\text { after } 15 y\end{array}$ & rs) & -- & 11 & 7 \\
\hline
\end{tabular}

\begin{tabular}{|c|c|c|c|c|c|}
\hline age & $\begin{array}{c}\text { Austria } \\
(1990-96)\end{array}$ & $\begin{array}{c}\text { West } \\
\text { Germany } \\
(1986-92)\end{array}$ & $\begin{array}{l}\text { Flanders } \\
(1985-92)\end{array}$ & $\begin{array}{c}\text { Italy } \\
(1990-95)\end{array}$ & $\begin{array}{c}\text { Spain } \\
(1989-95)\end{array}$ \\
\hline 0 & 0 & 0 & 0 & 0 & 0 \\
\hline 1 & 12 & 17 & 5 & 17 & 5 \\
\hline 2 & 26 & 26 & 16 & 23 & 5 \\
\hline 3 & 35 & 34 & 24 & 25 & 5 \\
\hline 4 & 43 & 35 & 36 & 43 & 5 \\
\hline 6 & 54 & 40 & -- & 54 & 16 \\
\hline 9 & 68 & 45 & -- & -- & -- \\
\hline 12 & 71 & 49 & -- & -- & -- \\
\hline 15 & -- & -- & -- & -- & -- \\
\hline $\begin{array}{l}\text { mean age: } \\
\text { (at entry to marria }\end{array}$ & $\begin{array}{l}-- \\
\text { ditional on }\end{array}$ & $\stackrel{--}{\text { marriage fo }}$ & $\begin{array}{c}-- \\
\text { rmation) }\end{array}$ & -- & -- \\
\hline 1st decile at age: & 1 & 1 & 2 & 1 & 6 \\
\hline 1st quartile at: & 2 & 2 & 4 & 3 & 8 \\
\hline median at age: & 6 & 13 & 5 & 5 & -- \\
\hline 3rd quartile at: & -- & -- & -- & -- & -- \\
\hline $\begin{array}{l}\text { mean duration: } \\
\text { (of all episodes / t }\end{array}$ & $\begin{array}{l}-- \\
\text { fter } 15\end{array}$ & rs) & -- & -- & -- \\
\hline
\end{tabular}


Table 38: Cumulative percent ever in marriage, by age of child, for children born in a consensual union

\begin{tabular}{|c|c|c|c|c|c|}
\hline age & $\begin{array}{c}\text { GDR } \\
(1984-89)\end{array}$ & $\begin{array}{l}\text { Hungary } \\
(1988-93)\end{array}$ & $\begin{array}{c}\text { Czech R } \\
(1992-97)\end{array}$ & $\begin{array}{c}\text { Slovenia } \\
(1989-95)\end{array}$ & $\begin{array}{c}\text { Latvia } \\
(1989-95)\end{array}$ \\
\hline 0 & 0 & 0 & 0 & 0 & 0 \\
\hline 1 & 26 & 11 & 19 & 17 & 14 \\
\hline 2 & 39 & 18 & 26 & 26 & 18 \\
\hline 3 & 47 & 22 & 31 & 30 & 19 \\
\hline 4 & 55 & 25 & 38 & 35 & 22 \\
\hline 6 & 63 & 36 & -- & 42 & 28 \\
\hline 9 & 69 & 49 & -- & 51 & 32 \\
\hline 12 & -- & -- & -- & 54 & 32 \\
\hline 15 & -- & -- & -- & 56 & 32 \\
\hline mean age: & -- & -- & -- & 4 & 3 \\
\hline \multicolumn{6}{|c|}{ (at entry to marriage / conditional on marriage formation) } \\
\hline 1st decile at age: & 1 & 1 & 1 & 1 & 1 \\
\hline 1st quartile at: & 1 & 4 & 2 & 2 & 5 \\
\hline median at age: & 4 & -- & -- & 9 & -- \\
\hline 3rd quartile at: & -- & -- & -- & -- & -- \\
\hline $\begin{array}{l}\text { mean duration: } \\
\text { (of all episodes / }\end{array}$ & after $15 \mathrm{y \epsilon}$ & ars) & -- & 9 & 11 \\
\hline
\end{tabular}

$\begin{array}{lcc}\text { age } & \begin{array}{c}\text { Lithuania } \\ (1989-95)\end{array} & \begin{array}{c}\text { Poland } \\ (1986-91)\end{array} \\ 0 & 0 & 0 \\ 1 & 14 & 3 \\ 2 & 23 & 11 \\ 3 & -- & 15 \\ 4 & -- & -- \\ 6 & -- & -- \\ 9 & -- & -- \\ 12 & -- & -- \\ 15 & -- & -- \\ & & \\ \text { mean age: } & -- & -- \\ \text { (at entry to marriage / conditional on marriage formation) } \\ \text { 1st decile at age: } & 1 & \\ \text { 1st quartile at: } & -- & -- \\ \text { median at age: } & -- & -- \\ \text { 3rd quartile at: } & -- & -- \\ & & \\ \text { mean duration: } & -- & - \\ \text { (of all episodes / truncated after 15 years) }\end{array}$


Table 39: Cumulative percent ever in marriage, by age of child, for children born to a non-married mother

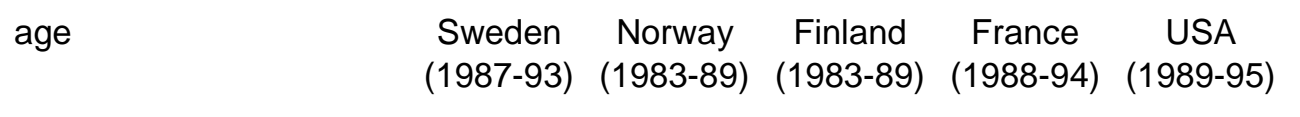

\begin{tabular}{|c|c|c|c|c|c|}
\hline 0 & 0 & 0 & 0 & 0 & 0 \\
\hline 1 & 15 & 13 & 19 & 7 & 10 \\
\hline 2 & 26 & 24 & 22 & 12 & 17 \\
\hline 3 & 35 & 31 & 28 & 14 & 24 \\
\hline 4 & 43 & 36 & 29 & 18 & 30 \\
\hline 6 & 53 & 42 & 36 & 24 & 40 \\
\hline 9 & 65 & 50 & 46 & 29 & 50 \\
\hline 12 & 73 & 55 & 52 & 29 & 56 \\
\hline 15 & 77 & 58 & 56 & 29 & 62 \\
\hline mean age: & 5 & 4 & 4 & 3 & 5 \\
\hline 1st decile at age: & 1 & 1 & 1 & 2 & 1 \\
\hline 1st quartile at: & 2 & 3 & 3 & 7 & 4 \\
\hline median at age: & 6 & 9 & 11 & -- & 9 \\
\hline 3rd quartile at: & 14 & -- & -- & -- & -- \\
\hline $\begin{array}{l}\text { mean duration: } \\
\text { (of all episodes / th }\end{array}$ & 7 & 9 & 9 & 12 & 9 \\
\hline
\end{tabular}

\begin{tabular}{|c|c|c|c|c|c|}
\hline age & $\begin{array}{c}\text { Austria } \\
(1990-96)\end{array}$ & $\begin{array}{c}\text { West } \\
\text { Germany } \\
(1986-92)\end{array}$ & $\begin{array}{c}\text { Flanders } \\
(1985-92)\end{array}$ & $\begin{array}{c}\text { Italy } \\
(1990-95)\end{array}$ & $\begin{array}{c}\text { Spain } \\
(1989-95)\end{array}$ \\
\hline 0 & 0 & 0 & 0 & 0 & 0 \\
\hline 1 & 10 & 14 & 5 & 20 & 16 \\
\hline 2 & 21 & 22 & 14 & 26 & 19 \\
\hline 3 & 29 & 27 & 23 & 29 & 22 \\
\hline 4 & 37 & 31 & 31 & 48 & 25 \\
\hline 6 & 47 & 39 & 42 & 56 & 32 \\
\hline 9 & 60 & 45 & -- & 68 & 41 \\
\hline 12 & 68 & 49 & -- & -- & -- \\
\hline 15 & 73 & 55 & -- & -- & -- \\
\hline $\begin{array}{l}\text { mean age: } \\
\text { (at entry to marria }\end{array}$ & $\begin{array}{c}5 \\
\text { ditional on } r\end{array}$ & $\begin{array}{c}5 \\
\text { narriage for }\end{array}$ & mation) & -- & -- \\
\hline 1st decile at age: & 1 & 1 & 2 & 1 & 1 \\
\hline 1st quartile at: & 3 & 3 & 4 & 2 & 4 \\
\hline median at age: & 7 & 13 & -- & 5 & -- \\
\hline 3rd quartile at: & -- & -- & -- & -- & -- \\
\hline $\begin{array}{l}\text { mean duration: } \\
\text { (of all episodes / tr }\end{array}$ & $\begin{array}{c}8 \\
\text { after } 15 \text { ye }\end{array}$ & 9 & -- & -- & -- \\
\hline
\end{tabular}


Table 39: Cumulative percent ever in marriage, by age of child, for children born to a non-married mother

\begin{tabular}{|c|c|c|c|c|c|}
\hline age & $\begin{array}{c}\text { GDR } \\
(1984-89)\end{array}$ & $\begin{array}{c}\text { Hungary } \\
(1988-93)\end{array}$ & $\begin{array}{c}\text { Czech R } \\
(1992-97)\end{array}$ & $\begin{array}{c}\text { Slovenia } \\
(1989-95)\end{array}$ & $\begin{array}{c}\text { Latvia } \\
\text { (1989-95 }\end{array}$ \\
\hline 0 & 0 & 0 & 0 & 0 & 0 \\
\hline 1 & 24 & 11 & 13 & 17 & 14 \\
\hline 2 & 37 & 18 & 21 & 27 & 22 \\
\hline 3 & 44 & 25 & 30 & 31 & 24 \\
\hline 4 & 52 & 30 & 36 & 36 & 28 \\
\hline 6 & 61 & 45 & 43 & 43 & 31 \\
\hline 9 & 68 & 57 & -- & 52 & 36 \\
\hline 12 & 72 & -- & -- & 56 & 38 \\
\hline 15 & 76 & -- & -- & 58 & 40 \\
\hline mean age: & 4 & -- & -- & 4 & 4 \\
\hline \multicolumn{6}{|c|}{ (at entry to marriage / conditional on marriage formation) } \\
\hline 1st decile at age: & 1 & 1 & 1 & 1 & 1 \\
\hline 1st quartile at: & 2 & 3 & 3 & 2 & 4 \\
\hline median at age: & 4 & 7 & 8 & 8 & -- \\
\hline 3rd quartile at: & 15 & -- & -- & -- & -- \\
\hline $\begin{array}{l}\text { mean duration: } \\
\text { (of all episodes / tr }\end{array}$ & $\begin{array}{c}6 \\
\text { after } 15 \text { J }\end{array}$ & -- & -- & 8 & 10 \\
\hline
\end{tabular}

\begin{tabular}{|c|c|c|}
\hline age & $\begin{array}{l}\text { Lithuania } \\
(1989-95)\end{array}$ & $\begin{array}{l}\text { Poland } \\
(1986-91)\end{array}$ \\
\hline 0 & 0 & 0 \\
\hline 1 & 17 & 13 \\
\hline 2 & 21 & 18 \\
\hline 3 & 28 & 22 \\
\hline 4 & 33 & 24 \\
\hline 6 & 41 & 27 \\
\hline 9 & 46 & 29 \\
\hline 12 & 50 & 32 \\
\hline 15 & -- & 33 \\
\hline \multicolumn{3}{|c|}{ (at entry to marriage / conditional on marriage formation) } \\
\hline 1st decile at age: & 1 & 1 \\
\hline 1st quartile at: & 3 & 5 \\
\hline median at age: & 10 & -- \\
\hline 3rd quartile at: & -- & -- \\
\hline $\begin{array}{l}\text { mean duration: } \\
\text { (of all episodes / tr }\end{array}$ & $\begin{array}{c}9 \\
\text { after } 15 \text { ye }\end{array}$ & ears) \\
\hline
\end{tabular}


Table 40: Cumulative percent in marriage, by age of child, for children born in a consensual union, competing-risks life-table method with family dissolution as competing event

$\begin{array}{lccccc}\text { age } & \begin{array}{c}\text { Sweden } \\ (1987-93)\end{array} & \begin{array}{c}\text { Norway } \\ (1983-89)\end{array} & \begin{array}{c}\text { Finland } \\ (1983-89)\end{array} & \begin{array}{c}\text { France } \\ (1988-94)\end{array} & \begin{array}{c}\text { USA } \\ (1989-95)\end{array} \\ 0 & & & & & \\ 1 & 0 & 0 & 0 & 0 & 0 \\ 2 & 16 & 15 & 22 & 9 & 15 \\ 3 & 27 & 28 & 27 & 17 & 25 \\ 4 & 37 & 36 & 31 & 19 & 31 \\ 6 & 44 & 40 & 31 & 24 & 34 \\ 9 & 54 & 44 & 38 & 30 & 40 \\ 12 & 62 & -- & 44 & 32 & 45 \\ 15 & 67 & -- & 46 & 32 & 47 \\ & 70 & -- & -- & 32 & -- \\ \text { mean age: } & & & & & \\ \text { (at entry to marriage / conditional on marriage formation) } & & \\ & & & & & \\ \text { 1st decile at age: } & 1 & 1 & 1 & 2 & 1 \\ \text { 1st quartile at: } & 2 & 2 & 2 & 5 & 2 \\ \text { median at age: } & 6 & -- & -- & -- & -- \\ \text { 3rd quartile at: } & -- & -- & -- & -- & --\end{array}$

$\begin{array}{lccccc}\text { age } & \begin{array}{c}\text { West } \\ \text { Austria } \\ (1990-96)\end{array} & \begin{array}{c}\text { Germany } \\ (1986-92)\end{array} & \begin{array}{c}\text { Flanders } \\ (1985-92)\end{array} & \begin{array}{c}\text { Italy } \\ (1990-95)\end{array} & \begin{array}{c}\text { Spain } \\ (1989-95)\end{array} \\ 0 & & & & & \\ 1 & 0 & 0 & 0 & 0 & 0 \\ 2 & 12 & 17 & 5 & 17 & 5 \\ 3 & 25 & 26 & 16 & 23 & 5 \\ 4 & 33 & 33 & 21 & 25 & 5 \\ 6 & 41 & 35 & 33 & 42 & 5 \\ 9 & 51 & 38 & -- & -- & 11 \\ 12 & 61 & 43 & -- & -- & -- \\ 15 & -- & -- & -- & -- & - \\ & -- & -- & -- & -- & -- \\ \text { mean age: } & -- & -- & -- & -- & -- \\ \text { (at entry to marriage / conditional on marriage formation) } & & \\ \text { 1st decile at age: } & & & & & \\ \text { 1st quartile at: } & 1 & 1 & 2 & 1 & 6 \\ \text { median at age: } & 2 & 2 & 4 & 3 & -- \\ \text { 3rd quartile at: } & 6 & -- & -- & 5 & -- \\ & -- & -- & -- & -- & -\end{array}$


Table 40: Cumulative percent in marriage, by age of child, for children born in a consensual union, competing-risks life-table method with family dissolution as competing event

$\begin{array}{lccccc}\text { age } & \begin{array}{c}\text { GDR } \\ (1984-89)\end{array} & \begin{array}{c}\text { Hungary } \\ (1988-93)\end{array} & \begin{array}{c}\text { Czech R } \\ (1992-97)\end{array} & \begin{array}{c}\text { Slovenia } \\ (1989-95)\end{array} & \begin{array}{c}\text { Latvia } \\ (1989-95)\end{array} \\ 0 & & & & & \\ 1 & 0 & 0 & 0 & 0 & 0 \\ 2 & 25 & 11 & 19 & 17 & 13 \\ 3 & 38 & 17 & 23 & 26 & 16 \\ 4 & 45 & 21 & 26 & 30 & 18 \\ 6 & 50 & 24 & 32 & 35 & 19 \\ 9 & 56 & 32 & -- & 41 & 20 \\ 12 & -- & -- & -- & 49 & 22 \\ 15 & -- & -- & -- & -- & -- \\ & -- & -- & -- & -- & - \\ \text { mean age: } & -- & -- & -- & -- & -- \\ \text { (at entry to marriage / conditional on marriage formation) } & & \\ \text { 1st decile at age: } & & & & & \\ \text { 1st quartile at: } & 1 & 1 & 1 & 1 & 1 \\ \text { median at age: } & 1 & 5 & 3 & 2 & -- \\ \text { 3rd quartile at: } & 4 & -- & -- & 10 & -- \\ & -- & -- & -- & -- & --\end{array}$

$\begin{array}{ccc}\text { age } & \text { Lithuania } & \text { Poland } \\ & (1989-95) & (1986-91)\end{array}$

$\begin{array}{lcc}0 & 0 & 0 \\ 1 & 14 & 3 \\ 2 & -- & 12 \\ 3 & -- & 15 \\ 4 & -- & -- \\ 6 & -- & -- \\ 9 & -- & -- \\ 12 & -- & -- \\ 15 & -- & --\end{array}$

mean age:

(at entry to marriage / conditional on marriage formation)

1st decile at age: $\quad 1 \quad 2$

1st quartile at: - -- $\quad$--

median at age: $\quad--\quad--$

3rd quartile at: $\quad$-- $\quad$-- 
Table 41: Cumulative percent out of union, by age of child, for children born in a consensual union, competing-risks life-table method with marriage formation of parents as competing event

$\begin{array}{lccccc}\text { age } & \begin{array}{c}\text { Sweden } \\ (1987-93)\end{array} & \begin{array}{c}\text { Norway } \\ (1983-89)\end{array} & \begin{array}{c}\text { Finland } \\ (1983-89)\end{array} & \begin{array}{c}\text { France } \\ (1988-94)\end{array} & \begin{array}{c}\text { USA } \\ (1989-95)\end{array} \\ 0 & & & & & \\ 1 & 0 & 0 & 0 & 0 & 0 \\ 2 & 4 & 8 & 4 & 5 & 18 \\ 3 & 8 & 13 & 10 & 10 & 27 \\ 4 & 11 & 17 & 15 & 15 & 34 \\ 6 & 14 & 18 & 18 & 18 & 38 \\ 9 & 19 & 20 & 19 & 24 & 42 \\ 12 & 20 & -- & 26 & 30 & 46 \\ 15 & 21 & -- & 26 & 40 & 48 \\ & 22 & -- & -- & 45 & -- \\ \text { mean age: } & & & & & \\ \text { (at union disruption / conditional on disruption) } & -- & 6 & 3 \\ & & & & & \\ \text { 1st decile at age: } & 3 & 2 & 2 & 2 & 1 \\ \text { 1st quartile at: } & -- & 8 & 9 & 7 & 2 \\ \text { median at age: } & -- & -- & -- & -- & -- \\ \text { 3rd quartile at: } & -- & -- & -- & -- & --\end{array}$

\begin{tabular}{lccccc} 
age & $\begin{array}{c}\text { West } \\
\text { Austria } \\
(1990-96)\end{array}$ & $\begin{array}{c}\text { Germany } \\
(1986-92)\end{array}$ & $\begin{array}{c}\text { Flanders } \\
(1985-92)\end{array}$ & $\begin{array}{c}\text { Italy } \\
(1990-95)\end{array}$ & $\begin{array}{c}\text { Spain } \\
(1989-95)\end{array}$ \\
0 & & & & & \\
1 & 0 & 0 & 0 & 0 & 0 \\
2 & 6 & 4 & 4 & 0 & 9 \\
3 & 8 & 11 & 10 & 2 & 18 \\
4 & 11 & 14 & 15 & 2 & 24 \\
6 & 17 & 16 & 24 & 2 & 27 \\
9 & 22 & 18 & -- & -- & 33 \\
12 & 22 & 24 & -- & -- & -- \\
15 & -- & -- & -- & -- & - \\
& -- & -- & -- & -- & - \\
mean age: & - & & & & \\
(at union disruption / conditional on disruption) & -- & -- & - \\
\multicolumn{1}{l|}{ 1st decile at age: } & & & & & \\
1st quartile at: & 3 & 2 & 2 & -- & 2 \\
median at age: & -- & 10 & -- & -- & 4 \\
3rd quartile at: & -- & -- & -- & -- & -- \\
& -- & -- & -- & -- & -
\end{tabular}


Table 41: Cumulative percent out of union, by age of child, for children born in a consensual union, competing-risks life-table method with marriage formation of parents as competing event
age
GDR Hungary Czech R Slovenia Latvia
(1984-89) (1988-93) (1992-97) (1989-95) $\quad$ (1989-95)

$\begin{array}{lccccc}0 & 0 & 0 & 0 & 0 & 0 \\ 1 & 6 & 7 & 7 & 3 & 14 \\ 2 & 12 & 11 & 12 & 7 & 20 \\ 3 & 17 & 16 & 12 & 7 & 33 \\ 4 & 18 & 24 & 18 & 9 & 38 \\ 6 & 21 & 39 & -- & 10 & 48 \\ 9 & -- & -- & -- & 11 & 55 \\ 12 & -- & -- & -- & -- & -- \\ 15 & -- & -- & -- & -- & -- \\ \text { mean age: } & & - & -- & -- & -- \\ \text { (at union disruption / conditional on disruption) } & & & \\ & - & & & & \\ \text { 1st decile at age: } & - & 2 & 2 & 6 & 1 \\ \text { 1st quartile at: } & -- & 5 & -- & -- & 3 \\ \text { median at age: } & -- & -- & -- & -- & 7 \\ \text { 3rd quartile at: } & -- & -- & -- & -- & -\end{array}$

age

Lithuania Poland

(1989-95) (1986-91)

\begin{tabular}{lcc}
0 & 0 & 0 \\
1 & 14 & 0 \\
2 & -- & 6 \\
3 & -- & 9 \\
4 & -- & -- \\
6 & -- & -- \\
9 & -- \\
12 & -- & -- \\
15 & -- & -- \\
\hline & -- & --
\end{tabular}

mean age:

(at union disruption / conditional on disruption)

1st decile at age:

1st quartile at:

median at age:

3rd quartile at: 
=> Table 42: Cumulative percent ever out of consensual-union status, by age of child, for children born in a consensual union

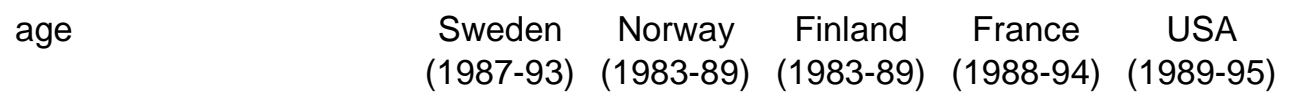

$\begin{array}{lccccc}0 & 0 & 0 & 0 & 0 & 0 \\ 1 & 20 & 23 & 26 & 14 & 33 \\ 2 & 35 & 41 & 37 & 27 & 53 \\ 3 & 48 & 54 & 46 & 34 & 65 \\ 4 & 58 & 58 & 49 & 42 & 72 \\ 6 & 73 & 64 & 57 & 54 & 82 \\ 9 & 82 & -- & 70 & 62 & 90 \\ 12 & 88 & -- & 72 & 72 & 95 \\ 15 & 92 & -- & -- & 77 & -- \\ \text { mean age: } & 4 & -- & -- & 5 & 3 \\ \text { (at exit / conditional on exit from consensual-union status) } & & \\ \text { 1st decile at age: } & 1 & 1 & 1 & 1 & 1 \\ \text { 1st quartile at: } & 2 & 2 & 1 & 2 & 1 \\ \text { median at age: } & 4 & 3 & 5 & 5 & 2 \\ \text { 3rd quartile at: } & 7 & -- & -- & 14 & 5 \\ & & & -- & 7 & 3\end{array}$

\begin{tabular}{ccccc}
\multicolumn{5}{c}{ West } \\
Austria & Germany & Flanders & Italy & Spain \\
$(1990-96)$ & $(1986-92)$ & $(1985-92)$ & $(1990-95)$ & $(1989-95)$
\end{tabular}

$\begin{array}{lccccc}0 & 0 & 0 & 0 & 0 & 0 \\ 1 & 18 & 21 & 9 & 17 & 14 \\ 2 & 33 & 38 & 26 & 25 & 23 \\ 3 & 44 & 47 & 36 & 27 & 30 \\ 4 & 58 & 51 & 57 & 44 & 32 \\ 6 & 73 & 56 & -- & -- & 45 \\ 9 & 83 & 67 & -- & -- & -- \\ 12 & -- & -- & -- & -- & -- \\ 15 & -- & -- & -- & -- & --\end{array}$

mean age:

(at exit / conditional on exit from consensual-union status)

1st decile at age:

1st quartile at:

median at age:

3rd quartile at:

$\begin{array}{lllll}1 & 1 & 2 & 1 & 1 \\ 2 & 2 & 2 & 2 & 3 \\ 4 & 4 & 4 & 5 & -- \\ 7 & -- & -- & -- & --\end{array}$

mean duration:

(of all episodes / truncated after 15 years) 
=> Table 42: Cumulative percent ever out of consensual-union status, by age of child, for children born in a consensual union
age
GDR Hungary Czech R Slovenia Latvia
$\begin{array}{lllll}(1984-89) & (1988-93) & (1992-97) & (1989-95) & (1989-95)\end{array}$

$\begin{array}{lccccc}0 & 0 & 0 & 0 & 0 & 0 \\ 1 & 31 & 18 & 26 & 20 & 27 \\ 2 & 50 & 28 & 35 & 33 & 36 \\ 3 & 62 & 37 & 38 & 37 & 50 \\ 4 & 68 & 48 & 50 & 43 & 57 \\ 6 & 77 & 71 & -- & 51 & 69 \\ 9 & -- & -- & -- & 59 & 77 \\ 12 & -- & -- & -- & -- & -- \\ 15 & -- & -- & -- & -- & --\end{array}$

mean age:

(at exit / conditional on exit from consensual-union status)

1st decile at age:

1st quartile at:

median at age:

3rd quartile at:

1
1
2
6

$\begin{array}{cccc}1 & 1 & 1 & 1 \\ 2 & 1 & 2 & 1 \\ 5 & 4 & 6 & 3 \\ -- & -- & -- & 9\end{array}$

mean duration:

(of all episodes / truncated after 15 years)

age

$$
\text { Lithuania Poland }
$$

(1989-95) (1986-91)

$\begin{array}{lcc}0 & 0 & 0 \\ 1 & 29 & 3 \\ 2 & -- & 17 \\ 3 & -- & 24 \\ 4 & -- & -- \\ 6 & -- & -- \\ 9 & -- & -- \\ 12 & -- & -- \\ 15 & -- & --\end{array}$

mean age:

(at exit / conditional on exit from consensual-union status)

1st decile at age:

1st quartile at:

$1 \quad 2$

median at age:

3rd quartile at:

15

$\begin{array}{ll}1 & 5 \\ -- & --\end{array}$

mean duration:

(of all episodes / truncated after 15 years) 
Table 43: Cumulative percent ever again in a union, by time since union disruption, for children experiencing parental separation

\begin{tabular}{|c|c|c|c|c|c|}
\hline duration & $\begin{array}{l}\text { Sweden } \\
(1987-93)\end{array}$ & $\begin{array}{c}\text { Norway } \\
(1983-89)\end{array}$ & $\begin{array}{c}\text { Finland } \\
(1983-89)\end{array}$ & $\begin{array}{c}\text { France } \\
(1988-94)\end{array}$ & $\begin{array}{c}\text { USA } \\
(1989-95\end{array}$ \\
\hline 0 & 0 & 0 & 0 & 0 & 0 \\
\hline 1 & 11 & 17 & 15 & 10 & 21 \\
\hline 2 & 22 & 31 & 26 & 19 & 37 \\
\hline 3 & 32 & 41 & 31 & 23 & 47 \\
\hline 4 & 41 & 48 & 36 & 29 & 54 \\
\hline 6 & 51 & 57 & 45 & 35 & 67 \\
\hline 8 & 57 & 60 & 57 & 46 & 73 \\
\hline 10 & 62 & -- & 64 & 47 & 78 \\
\hline mean dur: & 3 & -- & 4 & 3 & 3 \\
\hline \multicolumn{6}{|c|}{ (at re-entry into union / conditional on union formation) } \\
\hline 1st decile at dur: & 1 & 1 & 1 & 1 & 1 \\
\hline 1st quartile at: & 3 & 2 & 2 & 4 & 2 \\
\hline median at dur: & 6 & 5 & 7 & -- & 4 \\
\hline 3rd quartile at: & -- & -- & -- & -- & 9 \\
\hline $\begin{array}{l}\text { mean duration: } \\
\text { (of all episodes / }\end{array}$ & $\begin{array}{c}6 \\
\text { after } 10 y\end{array}$ & ars) & 6 & 7 & 5 \\
\hline
\end{tabular}

\begin{tabular}{|c|c|c|c|c|c|}
\hline & & West & & & \\
\hline duration & $\begin{array}{c}\text { Austria } \\
(1990-96)\end{array}$ & $\begin{array}{c}\text { Germany } \\
(1986-92)\end{array}$ & $\begin{array}{c}\text { Flanders } \\
(1985-92)\end{array}$ & $\begin{array}{c}\text { Italy } \\
(1990-95)\end{array}$ & $\begin{array}{c}\text { Spain } \\
(1989-95)\end{array}$ \\
\hline
\end{tabular}

$\begin{array}{lccccc}0 & 0 & 0 & 0 & 0 & 0 \\ 1 & 18 & 8 & 21 & 2 & 5 \\ 2 & 25 & 24 & 29 & 2 & 23 \\ 3 & 30 & 29 & 37 & 8 & 25 \\ 4 & 36 & 39 & 42 & 13 & 29 \\ 6 & 47 & 50 & 54 & 28 & 37 \\ 8 & 51 & 56 & 64 & -- & 41 \\ 10 & 54 & -- & -- & -- & 47 \\ \text { mean dur: } & 3 & -- & -- & -- & 4 \\ \text { (at re-entry into union / conditional on union formation) } & & & \\ \text { 1st decile at dur: } & 1 & 2 & 1 & 4 & 2 \\ \text { 1st quartile at: } & 2 & 3 & 2 & 6 & 3 \\ \text { median at dur: } & 8 & 5 & 5 & -- & -- \\ \text { 3rd quartile at: } & -- & -- & -- & -- & - \\ \\ \text { mean duration: } \\ \text { (of all episodes / truncated after 10 years) }\end{array}$


Table 43: Cumulative percent ever again in a union, by time since union disruption, for children experiencing parental separation

\begin{tabular}{|c|c|c|c|c|c|}
\hline duration & $\begin{array}{c}\text { GDR } \\
(1984-89)\end{array}$ & $\begin{array}{c}\text { Hungary } \\
(1988-93)\end{array}$ & $\begin{array}{c}\text { Czech R } \\
(1992-97)\end{array}$ & $\begin{array}{c}\text { Slovenia } \\
(1989-95)\end{array}$ & $\begin{array}{c}\text { Latvia } \\
(1989-95)\end{array}$ \\
\hline 0 & 0 & 0 & 0 & 0 & 0 \\
\hline 1 & 28 & 15 & 32 & 14 & 16 \\
\hline 2 & 41 & 23 & 47 & 30 & 24 \\
\hline 3 & 55 & 39 & 61 & 36 & 32 \\
\hline 4 & 58 & 47 & 66 & 50 & 38 \\
\hline 6 & 65 & 57 & 71 & 62 & 43 \\
\hline 8 & 68 & 68 & 75 & 69 & 51 \\
\hline 10 & -- & 68 & 77 & 71 & 60 \\
\hline mean dur: & -- & 3 & 2 & 3 & 4 \\
\hline \multicolumn{6}{|c|}{ (at re-entry into union / conditional on union formation) } \\
\hline 1st decile at dur: & 1 & 1 & 1 & 1 & 1 \\
\hline 1st quartile at: & 1 & 3 & 1 & 2 & 3 \\
\hline median at dur: & 3 & 5 & 3 & 4 & 8 \\
\hline 3rd quartile at: & -- & -- & 8 & -- & -- \\
\hline $\begin{array}{l}\text { mean duration: } \\
\text { (of all episodes / }\end{array}$ & after 1 & 5 & 4 & 5 & 6 \\
\hline
\end{tabular}

$\begin{array}{llc}\text { duration } & \text { Lithuania } & \text { Poland } \\ (1989-95) & (1986-91)\end{array}$

$\begin{array}{lcc}0 & 0 & 0 \\ 1 & 9 & 7 \\ 2 & 18 & 15 \\ 3 & 25 & 19 \\ 4 & 30 & 20 \\ 6 & 43 & 25 \\ 8 & 52 & 38 \\ 10 & 57 & 38\end{array}$

mean dur: $4 \quad 4$

(at re-entry into union / conditional on union formation)

1st decile at dur: $\quad 2 \quad 2$

1st quartile at: $\quad 3 \quad 5$

median at dur: $\quad 8$

3rd quartile at: $\quad$-- $\quad--$

mean duration: $\quad 7 \quad 8$

(of all episodes / truncated after 10 years) 
Table 44: Cumulative percent ever in marriage, by time since union disruption, for children experiencing parental separation

$\begin{array}{lccccc}\text { duration } & \text { Sweden } & \text { Norway } & \text { Finland } & \text { France } & \text { USA } \\ & (1987-93) & (1983-89) & (1983-89) & (1988-94) & (1989-95)\end{array}$

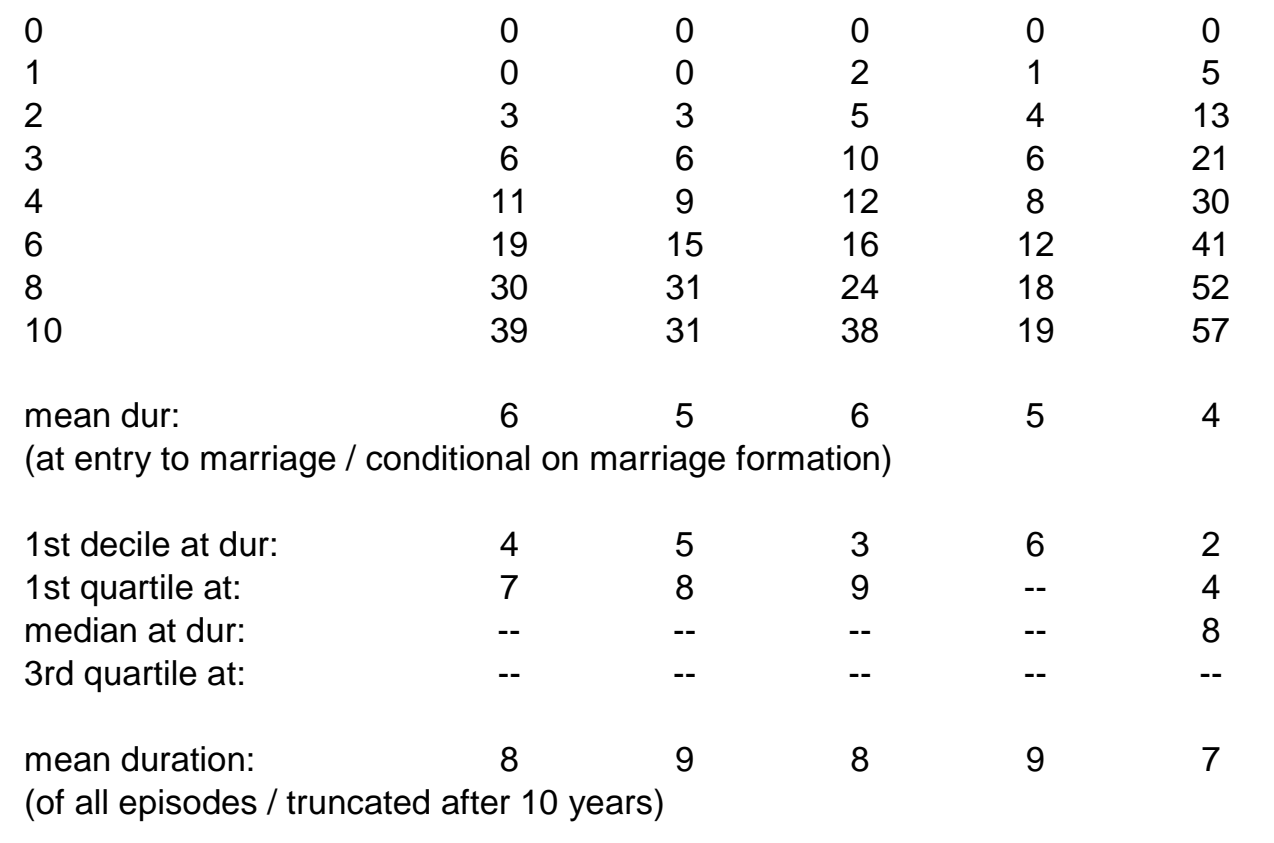

$\begin{array}{ccccc}\text { duration } & \text { West } & & \\ \text { Austria } & \text { Germany } & \text { Flanders } & \text { Italy } & \text { Spain } \\ (1990-96) & (1986-92) & (1985-92) & (1990-95) & (1989-95)\end{array}$

\begin{tabular}{|c|c|c|c|c|c|}
\hline 0 & 0 & 0 & 0 & 0 & 0 \\
\hline 1 & 3 & 0 & 1 & 0 & 0 \\
\hline 2 & 7 & 4 & 2 & 0 & 1 \\
\hline 3 & 9 & 12 & 11 & 0 & 2 \\
\hline 4 & 15 & 20 & 17 & 0 & 4 \\
\hline 6 & 21 & 36 & 25 & 6 & 10 \\
\hline 8 & 23 & 42 & 30 & -- & 10 \\
\hline 10 & 30 & -- & 35 & -- & 12 \\
\hline mean dur: & 5 & -- & 5 & -- & 5 \\
\hline \multicolumn{6}{|c|}{ (at entry to marriage / conditional on marriage formation) } \\
\hline 1st decile at dur: & 4 & 3 & 3 & -- & 6 \\
\hline 1st quartile at: & 9 & 5 & 6 & -- & -- \\
\hline median at dur: & -- & -- & -- & -- & -- \\
\hline 3rd quartile at: & -- & -- & -- & -- & -- \\
\hline $\begin{array}{l}\text { mean duration: } \\
\text { (of all episodes / }\end{array}$ & 8 & -- & 8 & -- & 9 \\
\hline
\end{tabular}


Table 44: Cumulative percent ever in marriage, by time since union disruption, for children experiencing parental separation
duration
GDR Hungary Czech R Slovenia Latvia (1984-89) (1988-93) (1992-97) (1989-95) $\quad$ (1989-95)

$\begin{array}{lccccc}0 & 0 & 0 & 0 & 0 & 0 \\ 1 & 7 & 3 & 2 & 0 & 5 \\ 2 & 12 & 5 & 15 & 0 & 7 \\ 3 & 25 & 11 & 26 & 6 & 11 \\ 4 & 28 & 18 & 30 & 13 & 17 \\ 6 & 42 & 28 & 36 & 21 & 20 \\ 8 & 51 & 34 & 45 & 26 & 24 \\ 10 & 54 & 38 & 52 & 31 & 29 \\ \text { mean dur: } & 4 & 5 & 4 & 5 & 4 \\ \text { (at entry to marriage / conditional on marriage formation) } & & \\ \text { 1st decile at dur: } & 2 & 3 & 2 & 4 & 3 \\ \text { 1st quartile at: } & 3 & 6 & 3 & 7 & 9 \\ \text { median at dur: } & 8 & -- & 10 & -- & -- \\ \text { 3rd quartile at: } & -- & -- & -- & -- & -- \\ & & & & & \\ \text { mean duration: } & 7 & 8 & 7 & 9 & 8\end{array}$

(of all episodes / truncated after 10 years)

$\begin{array}{ccc}\text { duration } & \text { Lithuania } & \text { Poland } \\ (1989-95) & (1986-91)\end{array}$

\begin{tabular}{|c|c|c|}
\hline 0 & 0 & 0 \\
\hline 1 & 3 & 0 \\
\hline 2 & 8 & 5 \\
\hline 3 & 14 & 8 \\
\hline 4 & 17 & 8 \\
\hline 6 & 29 & 13 \\
\hline 8 & 35 & 23 \\
\hline 10 & 37 & 23 \\
\hline mean dur: & 4 & 5 \\
\hline (at entry to marriac & onal & riage formation) \\
\hline 1st decile at dur: & 3 & 5 \\
\hline 1st quartile at: & 6 & -- \\
\hline median at dur: & -- & -- \\
\hline 3rd quartile at: & -- & -- \\
\hline $\begin{array}{l}\text { mean duration: } \\
\text { (of all episodes / tr }\end{array}$ & 8 & 9 \\
\hline
\end{tabular}


Table 45: Percent of time spent in different family types at ages $0-14$ years

$\begin{array}{ccccc}\text { Sweden } & \text { Norway } & \text { Finland } & \text { France } & \text { USA } \\ (1987-93) & (1983-89) & (1983-89) & (1988-94) & (1989-95)\end{array}$

time with lone mother, from birth time with lone mother, after disruption time after leaving mother $==>$ time with lone or no mother time with both parents in consensual union time with both parents in marriage $==>$ time with both parents time in step union, with mother

$\begin{array}{cccccccccc}2 & & 2 & & 1 & & 4 & & 7 & \\ 9 & & 5 & & 6 & & 7 & & 13 & \\ 2 & & 0 & & 1 & & 0 & & 2 & \\ & 12 & & 7 & & 8 & & 11 & & 22 \\ 17 & & 5 & & 4 & & 8 & & 4 & \\ 64 & & 84 & & 84 & & 78 & 64 & \\ & 81 & & 89 & & 88 & & 86 & & 67 \\ 6 & & 4 & & 4 & & 3 & & 10 & \end{array}$

time with lone mother, from birth time with lone mother, after disruption time after leaving mother $==>$ time with lone or no mother time with both parents in consensual union time with both parents in marriage $==>$ time with both parents time in step union, with mother

\begin{tabular}{|c|c|c|c|c|}
\hline & Vve & & & \\
\hline $\begin{array}{c}\text { Austria } \\
(1990-96)\end{array}$ & $\begin{array}{c}\text { Germany } \\
(1986-92)\end{array}$ & $\begin{array}{c}\text { Flanders } \\
(1985-92)\end{array}$ & $\begin{array}{c}\text { Italy } \\
(1990-95)\end{array}$ & $\begin{array}{c}\text { Spain } \\
(1989-95)\end{array}$ \\
\hline
\end{tabular}

$\begin{array}{ccccccccccc}4 & & 2 & & 0 & & 0 & & 1 & \\ 8 & & 8 & & 4 & & 2 & & 3 & \\ 1 & & 1 & & 0 & & 0 & & 0 & \\ & 12 & & 12 & & 5 & & 3 & & 4 \\ 6 & & 5 & & 1 & & 1 & & 1 & \\ 79 & & 78 & & 91 & & 95 & & 93 & \\ & & & & & & & & & & \\ 4 & & & 83 & & 92 & & 97 & & 94 \\ 4 & & 5 & & 3 & & 0 & & 1 & \end{array}$


Table 45: Percent of time spent in different family types at ages $0-14$ years

\begin{tabular}{|c|c|c|c|c|}
\hline $\begin{array}{c}\text { GDR } \\
(1984-89)\end{array}$ & $\begin{array}{c}\text { Hungary } \\
(1988-93)\end{array}$ & $\begin{array}{c}\text { Czech R } \\
(1992-97)\end{array}$ & $\begin{array}{c}\text { Slovenia } \\
(1989-95)\end{array}$ & $\begin{array}{c}\text { Latvia } \\
(1989-95)\end{array}$ \\
\hline 5 & 1 & 1 & 2 & 3 \\
\hline 8 & 6 & 6 & 3 & 11 \\
\hline 1 & 0 & 0 & 1 & 0 \\
\hline 13 & 8 & 8 & 6 & 15 \\
\hline 6 & 2 & 2 & 6 & 3 \\
\hline 73 & 86 & 84 & 86 & 75 \\
\hline $8 \quad 79$ & $5^{87}$ & $7^{85}$ & $2^{92}$ & 79 \\
\hline
\end{tabular}

time with lone mother, from birth time with lone mother, after disruption time after leaving mother $==>$ time with lone or no mother time with both parents in consensual union time with both parents in marriage $==>$ time with both parents time in step union, with mother

\section{Lithuania Poland}

(1989-95) (1986-91)

time with lone mother, from birth time with lone mother, after disruption time after leaving mother $==>$ time with lone or no mother

$\begin{array}{ccccc}2 & & 5 & \\ 8 & & 3 & \\ 0 & & 0 & \\ & 10 & & 9 \\ 1 & & 1 & \\ 85 & & 90 & \\ & 86 & & 90 \\ 3 & & & 1 & \end{array}$

time with both parents in consensual union time with both parents in marriage $==>$ time with both parents time in step union, with mother 